\title{
Simultaneous Analysis of Ion and Electron Heat Transport by Power Modulation in JET
}

\author{
F. Ryter ${ }^{1}$, C. Angioni ${ }^{1}$, C. Giroud ${ }^{2}$, A.G. Peeters ${ }^{3}$, T. Biewer ${ }^{4}$, \\ R. Bilato ${ }^{1}$, E. Joffrin ${ }^{5}$, T. Johnson ${ }^{6}$, H. Leggate $^{2}$, \\ E. Lerche ${ }^{7}$, G. Madison ${ }^{2}$, P. Mantica ${ }^{8}$, D. Van Eester ${ }^{7}$,
} I. Voitsekhovitch ${ }^{2}$ and JET Contributors $\ddagger$ JET-EFDA, Culham Science Centre, OX14 3DB, Abingdon, UK

${ }^{1}$ Max-Planck-Institut für Plasmaphysik, EURATOM Association, D-85748 Garching ${ }^{2}$ Culham Science Centre, EURATOM/UKAEA Association, OX14 3DB, Abingdon,UK ${ }^{3}$ Centre for Fusion Space and Astrophysics, University of Warwick, Coventry 7AL, UK ${ }^{4}$ Fusion Energy Division, Oak Ridge National Lab. P.O. Box 2008 MS 6169 Oak Ridge USA ${ }^{5}$ JET-EFDA-CSU, Culham Science Centre, OX14 3DB, Abingdon, UK ${ }^{6}$ EURATOM-VR Association, Fusion Plasma Physic, EES, KTH 10044 Stockholm, Sweden ${ }^{7}$ LPP-ERM/KMS, Association Euratom-Belgian State, TEC, B-1000 Brussels, Belgium ${ }^{8}$ Istituto di Fisica del Plasma, EURATOM/ENEA-CNR Association, 20125, Milano, Italy E-mail: ryter@ipp.mpg • de

\begin{abstract}
Heating power modulation experiments using ICRH in the ${ }^{3} \mathrm{He}$ minority scheme have been performed in the JET tokamak to investigate heat transport properties. This RF scheme provides a dominant localised ion heating, but also some electron heating, and therefore both ion and electron heat channels were modulated. This allows to carry out a simultaneous transport analysis of ion and electron heat transport channels, including transient transport phenomena. This also provides an experimental assessment of the ICRH heat sources of the ${ }^{3} \mathrm{He}$ scheme. The modulation approach, so far widely used for electron transport studies, has been validated for ion heat transport in these experiments and yields results on stiffness and threshold of the ITG-driven ion heat transport. The results for the electron channel demonstrate the importance of the ITG-driven, off-diagonal, contribution to electron heat transport in plasmas with significant ion heating.
\end{abstract}

\$ See the appendix of F. Romanelli et al., Proceedings of the 23rd IAEA Fusion Energy Conference 2010, Daejeon, Korea 


\section{Introduction}

In fusion plasmas, turbulent phenomena driven by the Ion Temperature Gradient and Trapped Electron Mode instabilities in general dominate ion and electron heat transport. The ITG and TEM modes are unstable above respective thresholds in normalised temperature gradients, $-R \nabla T / T=R / L_{T}$, leading to an increase of transport above the threshold $R / L_{T, \text { crit }}$, see for instance [1] for ITG and [2] for TEM. In addition, transport driven by micro-turbulence can be assumed, in first approximation, to exhibit a gyro-Bohm behaviour, such that transport is proportional to $T^{3 / 2}$. The rate with which transport increases above the threshold is called here "stiffness factor" and characterised by a coefficient $\chi_{s}$ which will be defined below. This leads for the heat diffusion to the assumption $\chi \propto \chi_{S} T^{3 / 2}\left(R / L_{T}-R / L_{T, c r i t}\right)$, as described in detail in section 4.2. The observed resilience, often called profile stiffness, of the temperature profile shape to changes in heating power deposition, [3, 4, 5, 6, 7, 8, 9, 10], can be explained by these properties [11]. For the electrons, the existence of both threshold and stiffness have been directly evidenced experimentally in several devices $[12,13,14,15]$, but these properties have been only recently investigated for ions $[16,17]$.

"Perturbative transport" experiments, in which temperature perturbations induced by modulating the heating power are analysed, yield the stiffness properties. This method has been extensively used to investigate electron heat transport properties, see e.g. $[18,19,20$, $14,21,11,22]$, but not applied to the ions so far, essentially because a method for localised ion heating, together with a $T_{i}$ measurement with sufficient time resolution were not available simultaneously in the same device. As reported in [16], this has been made possible rather recently at JET by the Ion Cyclotron Resonant Heating in the ${ }^{3} \mathrm{He}$ minority scheme and thanks to an upgrade of the $T_{i}$ measurement by Charge Exchange Recombination Spectroscopy (CXRS).

The main goal of the work presented here was to modulate the ion temperature, assess the validity of the method under these conditions and deduce experimentally the properties of ion heat transport. As a fraction of the electron heating power was also modulated, electron heat transport is also investigated. This allows a comprehensive and simultaneous investigation of both ion and electron heat transport by perturbative methods.

The experiments are described in Sect. 2. Section 3 is dedicated to the experimental analysis of the modulated ion temperature data. In section 4, assumptions and requirements for transport modelling are described, whereas the results for the ion and electron channels are 
presented respectively in sections 5 and 6 . Conclusions are drawn in the last section.

\section{Experiments and method of analysis}

\subsection{Experimental conditions}

The experiments presented here have been carried out in the JET tokamak, with major radius $R \approx 3 \mathrm{~m}$ and and minor radius $a \approx 1 \mathrm{~m}$, at a rather high edge safety factor value, $q_{95} \approx 6$ which allow to avoid sawteeth as $q$ can be kept above unity in the centre. The absence of sawteeth ensures good conditions for a reliable analysis of modulation data. They were run in deuterium L-modes at a density of about $3 \times 10^{19} \mathrm{~m}^{-3}$, heated by deuterium Neutral Beam Injection as background heating and Ion Cyclotron Resonance Heating using the ${ }^{3} \mathrm{He}$ minority scheme for the power modulation part. The RF power was modulated at $f_{\text {mod }}$ with a 50/50 duty-cycle and a modulation amplitude of about $\pm 40 \%$. JET offers a unique opportunity for localised ion and electron heating by ICRH in the ${ }^{3} \mathrm{He}$ minority scheme, whose power deposition properties depend on the ${ }^{3} \mathrm{He}$ concentration, $\left[{ }^{3} \mathrm{He}\right]$, as described in [23]. The ion heating is provided by the ${ }^{3} \mathrm{He}$ ICRH-accelerated ions, with rather low energy, which deliver a dominant fraction of their energy to the plasma as ion heating. Due to the higher ion mass, this contribution is significantly larger than in the hydrogen minority scheme. The fraction of ion heating increases with $\left[{ }^{3} \mathrm{He}\right]$ to reach a maximum at $\left[{ }^{3} \mathrm{He}\right] \approx 8 \%$. If $\left[{ }^{3} \mathrm{He}\right]$ is further increased, the power absorbed by the ${ }^{3} \mathrm{He}$ minority decreases and the ion heating fraction as well, while the direct electron heating fraction increases. On each side of its maximum, the fraction of ion heating is lower by about $20 \%$ for $\left[{ }^{3} \mathrm{He}\right] \approx 6 \%$ and $\left[{ }^{3} \mathrm{He}\right] \approx 12 \%$. The power deposition is calculated by the PION code [24] which yields the time-dependent ICRF heat sources for our transport analyses, taking modulation into account. We also get ICRH power deposition profiles from the SELFO code, [25] and references therein. These are not time-dependent, but provide a more accurate modelling of the collisional energy transfer of the RF-accelerated fast ions to the electron and ion channels. In addition, electron heating by mode conversion to Bernstein and ion cyclotron waves also occurs, with a maximum for $\left[{ }^{3} \mathrm{He}\right] \approx 30 \%,[26]$. This component can be calculated by the full wave code TORIC, [27]. As usual in such experiments at JET, the ${ }^{3} \mathrm{He}$ concentration was measured in the edge region and feed-back controlled, as described in [23]. Only one profile of $\left[{ }^{3} \mathrm{He}\right]$, with rather large uncertainties, is available. 
The measurement of the ion temperature, which is the basis of the investigations presented here, is provided by the CXRS diagnostic. The experiments have been carried out in three distinct series of discharges, in 2002, 2003 and 2006. In the 2002 and 2003 campaigns, the time resolution of the $T_{i}$ measurement was $50 \mathrm{~ms}$ which limited the maximum modulation frequency to about $4 \mathrm{~Hz}$. However, the 2002 discharges demonstrated that it was possible and meaningful to address ion perturbative transport with this scenario. In the 2003 series, we varied the NBI heating to investigate the possible influence of $T_{i}$. However, the results of these two series required validation of the physics interpretation by experiments at higher modulation frequencies. This was finally possible in the 2006 campaign for which the CXRS diagnostic had been upgraded, providing measurements with a time resolution of 10 ms $[28,29]$. We obtained modulation data with good signal-to-noise ratio from an ICRH modulation frequency scan $4 \leq f_{\text {mod }} \leq 20 \mathrm{~Hz}$. Data from the edge CXRS diagnostics are not available. As discussed below, not only the modulation of $T_{i}$ but also that of $T_{e}$ are analysed in these experiments. The $T_{e}$ measurement is provided by the Electron Cyclotron Emission radiometer diagnostic which has a much higher spatial and temporal resolution than the CXRS diagnostic.

In Fig. 1, a representative discharge from the 2006 campaign with $8 \mathrm{~Hz}$ ICRH power modulation is illustrated. The ICRH power is modulated with an amplitude depth of about $\pm 40 \%$ around the time-averaged power, with peak power of about $4 \mathrm{MW}$, and is added to the back-ground of about $5 \mathrm{MW}$ of constant NBI power. The ICRH power is deposited close to the plasma centre, as shown in a later section. The induced ion temperature modulation is clearly visible, as indicated by a few time traces at different radial positions, defined by the normalised toroidal flux radius $\rho$, box a. The electron temperature is also significantly modulated, as seen in box b. Both temperatures also exhibit slow variations in which the maxima correspond to somewhat more peaked profiles. These slow oscillations in $T_{i}$ and $T_{e}$ are not absolutely in phase, but seem to be strongly correlated. They do not occur in all discharges but seem to appear for values of the ${ }^{3} \mathrm{He}$ concentration above 6-10\%. Therefore, one may conjecture that they are linked to variations of the ${ }^{3} \mathrm{He}$ concentration profile leading to changes of the electron and ion heating profiles. The slow oscillations only weakly affect the results presented later, as can be assessed by analysing different time intervals. Finally, as shown in box c, a modulation of the edge channel of the line-integrated density is induced by the ICRF power, whereas the core channel plotted, in panel d, exhibits almost no modulation. 


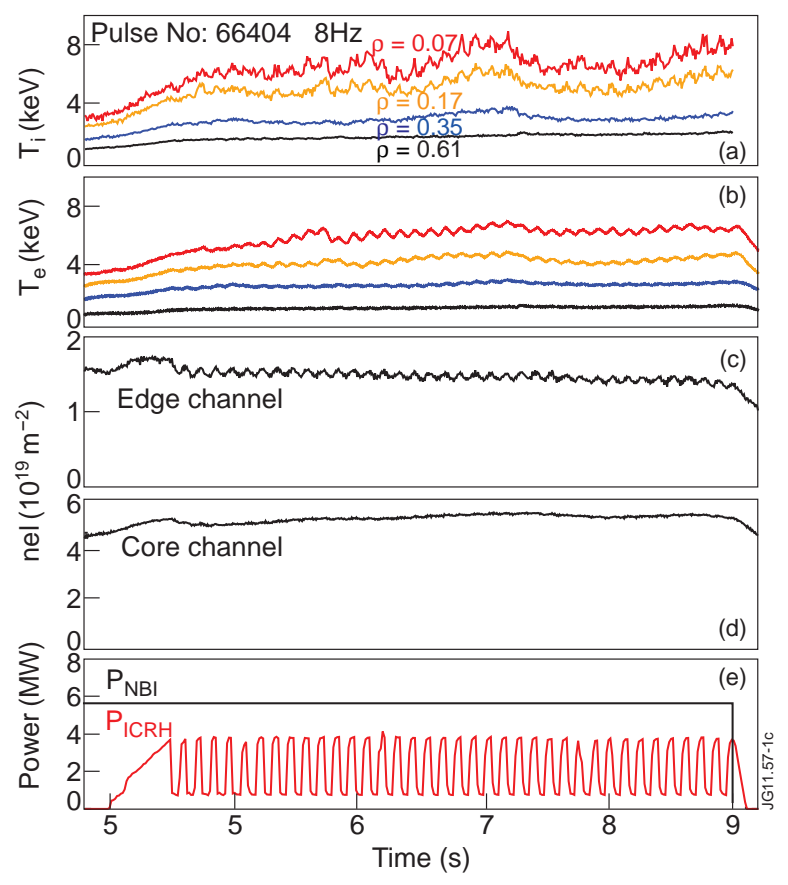

Figure 1. Time evolution of a discharge with $8 \mathrm{~Hz}$ modulation. The ICRH power modulation is indicated in the panel e, together with the constant NBI power of about $5 \mathrm{MW}$. Boxes $a$ and $b$ illustrate the $T_{i}$ and $T_{e}$ signals at the 4 radial positions indicated in box a. The line of sight of the interferometer in the edge region plotted in panel $c$ exhibits the edge density modulation discussed in the text, whereas the core channel plotted in box $d$ is not modulated.

This indicates that this density modulation is restricted to the edge region of the plasma. This density modulation is indeed restricted to the edge region and not observed in the centre. This is in agreement with the assumption that it is excited at the very edge and is strongly damped as it propagates inwards. The Fourier analysis of the density modulation indicates that its phase delay with respect to the ICRH input power increases from about 35 degrees at $4 \mathrm{~Hz}$ up to 80 degrees at $20 \mathrm{~Hz}$. This density modulation is roughly proportional to the ICRH modulation amplitude, at fixed value of $f_{\text {mod }}$ and strongly decreases with increasing $f_{\text {mod }}$. The influence of this modulation on the analysis will be discussed later.

\subsection{Experimental Method of analysis}

To investigate heat transport, perturbative experiments have been, so far, only widely applied to the electron channel, but the method can also be applied to ions in the same way [16]. In 
this approach, the propagation of the excited heat pulses is determined by the slope of the heat flux with respect to the temperature gradient, yielding the heat pulse diffusivity $[30,18]$ :

$$
\chi_{j}^{H P}=-\frac{\partial q_{j}}{\partial\left(n_{j} \nabla T_{j}\right)}=\chi_{j}^{P B}+\frac{\partial \chi_{j}}{\partial \nabla T_{j}} \nabla T_{j}
$$

where the subscript $j$ denotes $e$ or $i$ (electron or ion), $q$ heat flux and $n$ density, whereas $\chi_{j}^{P B}$ is the usual heat diffusivity from power balance. In the experiments, $\chi_{j}^{H P}$ is derived from the amplitude $(A)$ and phase $(\varphi)$ profiles provided by the Fourier transform of the temperature data and interpreted in slab geometry $[18,31]$, to which corrections for cylindrical geometry and density gradient are usually added [32]. This method yields 2 estimates for the heat pulse diffusivity,

$$
\chi_{\text {exp }}^{\text {amp }}=\frac{3 \omega_{\text {mod }}}{4\left(A^{\prime} / A\right)^{2}} \quad \chi_{\text {exp }}^{p h i}=\frac{3 \omega_{\text {mod }}}{4 \varphi^{\prime 2}}
$$

where $\omega$ is the modulation pulsation, $A^{\prime}$ and $\varphi^{\prime}$ mean radial derivatives. Due to damping processes, which affect amplitude and phase profiles, these 2 quantities differ at low modulation frequencies such that $\chi_{j}^{p h i} \geq \chi_{j}^{A m p}$, but converge asymptotically towards $\chi_{j}^{H P}$ at high modulation frequencies for which damping becomes negligible. However, in the geometric mean, $\sqrt{\chi_{j}^{A m p} \chi_{j}^{p h i}}$, the damping effects mathematically cancel and, at any frequency, this quantity can be considered as a good experimental estimator of the actual value of $\chi_{j}^{H P}$. Note that $\chi_{j}^{A m p}, \chi_{j}^{p h i}$ and $\chi_{j}^{H P}$ depend on the square of gradients and are therefore quite sensitive to the measured amplitude and phase profiles.

In the present work, this method is applied to ion and electron modulated data. In this section, we focus on the ion data. As example, the method is illustrated in Fig. 2 for modulation data at $8 \mathrm{~Hz}$, corresponding to the discharge illustrated in Fig. 1. As usually done in such studies, we plot the profile of the natural logarithm of the amplitude of the $T_{i}$ modulation because its slope directly reflects $\chi_{i}^{A m p}$. The phase delay is calculated with respect to the phase of the injected modulated ICRH power. The error bars indicated in the plots are deduced from the signal-to-noise ratio for each radial data point. The noise level is estimated from the data at frequencies in a narrow band on each side of the modulation frequency which is, as being imposed from outside, a very narrow peak in the frequency spectrum. Figure 2 indicates that the experimental uncertainties are small enough to determine the heat pulse propagation with sufficient accuracy for physics interpretation.

The heat pulse analysis is carried out in the plasma confinement zone, around $R=3.4 \mathrm{~m}$ and we plot here examples of the fits used to determine $\chi_{i}^{A m p}$ and $\chi_{i}^{p h i}$ whose corresponding values 

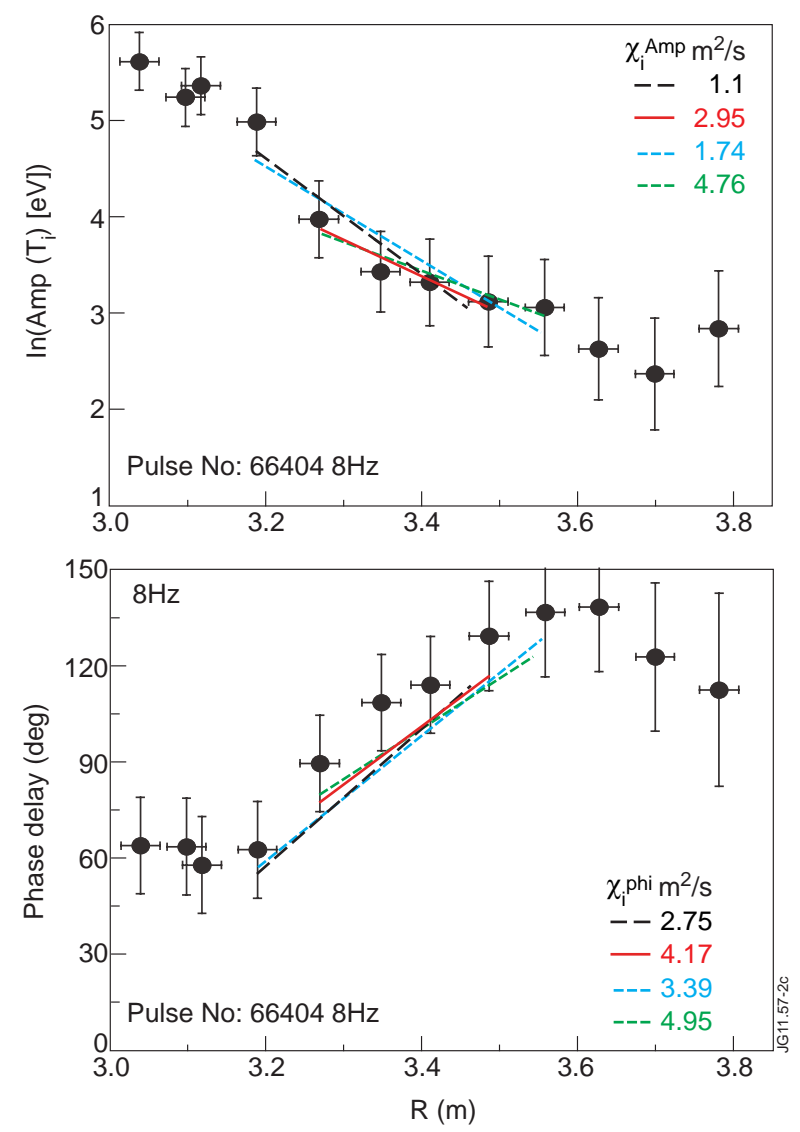

Figure 2. Profiles of amplitude and phase delay of modulation data at $8 \mathrm{~Hz}$. As examples, fits for $\chi_{i}^{A m p}$ and $\chi_{i}^{\text {phi }}$ are also drawn, with corresponding values.

are also indicated in Fig. 2. Including in the fits more or less data points, at larger or smaller radii, provides an estimate of the experimental uncertainties which are used in the results presented later. It should be reminded that this analysis is only valid in plasma regions free of modulated power. In this case, transport is reflected by the gradient of the amplitude and phase profiles (Eqs. 2): low gradients correspond to high transport and/or high stiffness, steep gradients to low transport and stiffness. It is also well-known that these gradients become steeper when the modulation frequency is increased. Finally, it should be emphasised that the interpretation of $\chi_{i}^{A m p}$ and $\chi_{i}^{p h i}$ is local and reflects transport properties in the radial region over which the fits are made.

In the region where the modulated power is deposited, amplitude and phase behave as follows, see e.g. [33]. The amplitude, in this region, decreases if modulation frequency or transport are increased. In the region of the power deposition, the phase delay with respect to the ICRF power modulation is the sum of two contributions: the reaction of the temperature to 
power modulation as determined by transport properties and an additional delay due to the collisional power transfer of the RF-accelerated ions. The phase delay caused by the former effect increases with increasing modulation frequency, but decreases if transport increases, [33]. The latter is provided by the calculations of the ICRF power absorption described later. In Fig. 2, the amplitude exhibits an increase at the plasma edge $(R>3.7 \mathrm{~m})$, whereas the phase delay decreases in the edge region. This is attributed to the density modulation mentioned above, which induces an edge temperature modulation in $T_{i}$ which propagates towards the center. This parasitic modulation interferes with the $T_{i}$ modulation excited in the centre by the heating modulation and whose propagation has to be analysed to deduce $\chi_{i}^{H P}$. It is therefore essential to assess the influence of the spurious $T_{i}$ modulation excited at the edge on the transport analysis.

For this purpose, we calculate the interference of two heat waves excited in the core and at the edge and propagating respectively outwards and inwards with a simple model in cylindrical geometry. The propagation velocity of these two singles waves are adjusted such that amplitude and phase profiles of the combined wave match the experimental ones simultaneously. The results for 2 frequencies are shown in Fig. 3 which shows the amplitude and phase profiles of the single wave, as well as those of their combination which has to be compared to the experimental data. The good match with the experimental profiles exhibited by these plots is only achieved if the propagation of the perturbation excited at the edge is significantly slower than that of the $T_{i}$ perturbation induced in the centre and propagating outwards. The difference in propagation velocity is the only possibility to produce the respective minimum and maximum of amplitude and phase at $R \approx 3.7 \mathrm{~m}$ and not at mid-radius. This is also a prerequisite to reproduce the different slopes on either side of the extrema. This is in agreement with the fact that particle diffusion is known to be lower than heat diffusion. This also suggests that the perturbation of $T_{i}$ caused by the density modulation is not a $T_{i}$ cold heat wave propagating inwards, but rather a direct effect of the density perturbation. Independently of the physics assumption for the parasitic edge modulation, the model clearly indicates that the modulation induced in the centre dominates both amplitude and phase profiles in the region $\rho \leq 0.7$, which corresponds to about $R \leq 3.6 \mathrm{~m}$. Therefore, the results deduced from heat pulse propagation, for which we rely on the modulation induced in the centre, are valid at least for $R \leq 3.6 \mathrm{~m}$ where this wave dominates. 

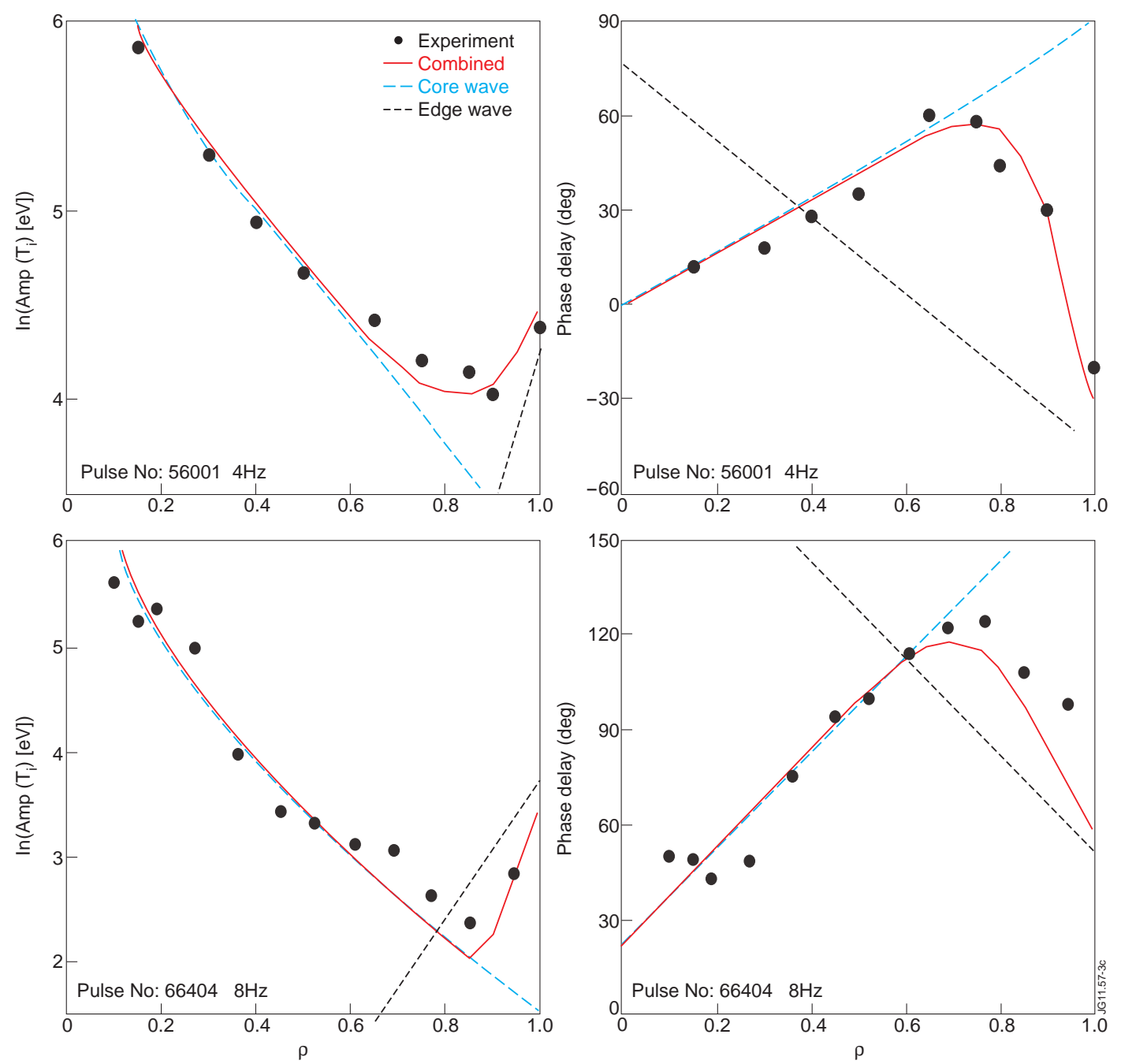

Figure 3. Profiles of amplitude and phase delay for for two $T_{i}$ heat waves launched from the center and from the edge. Two examples are shown with modulation frequencies of $4 \mathrm{HZ}$ and $8 \mathrm{~Hz}$. The points are the experimental data. The lines are yielded by the model described in the text.

\section{Results of the modulated $T_{i}$ data}

The analysis of the $T_{e}$ modulation by Fourier transform is a widely used method in transient transport studies for the electron heat channel. The high time resolution of the ECE diagnostic does not limit the modulation frequency and its high sensitivity provides in general data with a very good signal-to-noise ratio.

The situation for $T_{i}$ is different: the time resolution of the CXRS data can limit the highest modulation frequency. This was the case for the 2002 and 2003 series with the $50 \mathrm{~ms}$ time resolution. The $10 \mathrm{~ms}$ exposure time available for 2006 provided much better conditions 
and room for optimising the signal-to-noise ratio. The best $\mathrm{S} / \mathrm{N}$ is a compromise between the integration time on which the $T_{i}$ analysis is carried out and the number of measurement time points available for the Fourier transform. In our experiments, several CXRS exposure frames with $10 \mathrm{~ms}$ integration time can be grouped prior to the $T_{i}$ analysis. At low modulation frequency, up to 5 frames can be grouped without affecting the phase. This demonstrates the validity of the 2002 and 2003 data with 50ms resolution at $4 \mathrm{~Hz}$. With 10ms exposure time, the quality of the modulated $T_{i}$ data is good under our experimental conditions up to $20 \mathrm{~Hz}$ and the best $\mathrm{S} / \mathrm{N}$ is obtained when grouping at most 2 or 3 frames, depending on the cases. Thus, for 2006, the improvement of the CXRS system and the possibility of grouping the frames provides an excellent set of data to investigate the ion heat pulse propagation in the frequency scan discussed below.

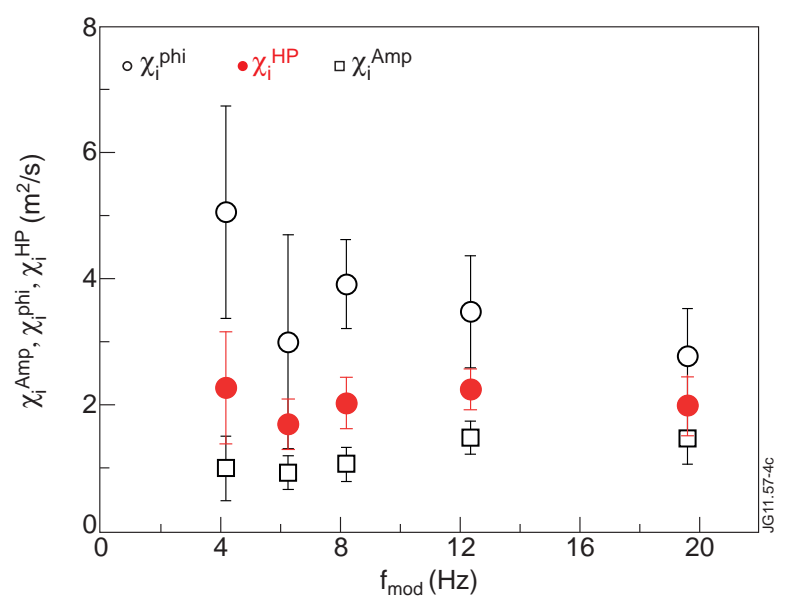

Figure 4. Experimental results from perturbative transport analysed at $R=3.4 \mathrm{~m}$ for the frequency scan. Dependence of $\chi_{i}^{\text {phi }}, \chi_{i}^{\text {Amp }}$ and $\chi_{i}^{H P}$ versus $f_{\text {mod }}$.

The perturbative transport results of the frequency scan are represented by $\chi_{i}^{p h i}, \chi_{i}^{A m p}$ and $\chi_{i}^{H P}$ versus $f_{\text {mod }}$, analysed at about mid-radius $(R \approx 3.4 \mathrm{~m})$ and plotted in Fig. 4 . In this figure, the values are the mean of the values yielded by all the realistic fits which can be made using the available data for each frequency, whereas the error bars are provided by the corresponding standard deviation. As expected, $\chi_{i}^{p h i}$ is larger than $\chi_{i}^{A m p}$ and these two quantities converge towards $\chi_{i}^{H P}$ as $f_{\text {mod }}$ increases. The value of $\chi_{i}^{H P}$, close to $2 \mathrm{~m}^{2} / \mathrm{s}$, does not depend on $f_{\text {mod }}$. This frequency scan indicates that ion perturbative transport exhibits the expected basic properties. Therefore, we conclude that this method can be used to investigate the properties of ion heat transport. This frequency scan also indicates that the data at $f_{\text {mod }}=$ 
$4 \mathrm{~Hz}$, obtained in the 2002 and 2003 series are valid, within the restrictions linked to the low frequency. In particular, $\chi_{i}^{H P}$ in the outer part of the plasma cannot be deduced due to the influence of the edge boundary condition. The analysis of $\chi_{i}^{H P}$ at this modulation frequency is possible at all in these discharges because the ion stiffness is low enough to allow physics interpretation, as shown below and in [17]. Indeed, measuring higher stiffness would require higher modulation frequency to capture the propagation of the heat pulses correctly.

As reported in [17], in discharges with the same controlled plasma parameters and also heated with NBI and ICRH heating at comparable levels, the $T_{i}$ profiles become more peaked when the NBI power is increased, which is attributed to a decrease of core stiffness with increasing toroidal rotation, while this does not happen in the outer plasma region. The increase of $R / L_{T_{i}}$ in the central plasma with heating power and toroidal rotation driven by NBI is also observed in our discharges, as revealed by the analysis of the power scan of the 2002 and 2003 series. For comparison, the 2006 data are also included. This is illustrated in Fig. 5 where $R_{L} / T_{i}$ in the plasmas inner part $(R \approx 3.3 \mathrm{~m})$ and outer region $(R \approx 3.6 \mathrm{~m})$ are plotted. Indeed the former increases by about a factor of two within the power range whereas the latter is almost constant within the error bars.

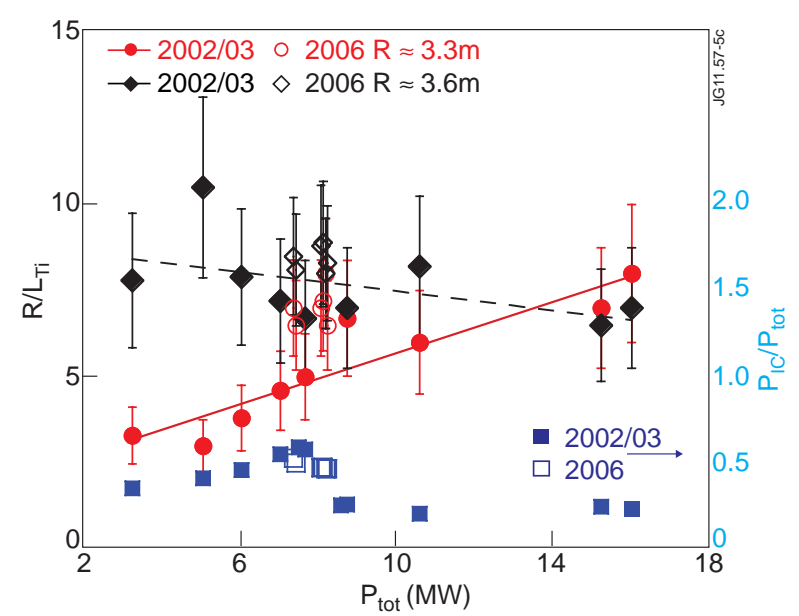

Figure 5. Experimental results from the 2002, 2003 and 2006 series: $R / L_{T_{i}}$ in the inner part and outer part of the plasma around $R \approx 3.3 \mathrm{~m}$ and $R \approx 3.6 \mathrm{~m}$ respectively. The lines are linear least square fits to the 2002 and 2003 data. The ratio of ICRF power to the total heating power is also given.

The dependence as a function of toroidal angular rotation taken at position of the $R / L_{T_{i}}$ analysis exhibits the same trends, as shown in Fig. 6 . 


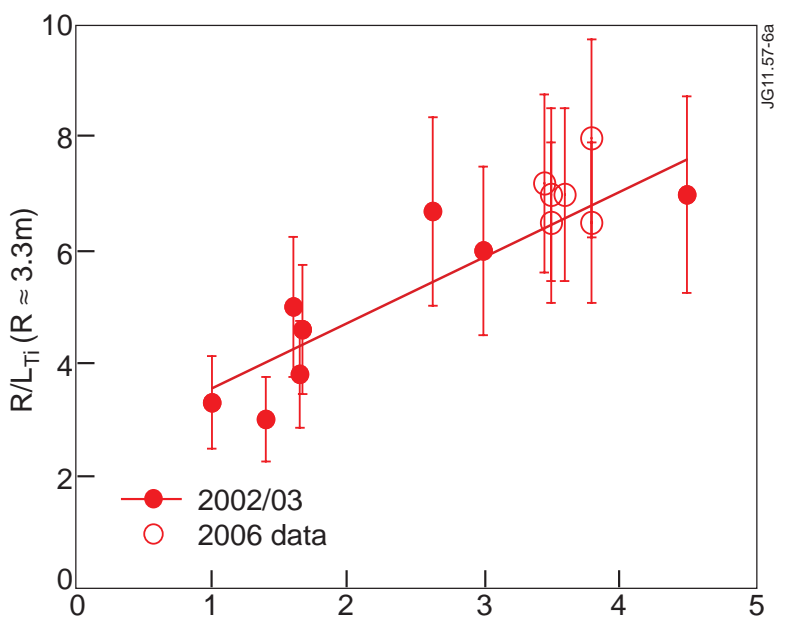

$\omega_{\text {tor }}(R \approx 3.3 \mathrm{~m})\left(10^{4} \mathrm{rad} / \mathrm{s}\right)$

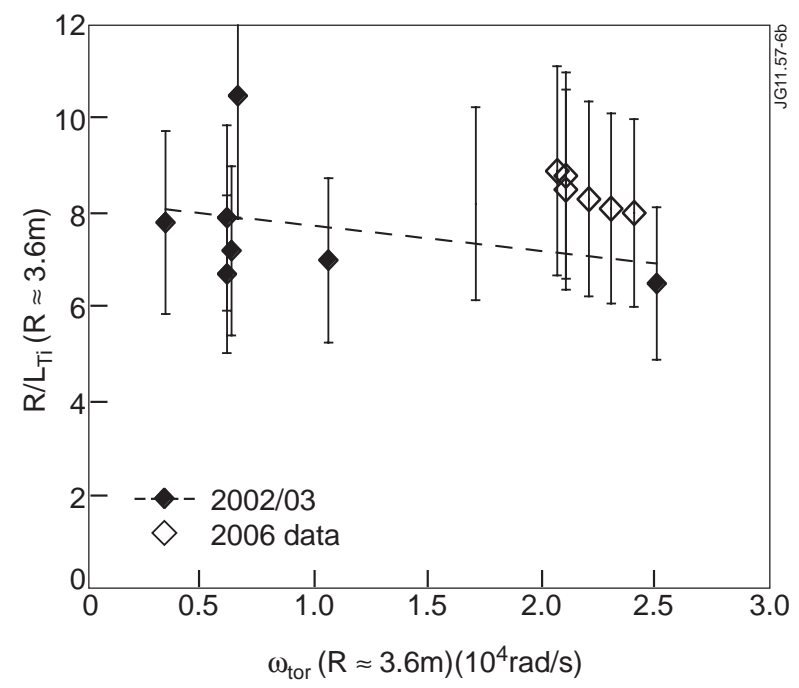

Figure 6. Experimental results from the 2002, 2003 and 2006 series: $R / L_{T_{i}}$ in the inner part and outer part of the plasma around $R \approx 3.3 \mathrm{~m}$ and $R \approx 3.6 \mathrm{~m}$ versus toroidal angular rotation at these respective positions. The lines are linear least square fits to the 2002 and 2003 data.

The heat pulse analysis for the $4 \mathrm{~Hz}$ modulation can be performed for the inner part, around $R=3.3 \mathrm{~m}$. The results as a function of $R / L_{T_{i}}$ and toroidal rotation are plotted in Fig. 7. Within the large experimental uncertainties, they suggest a trend for the normalised heat pulse propagation, $\propto \chi_{i}^{H P} T_{i}^{3 / 2}$, to decrease with increasing $R / L_{T_{i}}$ and rotation, as in [17]. The large error bars are due to the fact that only 3 or $4 T_{i}$ measurement points were available in the required radial range in the 2002 and 2003 campaigns.

These experimental data indicate that the discharges are reproducible from one campaign to the other and that the data for three experimental series are consistent. In particular, the 

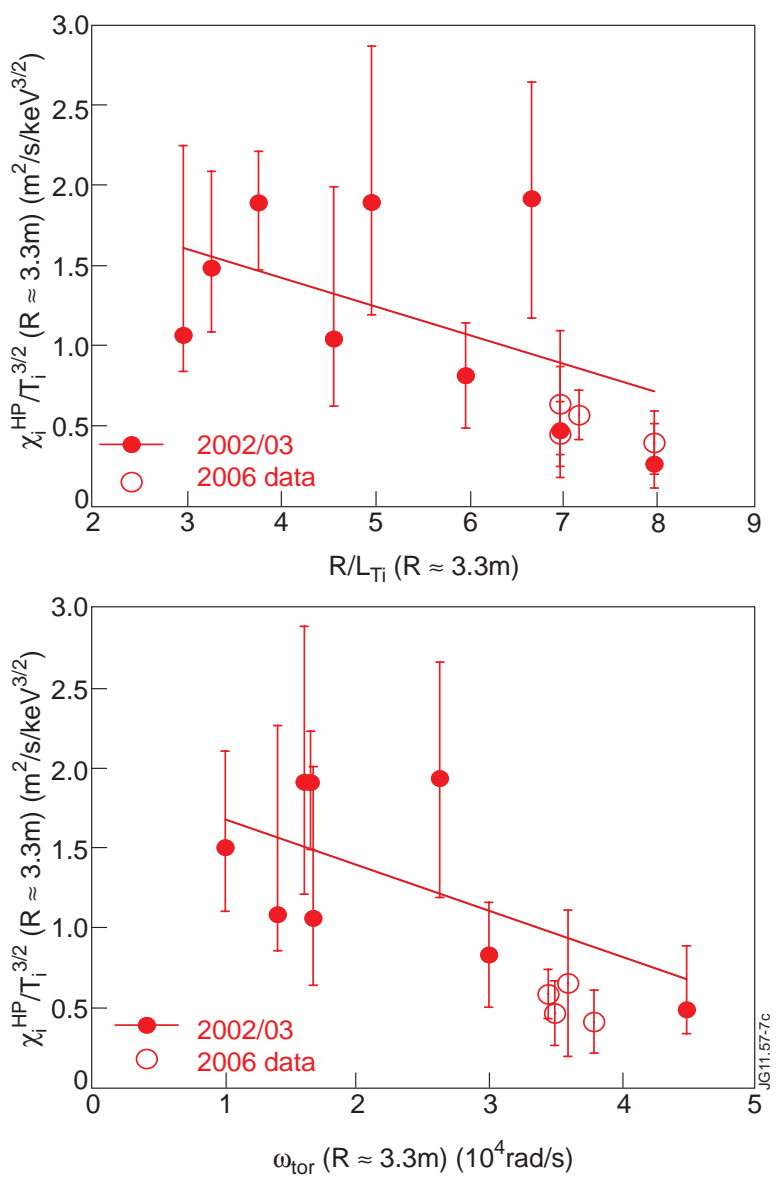

Figure 7. Experimental results from perturbative transport analysed around $R \approx 3.3$ $m$ for the 2002, 2003 and 2006 series. Normalised values of $\chi_{i}^{H P}$ versus $R / L_{T_{i}}$ and toroidal angular frequency. The lines are linear least square fits to the 2002 and 2003 data.

2006 discharges do not deviate from the 2002 and 2003 series. This allows us to focus the modelling on these discharges which offer the best $T_{i}$ measurements and the scan in modulation frequency, with the guarantee that they are representative of such experiments.

\section{Modelling approach}

In this section we describe the approach chosen to model these investigations. The electron and ion heat sources from NBI and ICRH are calculated taking into account the timedependent experimental wave form of the RF power modulation, as described below. For NBI, the heat sources are provided by the TRANSP code and its NUBEAM routine,[34]. For the ICRH, we used the PION and SELFO codes, described in the next sub-section. Heat 
transport is described by a simple model with critical gradient hypothesis combined with results from non-linear gyro-kinetic calculations carried with out with the code GYRO. We chose this approach because modelling with the GLF23 transport code [35] yielded results for which stiffness and heat transport were too high by about a factor of two. The transport simulations themselves are performed with the ASTRA transport code, [36], in which the critical gradient model is implemented.

\subsection{Modulated heat sources by ICRH}

In the ICRH ${ }^{3} \mathrm{He}$ minority scheme chosen for these experiments, the main part of the heating occurs through collisions of the RF-accelerated ${ }^{3} \mathrm{He}$ ions with the main plasma species. The faster ${ }^{3} \mathrm{He}$ ions deliver their energy to the electrons, with a long time constant, whereas the slower ones provide ion heating, with a shorter time constant. The respective power densities are $P_{e, c o l l}$ and $P_{i, \text { coll }}$, peaked around the radial position of the ICRF resonance. In addition, direct electron heating by the fast wave occurs, without any time delay, yielding a centrally peaked deposition, $P_{e, \text { direct }}$. In this work, we used the codes PION [24] and SELFO [25] to calculate the ICRH deposition and the power transfer to the plasma. The collisional energy transfer of the RF-accelerated ions is treated differently in the two codes. The PION code uses simplified Fokker-Planck calculations, whereas in SELFO the FIDO Monte-Carlo code is used, which is more accurate. The PION calculations are time-dependent, according to the experimental RF power modulation wave form. The time resolution of the calculation is $15 \mathrm{~ms}(67 \mathrm{~Hz})$, sufficiently fast compared to the maximum modulation frequency of $20 \mathrm{~Hz}$ used in this work. The SELFO calculations were not time-dependent.

The time-averaged power density profiles for a representative shot of the frequency scan are indicated in Fig. 8. In these discharges, $P_{i, \text { coll }}$ and $P_{e, \text { coll }}$ are deposited somewhat off-axis, with a maximum at $\rho_{t o r} \approx 0.2$, whereas $P_{e, \text { direct }}$ is indeed peaked on the plasma axis. The time-averaged contributions to the total ICRH heating power calculated by SELFO and PION are compared in Table 1.

The total power absorbed by the ${ }^{3} \mathrm{He}$ ions and delivered by collisional transfer to the plasma is the same for the two codes. However, the ion and electron heating fractions are different. The fraction of ion heating calculated by SELFO is somewhat lower than that yielded by PION and correspondingly that for collisional electron heating is significantly larger. This is not due to different values of ${ }^{3} \mathrm{He}$ but reflects the different treatment of 


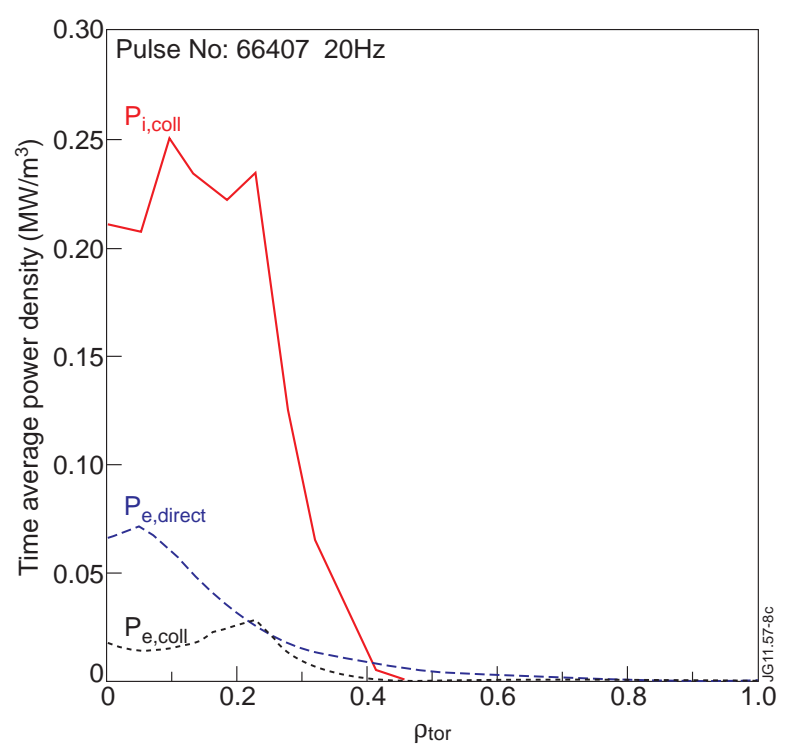

Figure 8. Results from PION for modulated ICRF. Time-averaged profiles of the different deposited power densities.

\begin{tabular}{|c|c|c|c|c|}
\hline Channel & Power $^{\text {SELFO }}[\mathrm{MW}]$ & Fraction $^{\text {SELFO }}$ & Power $^{\text {PION }}[\mathrm{MW}]$ & Fraction $^{\text {PION }}$ \\
\hline$P_{i, \text { coll }}$ & 1.32 & 0.61 & 1.64 & 0.79 \\
\hline$P_{e, \text { coll }}$ & 0.72 & 0.33 & 0.26 & 0.12 \\
\hline$P_{e, \text { direct }}$ & 0.12 & 0.06 & 0.18 & 0.09 \\
\hline
\end{tabular}

Table 1. Time averaged powers and fractions relative to the total ICRF total heating power for the different channels yielded by SELFO and PION.

collisional energy transfer of the RF-accelerated ions in the two codes, as mentioned above. We assume the values for $P_{i, c o l l}$ and $P_{e, \text { coll }}$ from SELFO to be closer to reality than those from PION and use them. The magnitude of the direct electron heating power, $P_{e, \text { direct }}$, predicted by the two codes is small compared to the other contributions, but is about a factor of 1.5 larger for PION. The TORIC calculations support the higher value yielded by PION, however, with a rather high sensitivity to the ${ }^{3} \mathrm{He}$ concentration. We will see in Sect. 6 that indeed multiplying $P_{e, \text { direct }}$ from SELFO by 1.5 yields better results for electron data.

Summarising, the two codes clearly indicate that the fraction of ion heating is indeed dominant in this ${ }^{3} \mathrm{He}$ scheme which is therefore adequate for $T_{i}$ modulation experiments. However, the difference in electron heating yielded by the two codes are significant and might influence the interpretation of electron heat transport discussed in section 6.

Due to the time constants of the collisional energy transfer from the RF-accelerated ions 


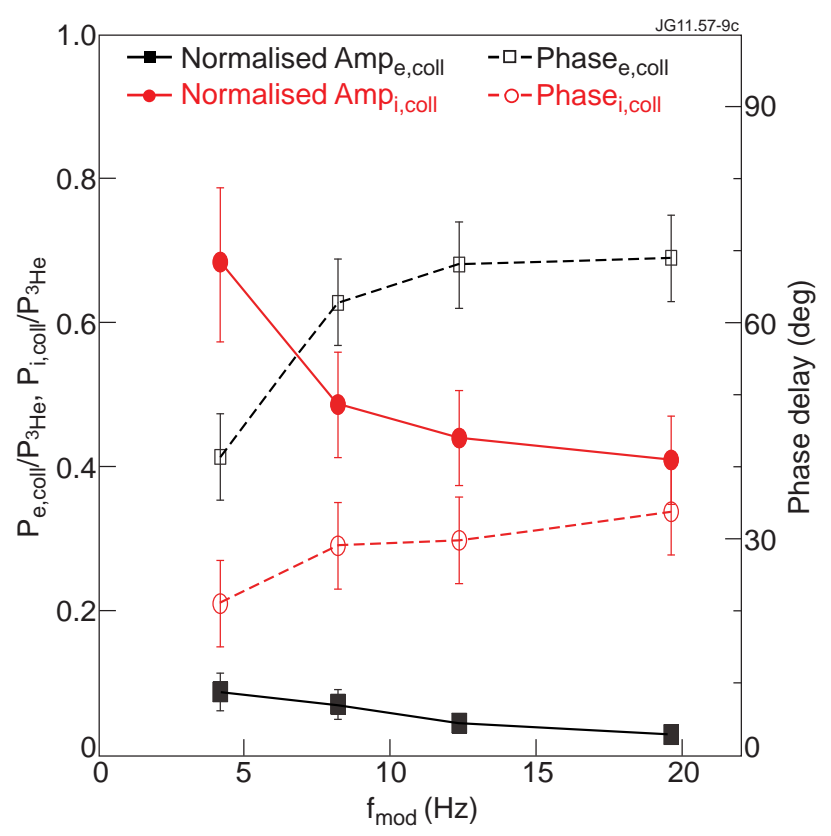

Figure 9. Results from PION for modulated ICRF. Modulation data of the volumeintegrated $P_{i, \text { coll }}$ and $P_{e, \text { coll }}$ versus $f_{\text {mod }}$ : Modulation amplitude normalised to the power absorbed by the ${ }^{3}$ He minority, and phase delay with respect to RF input power.

to the background plasma species, the modulation amplitudes of $P_{i, \text { coll }}$ and $P_{e, \text { coll }}$ decrease with increasing $f_{\text {mod }}$, whereas the phase delay with respect to the RF power increases. This is clearly illustrated by Fig. 9 where amplitude and phase delay yielded by the Fourier transform of the different heating contributions as calculated by PION are plotted versus $f_{\text {mod }}$. Note the large phase delay of $P_{e, c o l l}$, as expected from the ions with higher energies. The power transfer of $P_{e, \text { direct }}$ being instantaneous, its modulation amplitude does not depend upon $f_{\text {mod }}$ and its phase delay is always zero. These quantities are therefore not plotted in Fig. 9.

\subsection{Basic transport assumption: "critical gradient model"}

Electron and ion heat transport, induced by the TEM and ITG instabilities, increase above a threshold in normalised temperature gradient with a given stiffness. An empirical model taking these properties into account has been developed for electron heat transport and successfully tested on several devices [37, 38, 11]. This Critical Gradient Model (CGM) describes the behaviour of heat diffusivity and can be written in the same form for the electron 
and ion channels as:

$$
\chi_{j}=\chi_{j, s} q^{3 / 2} \frac{T_{j}}{e B} \frac{\rho_{j}}{R}\left[\frac{R}{L_{T_{j}}}-\frac{R}{L_{T_{j, c r i t}}}\right]^{\alpha_{j}} H\left(\frac{R}{L_{T_{j}}}-\frac{R}{L_{T_{j, c r i t}}}\right)+\chi_{j, 0}
$$

where $q$ is the safety factor, $B$ magnetic field and $H$ is the Heaviside step function which mimics the existence of the threshold. The normalised ion gyro-radius $\rho_{j}$ is $\rho_{s}=\sqrt{m_{i} T_{e}} / e B$ for the electrons and $\rho_{i}=\sqrt{m_{i} T_{i}} / e B$ for the ions. The stiffness factor is defined as $\chi_{j, s}$ and $R / L_{T_{j, c r i t}}$ is the threshold. The exponent $\alpha_{j}$, which determines the shape of the curve $\chi_{j}$ as a function of $R / L_{T_{j}}$ above the threshold, is called here "stiffness curvature". Following the results of the initial work on electron heat transport, [37], $\alpha_{j}=1$ has been used in the electron studies using the CGM, but for the ions as well, [17]. We will show below that for the ion heat transport channel $\alpha_{i} \approx 0.5$ seems to be more appropriate, in agreement with Ref. [1].

The term $\chi_{j, 0}$, which represents the transport below the threshold, is neo-classical transport for the ions. For the electrons an, arbitrary, small value is chosen. In the modelling presented below, this value is around $0.1 \mathrm{~m}^{2} / \mathrm{s}$, which is lower than $\chi_{e}$ by at least one order everywhere in the plasma, except very close to the magnetic axis where the temperature profiles drop below the threshold. In general, this term plays a weak role in transport as soon as $R / L_{T_{j}}$ is somewhat above the threshold. The gyro-Bohm factor, $T_{j} /(e B) \rho_{j} / R \propto T_{j}^{3 / 2}$, is commonly used for transport driven by micro-turbulence.

We will show in the next sub-section that electron heat transport is not only driven by the TEM but also by the ITG instability which therefore depends on $R / L_{T_{i}}$. This off-diagonal term is written above the threshold as:

$$
\chi_{i e}=\chi_{i e, s} q^{3 / 2} \frac{T_{i}}{e B} \frac{\rho_{s}}{R}\left(\frac{R}{L_{T_{i}}}-\frac{R}{L_{T_{i, c r i t}}}\right)^{\alpha_{i e}}+\chi_{i e, 0}
$$

The residual transport $\chi_{i e, 0}$ is also set to a low value of $0.1 \mathrm{~m}^{2} / \mathrm{s}$.

In contrast to electron transport, we will see below that the contribution of the TEM instability to ion heat transport can be neglected: there is no off-diagonal term for ion heat transport.

Finally, most of the previous studies using the CGM to model electron transport yielded good results assuming a linear dependence of $\chi_{e}$ versus $R / L_{T_{e}}$, i.e. $\alpha_{e}=1$. The influence of the coefficient $\alpha_{j}$ will also be discussed.

The CGM is a simple model for heat transport which is not meant to compete with elaborated physics-based models. It captures the main properties of turbulence-induced transport in the plasma core: threshold and stiffness. It can be seen as an extension of the experimental analysis, yielding quantities, such as threshold and stiffness, which can be then be compared 
to results from turbulence calculations. This is a good approach to investigate the main properties of heat transport in comparison with experimental data, in particular for modulation experiments, as demonstrated in several studies. The model runs quickly, allowing extensive scans of parameters from which the main physics information can be extracted by comparison with the experimental results.

\subsection{Non-linear gyro-kinetic calculations}

Based on the experimental data of the 2006 series, non-linear gyro-kinetic calculations have been carried out with the GYRO code, [39], adopting the local flux-tube geometry. The local description can be considered as appropriate, due to the low value of $\rho^{*}=1 / 620$ of these JET plasmas. The calculations, which include collisions but not rotation, are made for the experimental values corresponding to the situation at mid-radius. The size of the box was $81 \rho_{s}$ and $113 \rho_{s}$ in the $x$ and $y$ directions respectively, with 180 radial grid points and 32 toroidal modes up to poloidal wave number $k_{\theta} \rho_{s}=1.5$. This toroidal spectrum was found necessary for properly resolved simulations, while simulations with only 16 modes up to $k_{\theta} \rho_{s} \approx 1$ were giving significantly different results. We verified that simulations with 64 modes up to $k_{\theta} \rho_{s} \approx 2$ yield results very similar to the 32 mode cases. We also included the effect of $\left[{ }^{3} \mathrm{He}\right]$ which contributes through two effects: the dilution of the ion density and the induced change in ion density gradient, $R / L_{n_{i}}$. In the results shown below we assumed $\left[{ }^{3} \mathrm{He}\right]=15 \%$ with a flat profile which somewhat reduces stiffness and transport with respect to the case with $\left[{ }^{3} \mathrm{He}\right]=0$. We investigated, at constant temperature, the dependence of the heat fluxes $q_{e}$ and $q_{i}$ upon $R / L_{T_{i}}$ and $R / L_{T_{e}}$ around the experimental values, $R / L_{T_{i}}=5$, $R / L_{T_{e}}=8.4$ and $R / L_{n_{e}}=2$. The experimental values of $T_{e}$ and $T_{i}$ are comparable, but the profile shapes are different yielding the different gradients. The analysis of the GYRO results reveals that heat transport is dominated by the ITG instability in these plasmas, with a weak contribution from the TEM at the nominal value of $R / L_{T_{e}}$. The dependence of $q_{i}$ and $q_{e}$ on $R / L_{T_{i}}$ and $R / L_{T_{e}}$ are plotted in Fig. 10 where the points are the single GYRO results, encircled are those corresponding to the calculations made with the nominal experimental values of the normalised gradients.

The contributions of ITG and TEM driven turbulence to the heat fluxes can be roughly estimated from the dependences of the fluxes on the gradients $R / L_{T_{i}}$ and $R / L_{T_{e}}$, respectively. Panels 1 and 4 of Fig. 10 correspond to the diagonal terms, 2 and 3 to the off-diagonal 

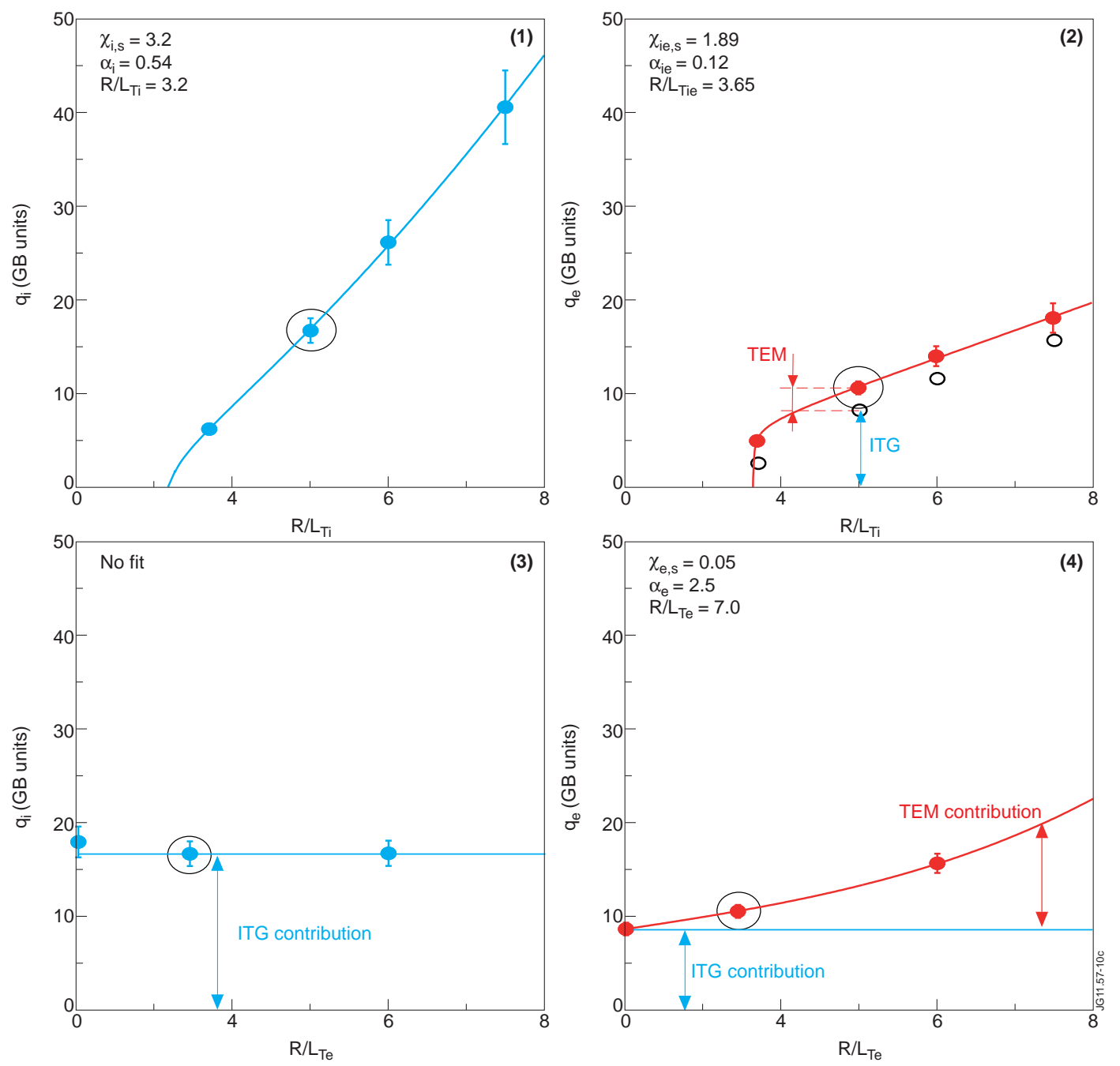

Figure 10. Results from GYRO: dependence of the heat fluxes on normalised gradients. The encircled points correspond to the nominal experimental values of the gradients. The coefficients deduced from the fits are indicated in the boxes. As indicated in the text, the off-diagonal contribution to $q_{i}$ can be negligible and no fit is done for the points of panel 3.

contributions. We assume that all four transport channels can be described by a $\chi_{j}$ function written in the form of the CGM given by Eq. 3 and 4 with respective coefficients, $\chi_{j, s}, \alpha_{j}$ and threshold which are deduced from fits to the GYRO results. The coefficients are indicated in the inserts of the respective panels. The corresponding fits for the heat fluxes, yielded by $q_{j} \propto \chi_{j} \nabla T_{j}$, are the lines in Fig. 10.

Panel 1, ion heat diffusion, shows that the dependence of $q_{i}$ on $R / L_{T_{i}}$ is close to linear, corresponding to $\alpha_{i}=0.54$ yielded by the fit of $\chi_{i}$. This dependence is in agreement with 
previous studies on ITG transport [1]. As will be discussed in the next sub-section, with $\chi_{i, s}=2.25$ the ion stiffness is rather low, which, from the point of view of our GYRO analysis, is attributed to the situation $R / L_{T_{e}}>R / L_{T_{i}}$ and, to a lesser extent, to the presence of ${ }^{3} \mathrm{He}$. Indeed, setting $R / L_{T_{e}}=R / L_{T_{i}}$ in the GYRO calculations almost doubles the stiffness value. Panel 3, which represents the contribution of the TEM instability to ion heat transport, indicates that $q_{i}$ is almost independent of $R / L_{T_{e}}$. This means that the contribution of this off-diagonal term to $q_{i}$ is negligible. We will not take this contribution into account in our modelling. Boxes 2 and 4 of Fig. 10 describe the dependences of electron heat transport. The GYRO calculations yield the total electron heat flux which consists of the sum of the contributions driven by the ITG and TEM instabilities. One can attempt to separate these two contributions by their dependences on their respective drives, $\chi_{i e}=f\left(R / L_{T_{i}}\right)$ and $\chi_{e}=f\left(R / L_{T_{e}}\right)$. The dependence of $q_{e}$ on $R / L_{T_{i}}$ exhibited in Box 2 reflects the contribution of the ITG. It is almost linear for $R / L_{T_{i}} \geq 3$.7. The clear dependence of $q_{e}$ on $R / L_{T_{e}}$ exhibited in plot 4 reflects the TEM-driven diffusion, which adds to the ITG driven flux, as sketched in this panel. The fit yields for the $\chi_{e}$ TEM contribution $\chi_{e, s}=0.05, \alpha_{e}=2.5$ and $R / L_{T_{e, c r i t}}=7$. The TEM contribution to $q_{e}$ is about $20 \%$ at the nominal experimental working point, $R / L_{T_{e}}=8.4$, as indicated in plot 4 . Therefore, the TEM contribution plays the role of a constant off-set in plot 2, if one assumes that it does not depend on $R / L_{T_{i}}$. Subtracting this off-set from the total $q_{e}$ yields the ITG contribution as schematically illustrated by the open symbols in plot 2 . The contribution of $\chi_{i e}$ to $q_{e}$ is deduced by the fit to these off-set corrected points, yielding the coefficients $\chi_{i e}, \alpha_{i e}$ and $R / L_{T_{i e, \text { crit }}}$. The results for different assumptions on the off-set, $0,20 \%$ and $40 \%$, are indicated in Table 2. The values in the first row correspond to the fit of plot 2. In addition, we perform two categories of fits, one in which the threshold value is also fitted (free $R / L_{T_{i e, c}}$ ) and one in which it is imposed at 3.2, which is the value yielded by the $q_{i}$ curve of box 1 and determined accurately. It is indeed reasonable to assume the same threshold for two transport channels driven by the ITG instability.

The values listed in Table 2 indicate that subtracting the TEM contribution from $q_{e}$ yields values of $\alpha_{i e}$ which are not very different from that of $\alpha_{i}$. In these cases with $R / L_{T_{i e, c}}=3.2$, the stiffness $\chi_{i e, s}$ for this channel is around 1.3, which is roughly half of that for the ion channel, $\chi_{i, s}=2.25$. We will see in Sect. 6 that this ratio is indeed needed to obtain good modelling results for electron heat transport. 


\begin{tabular}{|c|c|c|c|c|}
\hline TEM off-set & $\chi_{i s}$ & $\alpha_{i e}$ & free $R / L_{T_{i e, c}}$ & forced $R / L_{T_{i e, c}}$ \\
\hline 0.0 & 1.89 & 0.12 & 3.65 & - \\
\hline 0.0 & 1.58 & 0.27 & - & 3.2 \\
\hline $20 \%$ & 1.42 & 0.33 & 3.35 & - \\
\hline $20 \%$ & 1.36 & 0.35 & - & 3.2 \\
\hline $40 \%$ & 1.44 & 0.28 & 3.60 & - \\
\hline $40 \%$ & 1.14 & 0.44 & - & 3.2 \\
\hline
\end{tabular}

Table 2. Coefficients for $\chi_{i e}$ under different assumptions.

\section{Transport modelling of ion channel}

The heat transport simulations are performed with the transport code ASTRA in which the CGM is implemented for both ion and electron channels with their respective coefficients. The simulations are time-dependent using the ICRH heat sources described above. The calculated time-dependent $T_{i}$ and $T_{e}$ profiles are Fourier-analyzed, as those from experiments, and the respective amplitude and phase profiles, together with the time-averaged temperature profiles, are compared to the corresponding experimental ones to assess the quality of the modelling results. The coefficients in CGM are adjusted to yield the best agreement of the modelling results with these profiles.

In this section, we describe the modelling results for the ion temperature modulation, whereas those of the electrons are discussed in the next section. The situation for the ion channel is rather simple because there is only one source for ion heating, $P_{i, c o l l}$, and a single transport mechanism through the diffusion caused by the ITG (Fig. 10, box 1). In addition, in these discharges, the electron-ion energy exchange is weak, as can be easily checked in the transport calculations. Therefore, the ions can be modelled almost independently of the electrons, a sufficient requirement being $T_{e}$ to be within $\approx 50 \%$ of the experimental value, which also means that the ion heat wave propagation is practically not influenced by any interaction with the electrons.

For the modelling described in the following we focus on two discharges with modulation frequencies 8 and $20 \mathrm{~Hz}$. This choice is motivated by the fact that the discharges are from the same series and therefore as similar as possible, apart from the modulation frequency. As indicated above by the experimental ion data, these discharges are representative. As will 

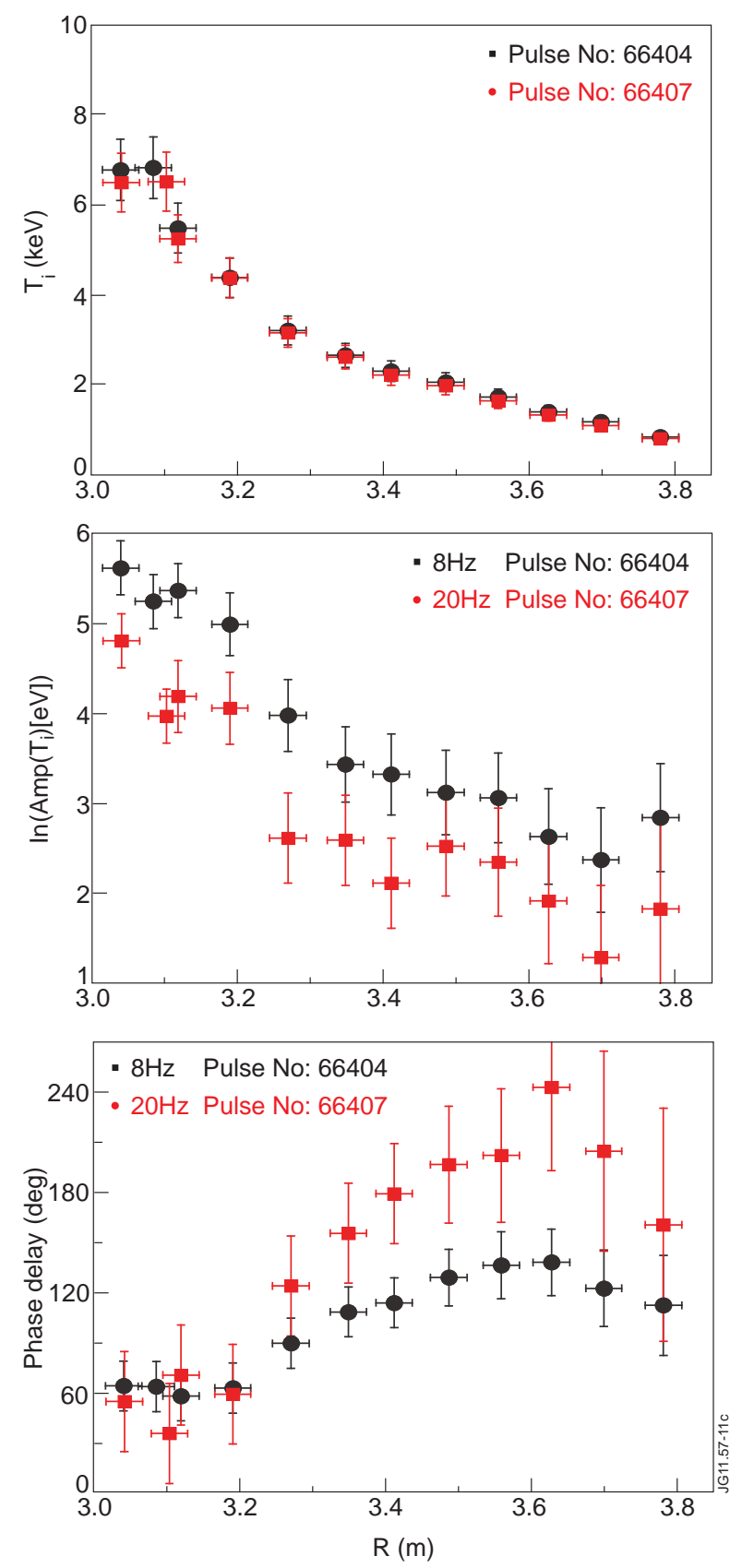

Figure 11. Profiles of experimental data of 2 similar discharges modulated at $8 \mathrm{~Hz}$ and $20 \mathrm{~Hz}$. From top to bottom: time averaged $T_{i}$ profile, natural logarithm of the Fourier amplitude, phase delay with respect to modulated ICRH power. Symbols specified in the legend.

be discussed below, the frequency of $8 \mathrm{~Hz}$ yields good conditions, sufficiently high to avoid strong influence of the edge boundary condition and low enough to provide a good signal to noise ratio $(\mathrm{S} / \mathrm{N})$. The $4 \mathrm{~Hz}$ modulation is too sensitive to the boundary condition to yield a 
reliable physics interpretation, as we indeed verified. In contrast, at $8 \mathrm{~Hz}$ its influence is low enough as shown below. The frequency of $20 \mathrm{~Hz}$, the highest we used, provides modulated data with lower $\mathrm{S} / \mathrm{N}$ than at $8 \mathrm{~Hz}$, but the quality of the data, in particular for the phase, is sufficient to deduced physics results. This is therefore an obvious choice to make optimum use of our frequency scan in the modelling study.

The relevant experimental data of these discharges are compared in Fig. 11. The timeaveraged $T_{i}$ profiles are identical. They both strongly peak in the region inside $R \approx 3.25$ $\mathrm{m}$ which is a general feature of all these discharges, as discussed later. The logarithm of the amplitude behaves as expected: it decreases about linearly from the centre to the edge and the amplitude at $20 \mathrm{~Hz}$ is lower than that at $8 \mathrm{~Hz}$. However the expected steeper slope at 20 $\mathrm{Hz}$ does not appear clearly, mainly due to the poorer $\mathrm{S} / \mathrm{N}$ linked with the lower amplitude at high frequency. In contrast, the profile of the phase delay clearly exhibits the expected steeper slope at $20 \mathrm{~Hz}$ in the region $R>3.3 \mathrm{~m}$. In contrast, the phase delay in the centre of $\approx 60^{\circ}$ is the same for both cases and this absence of frequency dependence is not in agreement with the expectation. Indeed, due to both collisional transfer of $P_{i, \text { coll }}$ (Fig. 9) and transport properties, one would expect a larger phase delay at $20 \mathrm{~Hz}$. In addition, even at $8 \mathrm{~Hz}$, this value of $60^{\circ}$ appears to be too low, as will be discussed below in comparisons between modelling results. We also note that both amplitude and phase exhibit a steep gradient in a narrow region, between the two adjacent measurement points at $R \approx 3.2 \mathrm{~m}$ and $R \approx 3.28 \mathrm{~m}$. This feature is reproducible in this series of discharges from 2006, as well as in more recent studies, [17], but not in the 2002 and 2003 data. The fact that it appears in both amplitude and phase suggests that this is not an artefact of the measurement. In addition, the $T_{i}$ profile also exhibits an increase of gradient in this region. This feature, which we call "steep gradient at $R \approx 3.25 \mathrm{~m}$ ", is discussed in detail later. We underline again here that the propagation of the temperature perturbation, reflected by $\chi_{i}^{H P}$ which depends solely on the slope of the amplitude and phase profiles at each radial position (see Sect. 2.2), is determined locally by the transport properties. Therefore, the determination of the transport properties in the confinement zone, i.e. at about mid-radius, does not depend on the modulation data in the plasma centre. This topic will be addressed in more detail later in this section.

In our modelling with CGM, the values for $\chi_{j, s}, R / L_{T_{j, c r i t}}$ and $\alpha_{j}$ yielded by GYRO serve as starting point for the coefficients used in the ASTRA simulations of the different transport channels. These coefficients are then adjusted to achieve the best match of the different 
profiles with experimental data. The agreement between experiment and theory is made by comparing these values of the different coefficients with those yielded by GYRO. It should be underlined that in adjusting the coefficients, it is essential to keep the time-averaged temperature profiles as close as possible to the experimental ones to avoid the influence of the strong gyro-Bohm dependence, $\propto q^{3 / 2} T^{3 / 2}$. Note that the transport model cannot take into account the influence of the density modulation in the very edge and the modelling results are compared to the data for $R<3.65 \mathrm{~m}$.
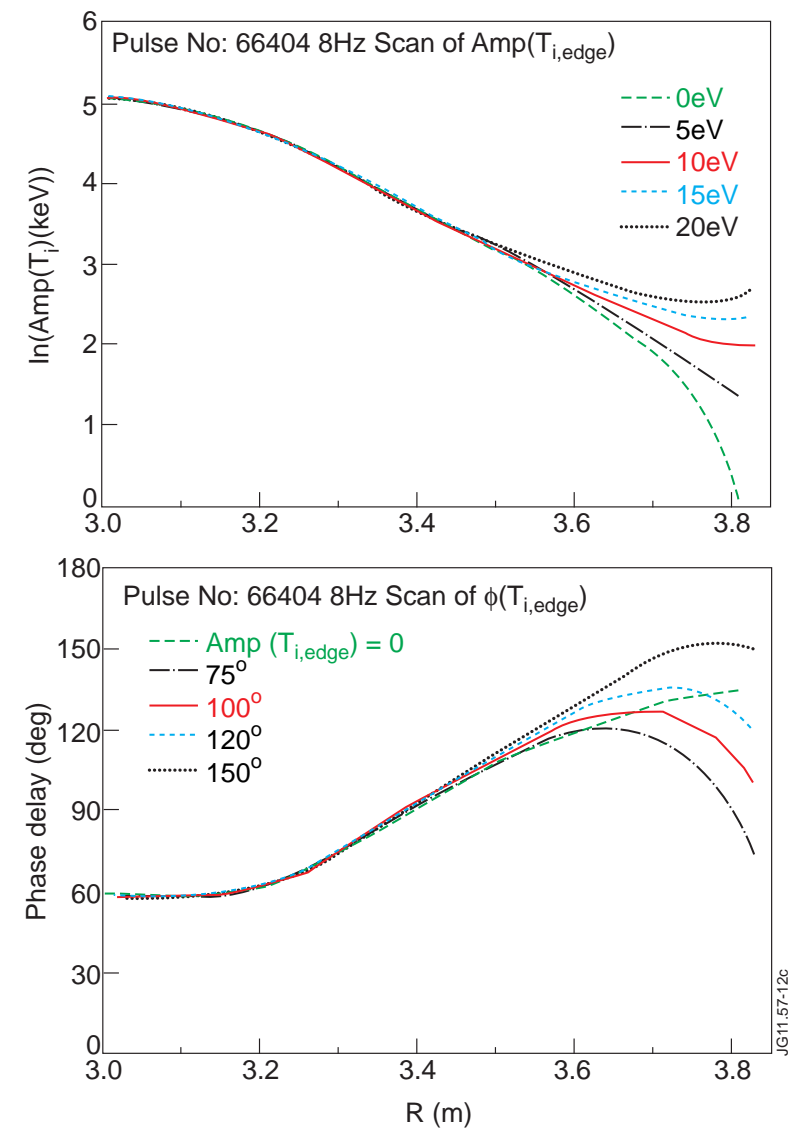

Figure 12. Effect of boundary condition on the simulated amplitude and phase of the $T_{i}$ modulation for $\chi_{i, s}=1.1$. Amplitude profile in a scan of the amplitude of $\tilde{T}_{i, B}$ for a fixed phase delay of $\tilde{T}_{i, B}$ equals to $120^{\circ}$. Profile of the phase delay in a scan of the phase delay of $\tilde{T}_{i, B}$ at a fixed amplitude of $5 \mathrm{eV}$.

However, modelling temperature modulation experiments requires an adequate timedependent boundary condition for the edge temperature. The condition used here is provided by a sine modulation of the edge temperature, $\tilde{T}_{i, B}$, with prescribed amplitude and phase delay with respect to the modulated ICRH input power. Due to the spurious effect of the edge 
density modulation, amplitude and phase cannot be modelled simultaneously in the very edge. The amplitude and phase of $\tilde{T}_{i, B}$ are adjusted to fit the data at $R \approx 3.65 \mathrm{~m}$, where the density modulation has a weak effect at $8 \mathrm{~Hz}$ and higher frequency. The effect of $\tilde{T}_{i, B}$ on the modelling results has been assessed in a scan of its amplitude and phase, shown in Fig. 12 for the $8 \mathrm{~Hz}$ case. The amplitude of $\tilde{T}_{i, B}$ affects mainly the amplitude profile of the $T_{i}$ modulation whereas its phase influences mainly the phase delay profile. Figure 12 indicates that the effect of $\tilde{T}_{i, B}$ is small for $R<3.65 \mathrm{~m}$. It should be kept in mind that the radial extent of the edge region in which the choice of the boundary condition influences the results decreases with increasing modulation frequency.

For the ion heat channel, the gyro-kinetic results presented above suggest $\chi_{i, s}=2.25$, $R / L_{T i, c r i t}=3.2$ and $\alpha_{i}=0.54$. To study the sensitivity to stiffness, we also use lower $\chi_{i, s}$ values of 1.1 and 0.6 , while higher values are not useful for the discussion, as shown below. In the following we present modelling results in which we try to improve the agreement with the experimental data making different assumption on $R / L_{T i, c r i t}$ and $\chi_{i, s}$. In particular, it turns out that assuming these two coefficients constant with radius does not reproduce properly the strong peaking of the $T_{i}$ profiles towards the center. The three following assumptions are then presented:

i) $R / L_{T i, c r i t}$ and $\chi_{i, s}$ constant over the radius,

ii) $R / L_{T i, c r i t}$ peaked towards the center,

iii) $\chi_{i, s}$ lower in the plasma center and flat $R / L_{T i, c r i t}$.

\subsection{Assuming flat $R / L_{T i, c r i t}$ and $\chi_{i, s}$}

Assuming constant coefficient over the radius and inserting them in the critical gradient model yields the results plotted in Figs. 13 and 14. For all three values of $\chi_{i, s}$, the time-averaged $T_{i}$ profile is not peaked enough and cannot reproduce the central region, $R<3.2 \mathrm{~m}$ : both $T_{i}$ and $R / L_{T_{i}}$ are too low. Further out in radius, for the case $\chi_{i, s}=0.6 T_{i}$ is clearly too steep, whereas the two cases with higher $\chi_{i, s}, 1.1$ and 2.2, are close to the experimental $T_{i}$ profile, respectively just above and just below. The amplitude profile of the modulated $T_{i}$, plotted in Fig. 14, is very roughly reproduced by all three $\chi_{i, s}$ values, within the error bars, but does not allow to determine the best case because the variations with $\chi_{i, s}$ are clearly smaller than the experimental uncertainties. In the central region, $R<3.2 \mathrm{~m}$, the amplitude from modelling seems somewhat too low. However, increasing arbitrarily the modulation amplitude of $P_{i, c o l l}$, 

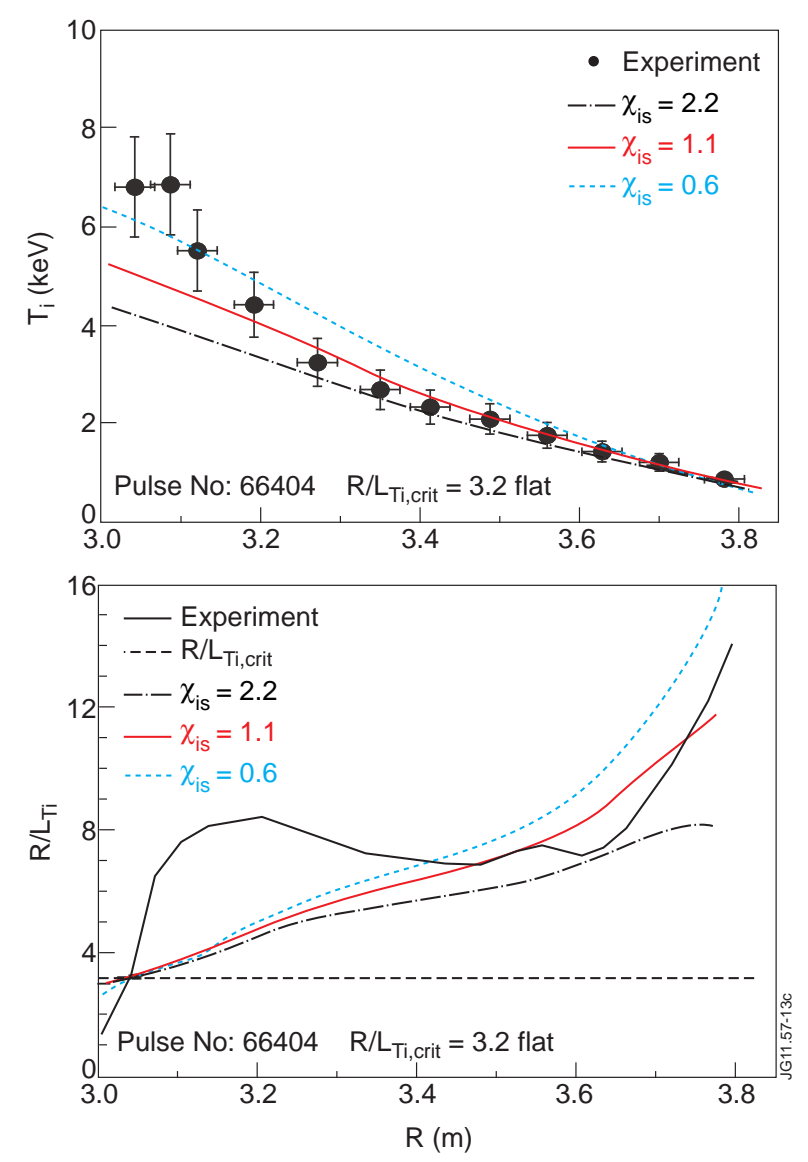

Figure 13. Time-averaged $T_{i}$ profile and normalised gradients with modelling (lines) for flat threshold $R / L_{T i, \text { crit }}=3.2$ and three $\chi_{i, s}$ values.

to match the central experimental points, using for instance another value of $\left[{ }^{3} \mathrm{He}\right]$, would vertically shift the curves upwards, leading to an overall mismatch. The higher amplitude in the central part would require lower transport in this region, as discussed below. Further out, in the region $R>3.2 \mathrm{~m}$, the modelled amplitude lies within the error bars, but with a general trend for the slope from modelling to be steeper than that of the experimental data. In addition, as our experiments were carried out at a ${ }^{3} \mathrm{He}$ concentration value close to the maximum for ion heating, an increase of the $T_{i}$ modulation in the required magnitude due to $\left[{ }^{3} \mathrm{He}\right]$ seems excluded.

In contrast, the phase delay exhibits clearly the best agreement for $\chi_{i, s}=1.1$ whereas the mismatch for 2.2 and 0.6 is consistent with that of the $T_{i}$ profile. For $\chi_{i, s}=1.1$, the match of both absolute phase value and slope is very good for $3.28<R<3.65 \mathrm{~m}$. However, as anticipated above, the phase lag in the central region, $R<3.2 \mathrm{~m}$, is higher than the experimental one by about $20^{\circ}$ and the steep gradient at $R \approx 3.25 \mathrm{~m}$ is not reproduced by 

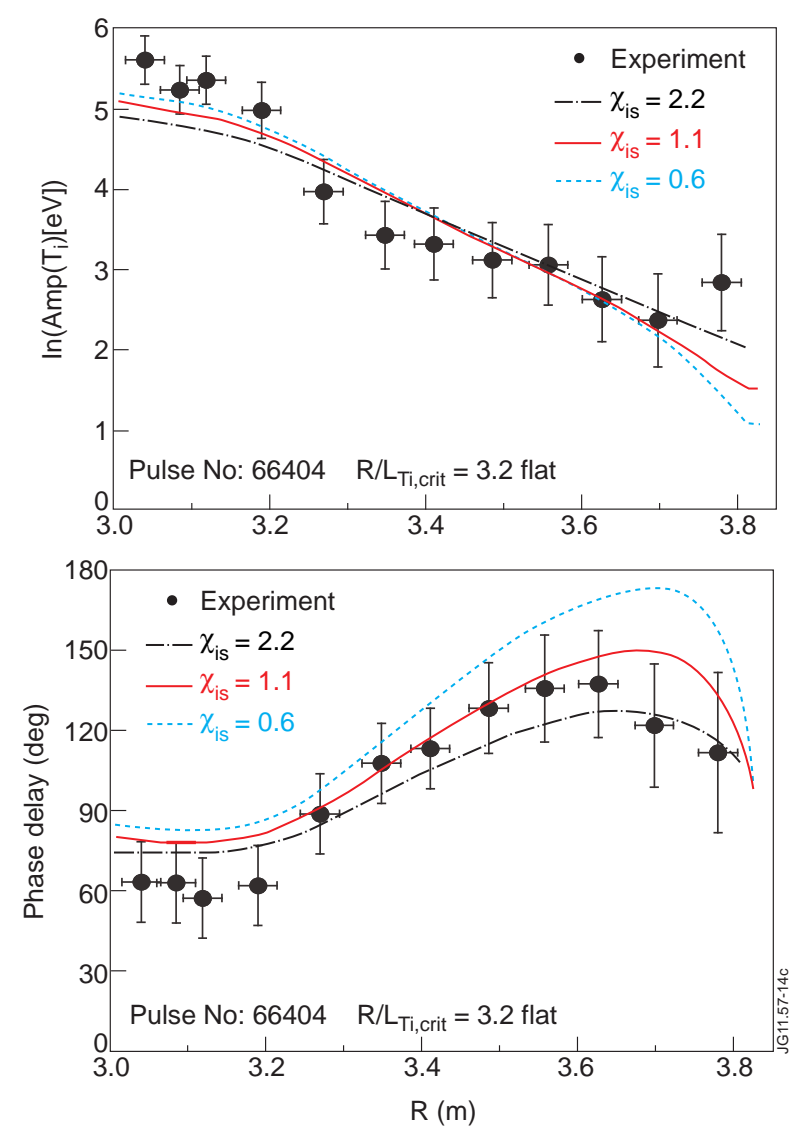

Figure 14. Modulation data for $8 \mathrm{~Hz}$, amplitude and phase delay with respect to modulated heating power, with modelling for flat threshold $R / L_{T i, \text { crit }}=3.2$ and three $\chi_{i, s}$ values.

the modelling. In the modelling, the phase delay consists of $28^{\circ}$ due to the collisional transfer (Fig. 9) and about $50^{\circ}$ caused by the plasma reaction. The latter must be at least $45^{\circ}$, [33], and the values of $50^{\circ}$ lies within the expectations. Therefore, the experimental phase delay of about $60^{\circ}$ in the centre is a rather low value considering that half of it is due to the collisional delay. Changing the central phase will be discussed at the end of this section.

In an attempt to improve the shape of the $T_{i}$ profiles and test the sensitivity of the modelling we assume a higher value for the threshold, $R / L_{T i, c r i t}=5.0$ instead of 3.2. As shown in Fig. 15, the case $\chi_{i, s}=0.6$ is clearly too high. Assuming $\chi_{i, s}=1.1$ is somewhat too high in the outer part of the profile and in rough agreement in the central part, whereas $\chi_{i, s}=2.2$ matches the experimental $T_{i}$ rather well for $R>3.2 \mathrm{~m}$. Here also, none of the three cases is able to match $T_{i}$ profile over the whole radius including its peaked central part. In comparison to Fig. 14, modulation amplitude and phase delay, shown in Fig. 15 are almost 

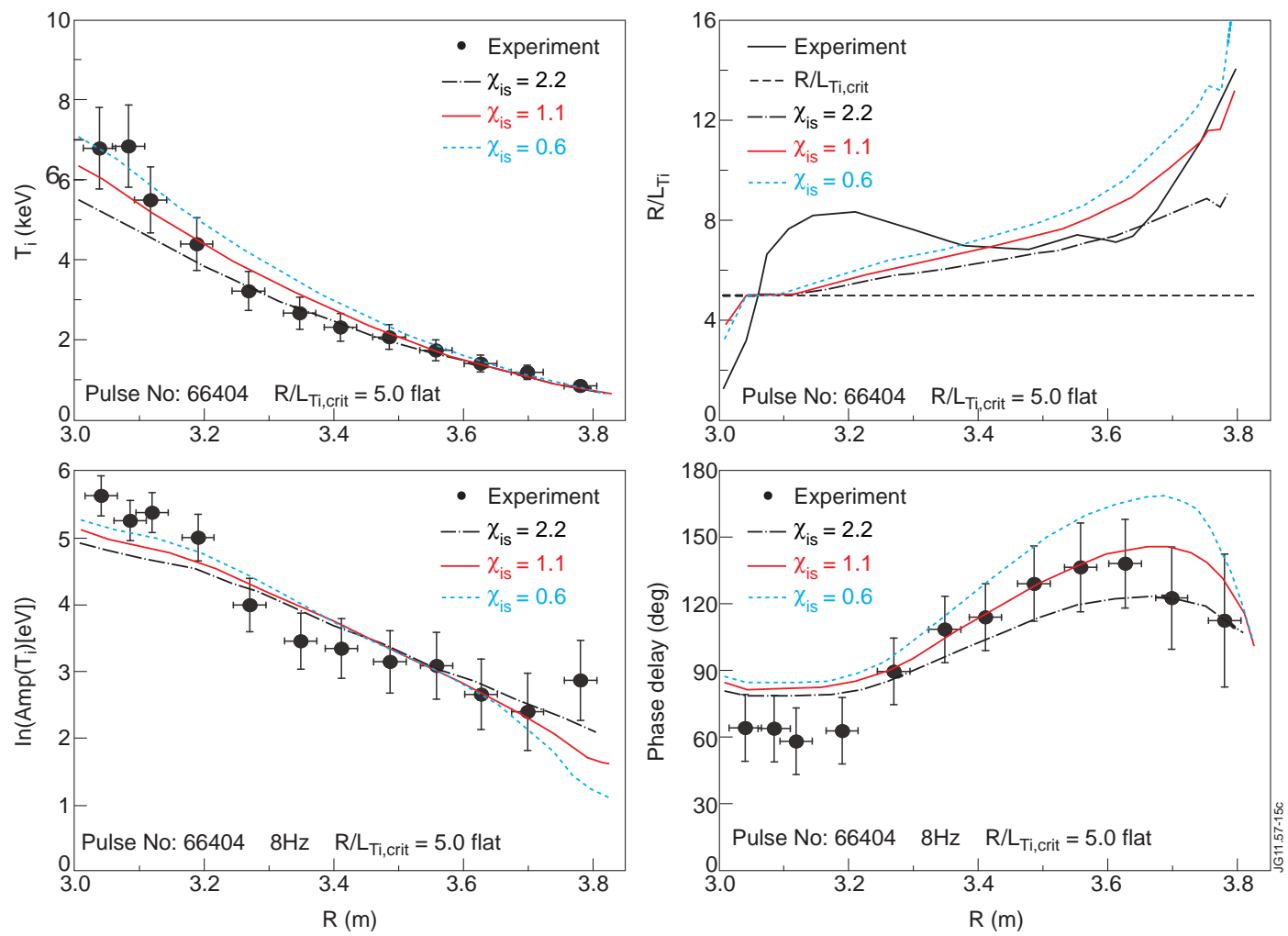

Figure 15. Time-averaged $T_{i}$ profile, as well normalised gradients, and modulation data at $8 \mathrm{~Hz}$, modelling with flat threshold $R / L_{T i, \text { crit }}=5.0$ and three $\chi_{i, s}$ values.

unchanged, yielding here also a better match for the case $\chi_{i, s}=1.1$. This indicates that the $T_{i}$ profile is sensitive to $R / L_{T i, c r i t}$ whereas the propagation of the modulated perturbation reacts weakly to changes in $R / L_{T i, \text { crit }}$.

For $20 \mathrm{~Hz}$, the same simulations have been carried out with $R / L_{T i, c r i t}=3.2$ only. For the time-averaged $T_{i}$ profile the results are very similar as at $8 \mathrm{~Hz}$, as expected for discharges with comparable controlled parameters and are not shown.

The modulation data at $20 \mathrm{~Hz}$, plotted in Fig. 16, exhibit the same features as at $8 \mathrm{~Hz}$ and lead to the same conclusions. Please note here the different scales for the phase delay at $8 \mathrm{~Hz}$ and $20 \mathrm{~Hz}$. For the amplitude at $20 \mathrm{~Hz}$, the simulations are more sensitive to the value of $\chi_{i, s}$, and the agreement improves from $\chi_{i, s}=0.6$ to $\chi_{i, s}=2.2$. As for the $8 \mathrm{~Hz}$ case, the phase delay clearly favours $\chi_{i, s}=1.1$. Here also, in the plasma centre, the phase delay from modelling is clearly larger than the experimental one.

Overall, assuming $R / L_{T i, c r i t}=3.2$, which is the value yielded by the GYRO analysis, $\chi_{i, s}=1.1$ yields a satisfactory agreement in the confinement region of the plasma, $R>3.25$ 

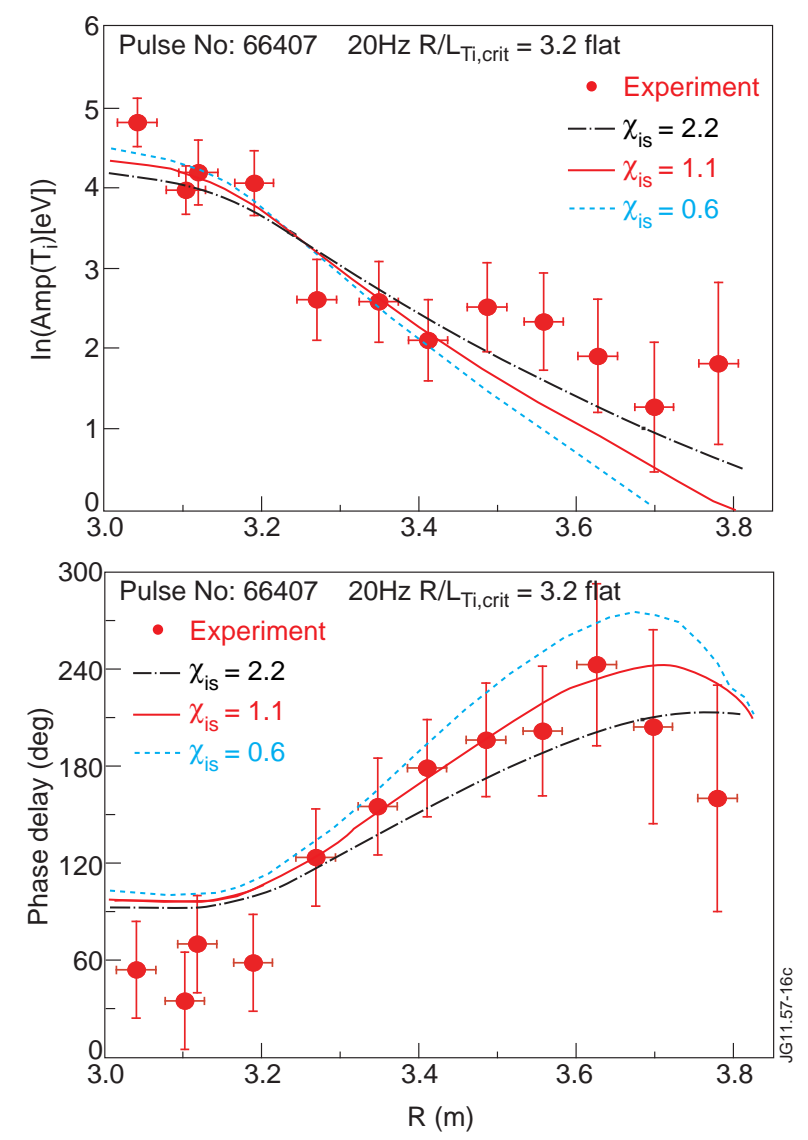

Figure 16. Amplitude and phase of $T_{i}$ modulation at $20 \mathrm{~Hz}$, modelling with flat threshold $R / L_{T i, \text { crit }}=3.2$ and three $\chi_{i, s}$ values.

$\mathrm{m}$ for both modulation frequencies. This stiffness value of 1.1 is about a factor of 2 times lower than that yielded by the GYRO calculations which were performed at $R \approx 3.4 \mathrm{~m}$. The threshold $R / L_{T i, \text { crit }}=3.2$ yielded by the GYRO calculations is adequate in the region $R>3.25$ $\mathrm{m}$, but a higher value seems to be required further inside.

\subsection{Assuming peaked threshold}

To achieve a better match in the central plasma, radially dependent profiles of threshold or stiffness factor can be investigated. The first attempt is made using a flat $\chi_{i, s}$ and a "peaked threshold", with the shape illustrated in Fig. 17, which deviates from the "nominal value of 3.2 " in the central part of the plasma.

As clearly exhibited by Fig. 18 which shows the modelling results, the central part of the $T_{i}$ profile can indeed be well matched by increasing $R / L_{T i, c r i t}$ towards the centre. This leads to higher values of both $T_{i}$ and $\nabla T_{i}$ in the central part of the plasma, such that the required 


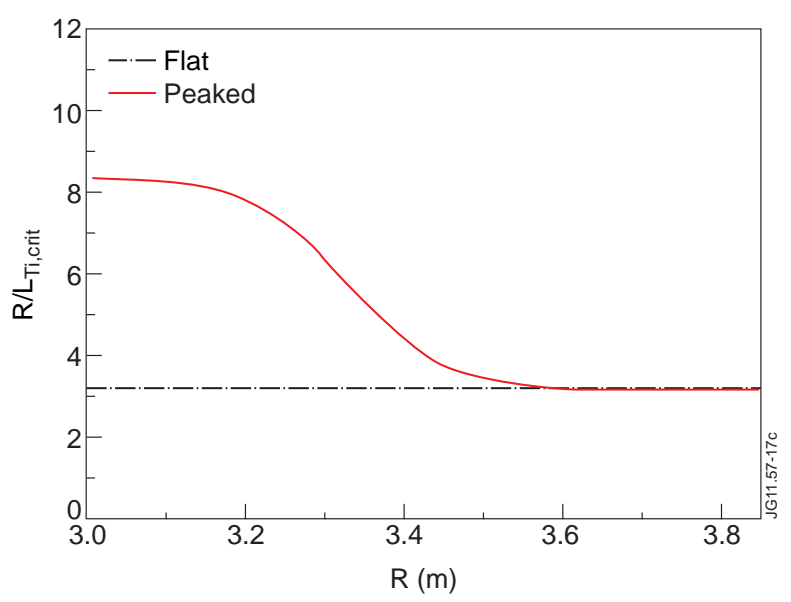

Figure 17. Flat and peaked $R / L_{T_{i, \text { crit }}}$ profiles used in the modelling described in the text.

value of the ion heat flux can be driven with a lower heat diffusivity $\chi_{i}$, as shown in Fig. 19 by the solid $\chi_{i}$ curve to be compared with the dashed line. This corresponds to a reduced value of the difference $R / L_{T_{i}}-R / L_{T_{i, c r i t}}$, as shown in Fig. 18.

In contrast, the modulation data using the peaked threshold, shown by Fig. 20 for $8 \mathrm{~Hz}$ and $20 \mathrm{~Hz}$, are weakly affected in comparison with the flat threshold case of Figs. 14 and 16. The reduction of $\chi_{i}$ in the central plasma causes only a slight increase of amplitude and phase delay in this region. The steeper gradients around $R \approx 3.25 \mathrm{~m}$ observed in the experimental amplitude and phase profiles are not reproduced. Consequently, under these conditions, the value $\chi_{i, s}=1.1$ yields here also the overall best agreement for $R>3.2 \mathrm{~m}$.

\subsection{Assuming reduced stiffness in the centre}

The second attempt to improve the agreement between modelling and experiment in the central plasma has been carried out with a $\chi_{i, s}$ profile exhibiting a lower central value, whereas the nominal value is reached for about $R>3.4 \mathrm{~m}$. We label this " $\chi_{i, s}$ hollow", for which an example for the nominal value 1.1 is shown in Fig. 21 . Note the extremely low value of $\chi_{i, s}$ in the centre required to provide good results.

The time averaged $T_{i}$ yielded by this hollow $\chi_{i, s}$ reproduces rather well the experiment as shown by Fig. 22.

Overall, the $\chi_{i, s}$ values 2.2 and 1.1 yield comparable agreement with the experiment. The profile of $R / L_{T_{i}}$ does not match the experimental profile as well as the peaked threshold in the 

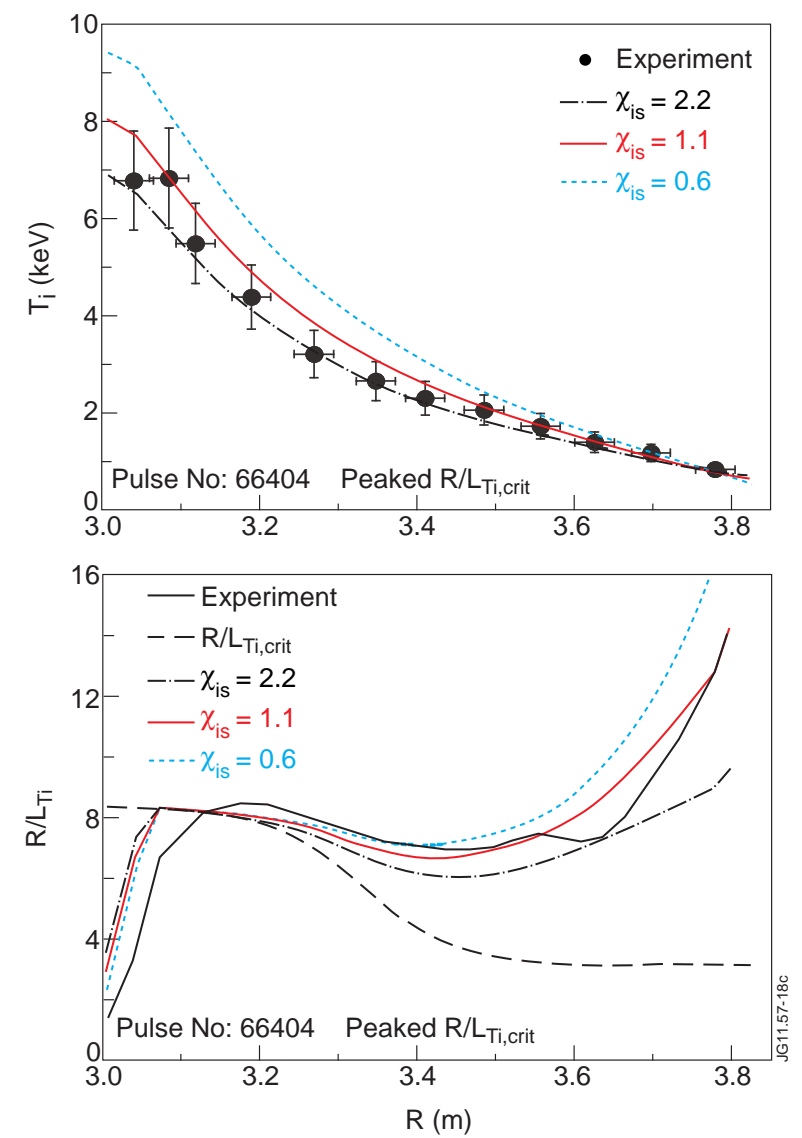

Figure 18. Time-averaged $T_{i}$ and $R / L_{T_{i}}$ profiles for a peaked $R / L_{T_{i}, \text { crit }}$ profile. Experiment and modelling using peaked $R / L_{T_{i} \text {,crit }}$ profile with nominal value 3.2 for different values of $\chi_{i, s}$, symbols and lines defined in legend.

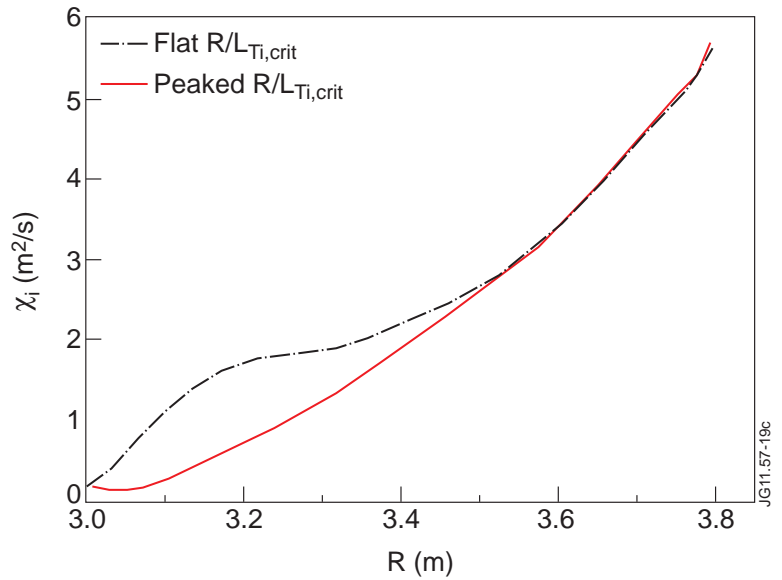

Figure 19. $\chi_{i}$ profiles corresponding to flat and peaked $R / L_{T_{i, c r i t}}$ for $\chi_{i, s}=1.1$. 

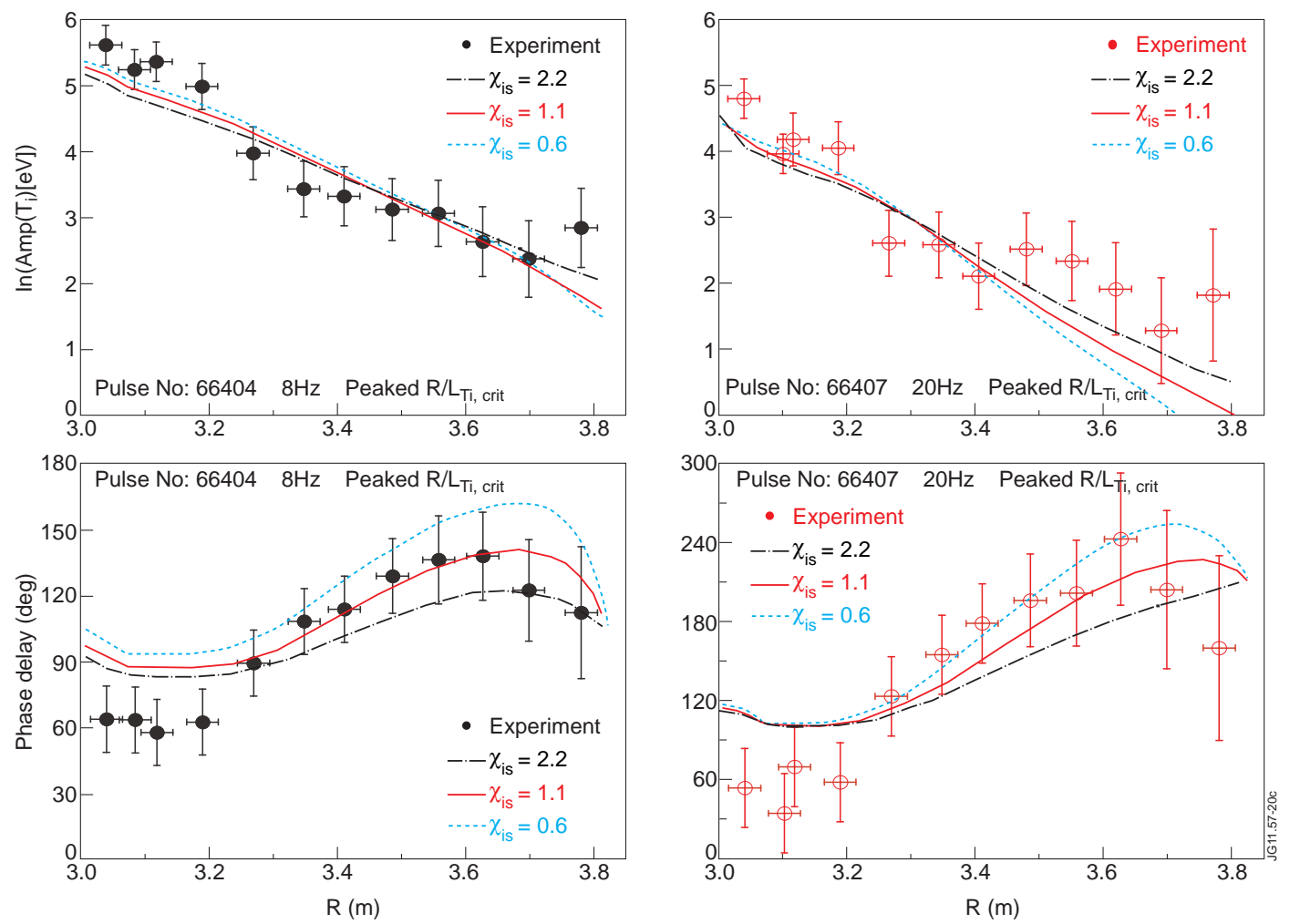

Figure 20. $T_{i}$ modulation amplitude and phase delay for $8 \mathrm{~Hz}$ in the left panels and 20 $\mathrm{Hz}$ in the right panels. Experimental data are the symbols, modelling using the peaked $R / L_{T_{i}, \text { crit }}$ profile of Fig. 18 for different values of $\chi_{i, s}$, line types defined in legend.

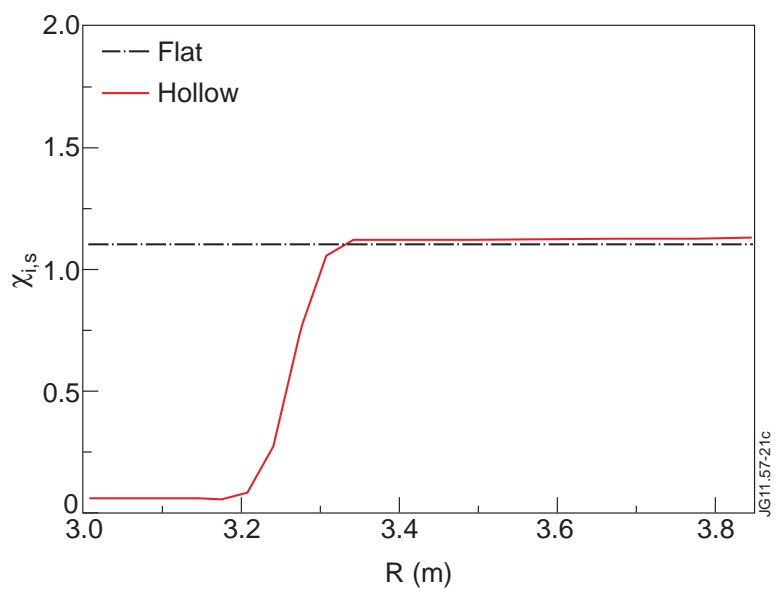

Figure 21. Flat and hollow $\chi_{i, s}$ profiles for the nominal value 1.1.

central part, the case $\chi_{i, s}=1.1$ is somewhat closer to the experiment. Here also, the higher central $T_{i}$ and $R / L_{T_{i}}$ correspond to a lower value of $\chi_{i}$, as indicated by Fig. 23

The modelling results of the modulated data for $8 \mathrm{~Hz}$ and $20 \mathrm{~Hz}$ are shown in Fig. 24. In 

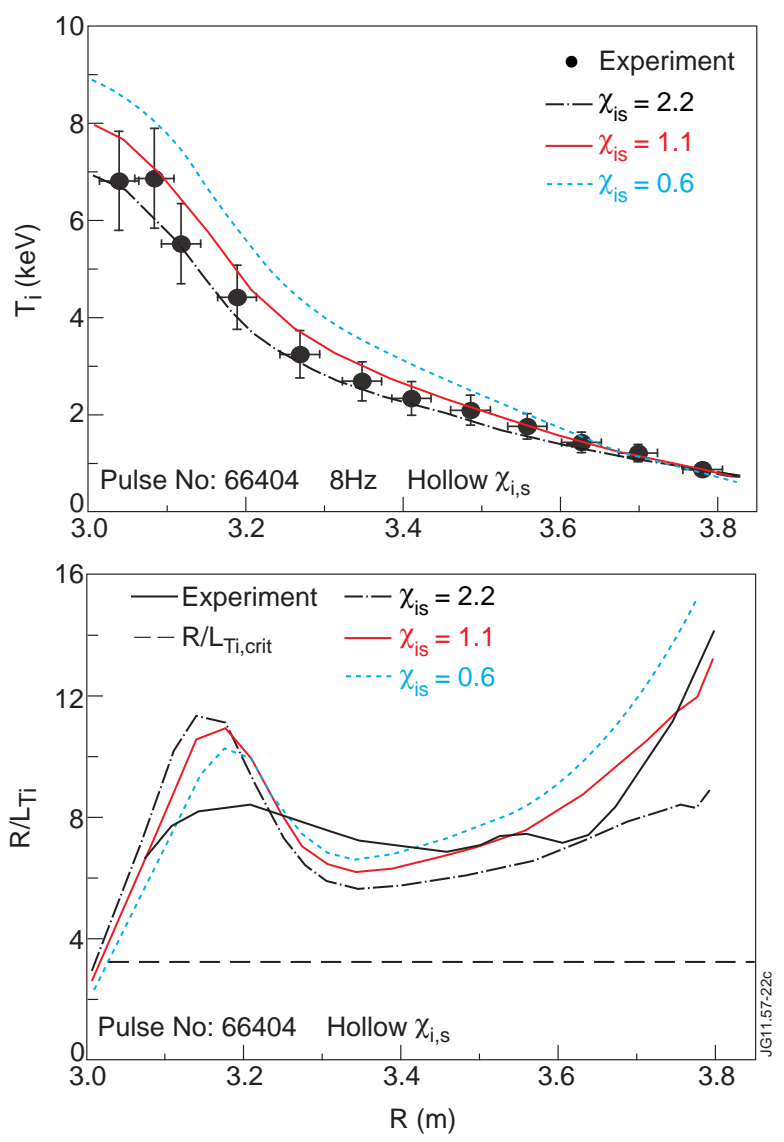

Figure 22. Time-averaged $T_{i}$ and $R / L_{T_{i}}$ profiles. Experiment and modelling using hollow profiles of $\chi_{i, s}$ similar to that of Fig. 21 with three nominal values, symbols and line types defined in legend where the indicated $\chi_{i, s}$ are the nominal values.

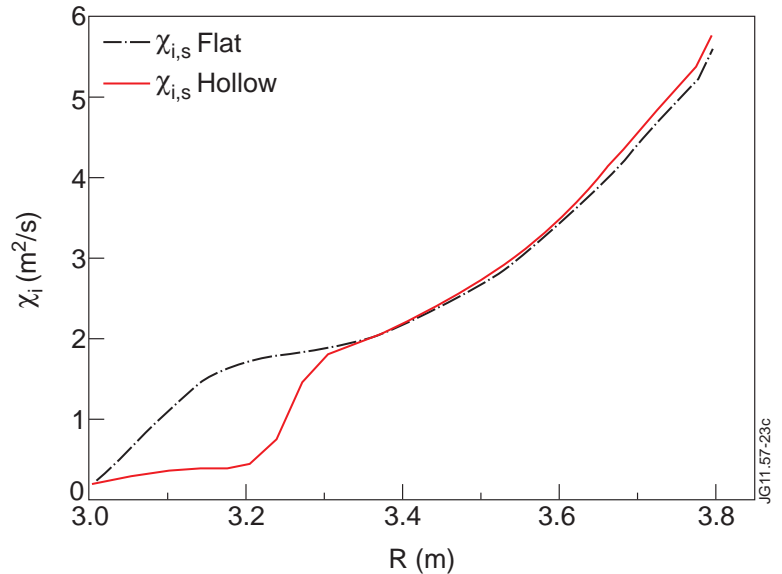

Figure 23. $\chi_{i}$ profiles corresponding to flat and hollow $\chi_{i, s}$ cases for the nominal value 1.1. 
contrast to the above results with the peaked threshold, the assumption of lower stiffness in the central plasma has a significant impact on the modulated data. This is mainly due to the the low $\chi_{i, s}$, confirming that this quantity dominates the behaviour of the heat pulse propagation.
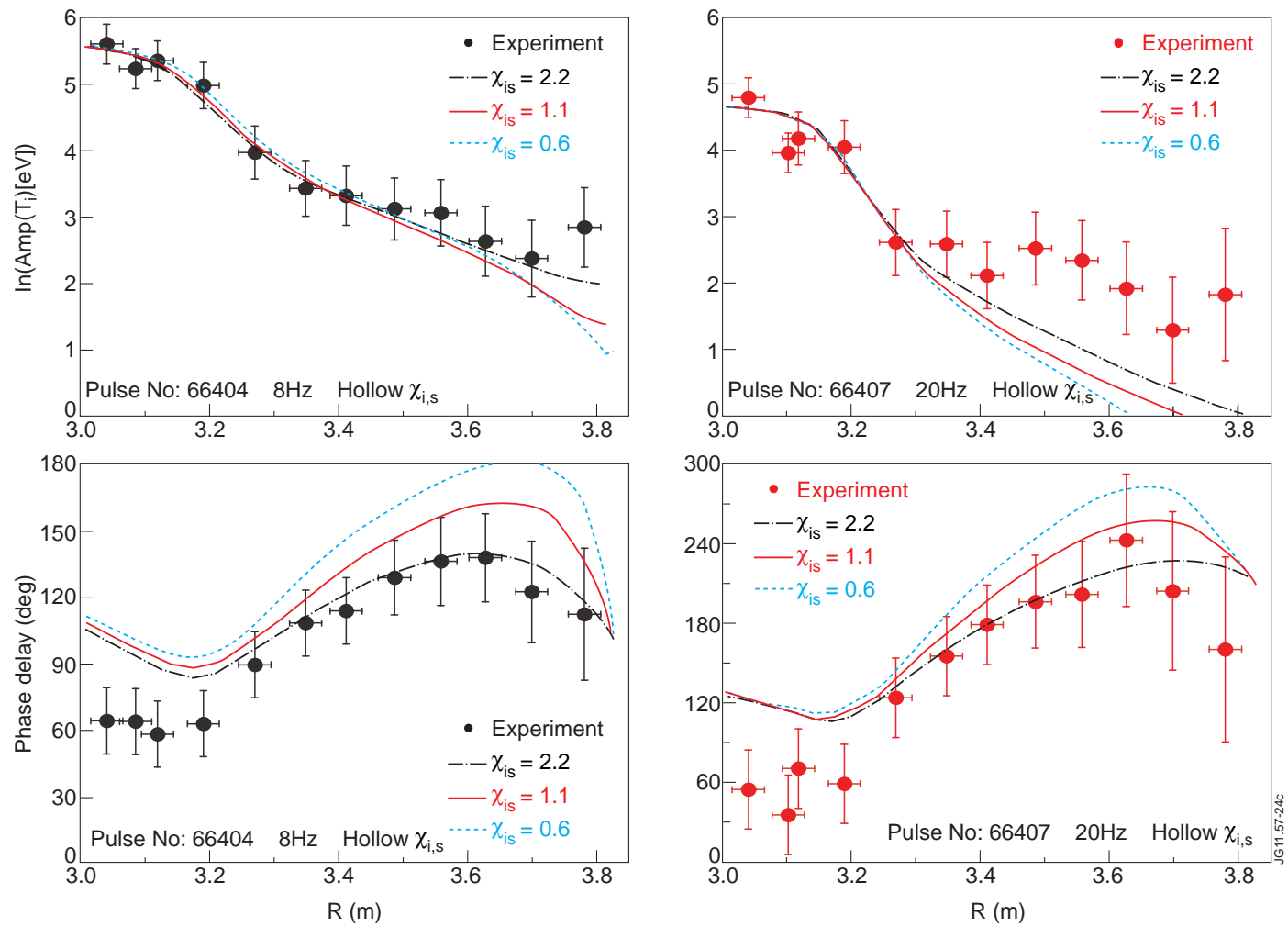

Figure 24. Profiles of $T_{i}$ modulation amplitude and phase delay at $8 \mathrm{~Hz}$ (left panels) and $20 \mathrm{~Hz}$ (right panels). Experiment data (symbols), modelling using hollow profiles of $\chi_{i, s}$ with different nominal values, lines according to legend.

The amplitude in the plasma centre is clearly larger than in the previous examples and agrees well with the experimental one. Its steeper slope around $R \approx 3.25 \mathrm{~m}$ is also rather well reproduced at both 8 and $20 \mathrm{~Hz}$. Further outside, for the $8 \mathrm{~Hz}$ case the agreement is good and the slope somewhat better reproduced using $\chi_{i, s}=2.2$. At $20 \mathrm{~Hz}$, the agreement is good from the centre up $R \approx 3.4 \mathrm{~m}$ but further outside the slope is too steep and the amplitude increasingly too low towards the edge, with a trend suggesting here also $\chi_{i, s}=2.2$ as best choice. Due to the low amplitude of the experimental $T_{i}$ modulation which is, at $20 \mathrm{~Hz}$, below $7 \mathrm{eV}$ in the region $R>3.4 \mathrm{~m}$, it is possible that our error bars are underestimated as they do not include systematic uncertainties or contribution of coherent noise. Considering now the profile of the phase delay for the two frequencies leads to the following remarks. The case $\chi_{i, s}=0.6$ clearly does not match the experimental phase delay, the slope is too steep. For 
$\chi_{i, s}=1.1$, the phase delay is too large partly due to value in the centre and due to slope which is somewhat too steep. The case $\chi_{i, s}=2.2$ yield the best agreement with the experimental data, the slope is only a bit flatter than the experimental data.

In summary, under the assumption of a hollow profile of $\chi_{i, s}$, the nominal values 1.1 and 2.2 yield the best results with a weak trend for better modelling with 2.2. In comparison with the results from the previous sub-section, a slight preference could be given to the assumption of a peaked threshold with $\chi_{i, s}=1.1$ for its more consistent agreement simultaneously with time-averaged $T_{i}$ and modulated data, at the two frequencies.

\subsection{Discussion of the amplitude and phase profiles around the ICRF power deposition}

The absence of steep gradient in the phase delay at $R \approx 3.25 \mathrm{~m}$, even assuming a hollow $\chi_{i, s}$ profile, is due to the fact that the profile of $P_{i, \text { coll }}$ extends in radius up to $R \approx 3.3 \mathrm{~m}$ where $\chi_{i, s}$ has almost reached its nominal value of 2.2. The phase delay in the region within the width of the $P_{i, \text { coll }}$ profile is dominated by the presence of the modulated power and this prevents the phase delay to exhibit the steep gradient which would otherwise reflect the low $\chi_{i, s}$ in this region. Note that assuming a hollow $\chi_{i, s}$ profile with a broader region with low value induces in the phase profile a steep gradient region which is too far out. Consequently, the steep gradient in the phase profile requires a somewhat narrower profile of $P_{i, \text { coll }}$ in addition to a low value of $\chi_{i, s}$ in this radial region.

To examine this aspect, we performed simulations with the hollow $\chi_{i, s}$ profile with nominal value 2.2 and a narrower profile of $P_{i, \text { coll }}$, plotted in the lower panels of Fig. 25 . We also attempted to achieve a better match of the phase delay in the very center and we assume a shorter time delay for $P_{i, \text { coll }}$. For this we set $\Phi\left(P_{i, \text { coll }}\right)=0^{\circ}$ for the two frequencies, instead of $\Phi\left(P_{i, \text { coll }}\right)=28^{\circ}$ at $8 \mathrm{~Hz}$ and $\Phi\left(P_{i, \text { coll }}\right)=35^{\circ}$ at $20 \mathrm{~Hz}$. This leads to the following four cases: original and narrower $P_{i, \text { coll }}$ with either original value of $\Phi\left(P_{i, \text { coll }}\right)$ or $\Phi\left(P_{i, \text { coll }}\right)=0^{\circ}$. The latter is expected to provide a better agreement with the experimental phase lag in the centre. As the total heating power is kept the same, the small change of $P_{i, \text { coll }}$ profile has almost no influence on the time-averaged $T_{i}$ profiles, which are almost unaffected and therefore not shown. The profile of the modulation amplitude, plotted in the upper row of Fig. 25, is narrower in the centre for the case " $P_{i, \text { coll }}$ narrow". It reflects directly $P_{i, \text { coll }}$ because, at such low values of $\chi_{i, s}$, the broadening of the amplitude profile by heat pulse propagation has a weak effect. Consequently, the agreement with the experimental amplitude profile is worse 

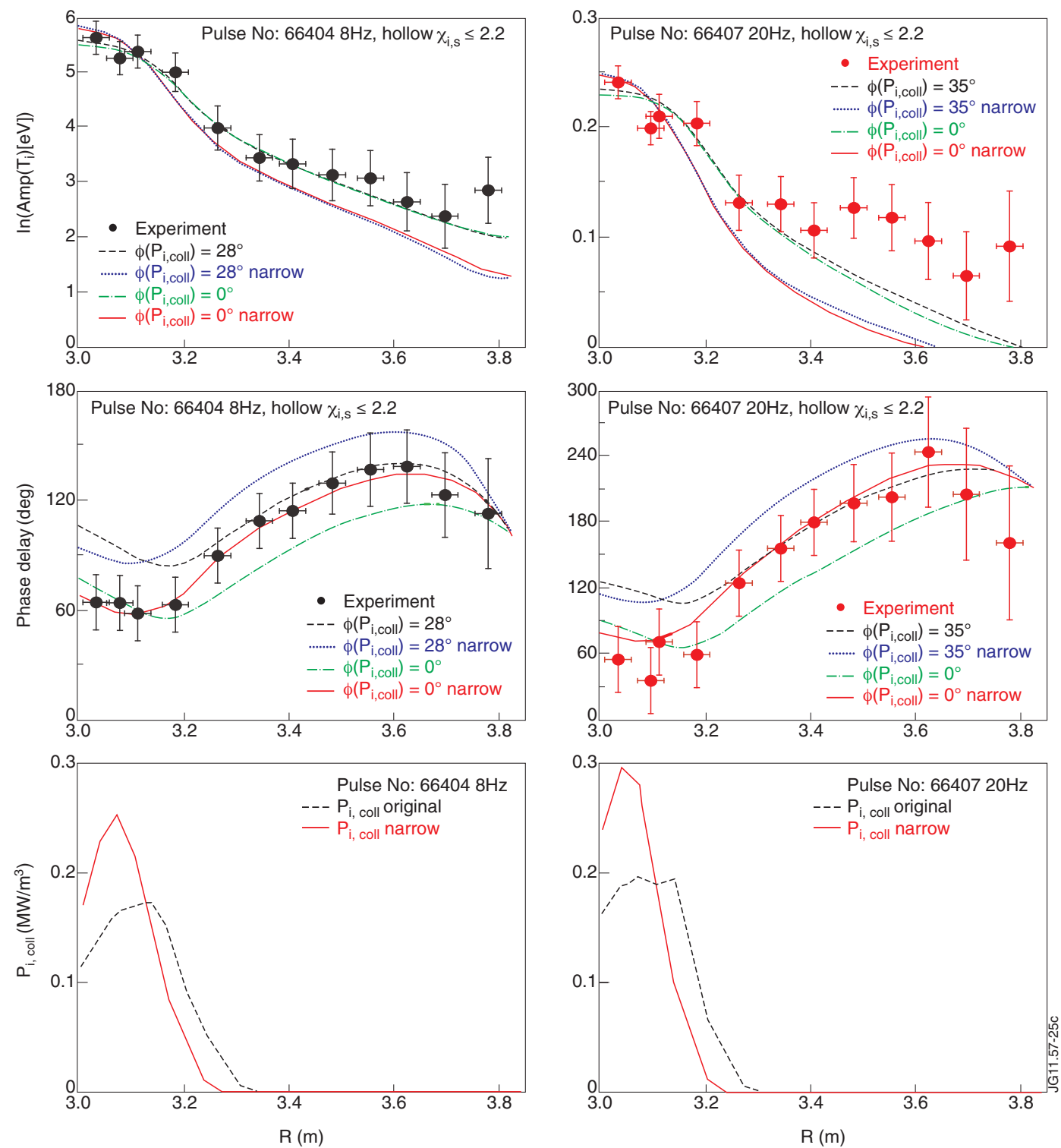

Figure 25. Amplitude and phase of $T_{i}$ modulation at $8 \mathrm{~Hz}$ and $20 \mathrm{~Hz}$. Hollow $\chi_{i, s}$ for different phase lag of modulated $P_{i, \text { coll }}$

for the narrow $P_{i, c o l l}$, with increasing discrepancy at higher $f_{\text {mod }}$. The phase delay profiles for the four cases, shown in the middle row of Fig. 25, indicate that the narrow $P_{i, \text { coll }}$ indeed allows the slope to be steeper in the region around $R=3.25 \mathrm{~m}$ and that setting $\Phi\left(P_{i, \text { coll }}\right)=0^{\circ}$ yields a good agreement in the centre for both frequencies. The combination of $\Phi\left(P_{i, \text { coll }}\right)=0$ and narrow $P_{i, \text { coll }}$ yields for the phase, at both 8 and $20 \mathrm{~Hz}$, a very good agreement with the experimental data, but the not for the amplitude. These results indicate that the best agreement with the data cannot be achieved simultaneously for both amplitude and phase with 
our assumptions, because the width of $P_{i, c o l l}$ has opposite effect on these profiles in the central part. Furthermore, although $\Phi\left(P_{i, \text { coll }}\right)$ yielded by PION certainly has some error bars, the fact that good agreement in the centre requires $\Phi\left(P_{i, c o l l}\right)=0^{\circ}$, for the two frequencies, seems well outside these uncertainties. It should be underlined that a reduction of the phase delay would require less energetic ${ }^{3} \mathrm{He}$ ions which could be provided by a significantly higher ${ }^{3} \mathrm{He}$ concentration. However, as $\left[{ }^{3} \mathrm{He}\right]$ is close to the ion heating maximum, this would strongly reduce the ion heating and cause a stronger mismatch of the modelled $T_{i}$ profile and amplitude compared to the experiment. Non-linear effects of the ICRF ion heating as a function of power in transients, might induce small collisional phase delay at low power and/or strong saturation of the heating efficiency with increasing RF power and are candidates to explain the observations. Note that such effects, which are linked with the power density, would be radius-dependent and affect the phase delay within the width of $P_{i, c o l l}$. In other words, $\Phi\left(P_{i, \text { coll }}\right)$ could be close to zero in the centre, but increase with radius up to about the value yielded by PION at the edge of the $P_{i, \text { coll }}$ profile and therefore also induce the steep slope of the phase delay around $R=3.25 \mathrm{~m}$. This would reconcile amplitude and phase behaviour with the nominal width of $P_{i, c o l l}$. Such effects depend on the ${ }^{3} \mathrm{He}$ concentration and would vary between the different discharge series. Finally, we point out that there is no reason to attribute these effects to an artefact of the measurement restricted to the central area.

In the simulations presented above, the profile of the modulated ion heating power, $P_{i, c o l l}$, has the shape illustrated by Fig. 8 for which the power is zero in the region $\rho>0.4$, i.e. $R>3.3 \mathrm{~m}$. However, even a small amount of modulated power in the outer region, $R>3.3 \mathrm{~m}$, could significantly modify the profiles of the modulated data. To investigate the sensitivity to this effect, we repeated some of the above simulations using a somewhat broader $P_{i, \text { coll }}$ profile. A 'tail' for $R>3.3 \mathrm{~m}$ has been implemented, while keeping the total heating power of $P_{i, \text { coll }}$ constant, as illustrated in Fig. 26 lower plots. In this example, the tail corresponds to about $15 \%$ of the total power. The effect on the modulation results has been investigated with the case peaked $R / L_{T_{i, c r i t}}$ and $\chi_{i, s}=1.1$ at $8 \mathrm{~Hz}$ and $20 \mathrm{~Hz}$. Figure 26 shows the resulting amplitude and phase profiles. As the total power is not changed, the amplitude is only somewhat affected in the centre but not further out. In contrast, the phase exhibits a flatter profile and the effect is stronger at $20 \mathrm{~Hz}$ than at $8 \mathrm{~Hz}$ because the modulation amplitude is smaller at high frequency and the effect of the "tail" correspondingly stronger. For the $20 \mathrm{~Hz}$ case, the phase profile for the broad $P_{i, c o l l}$ is out of the error bars and corresponds about to the case $\chi_{i, s}=2.2$ of Fig. 20 . 

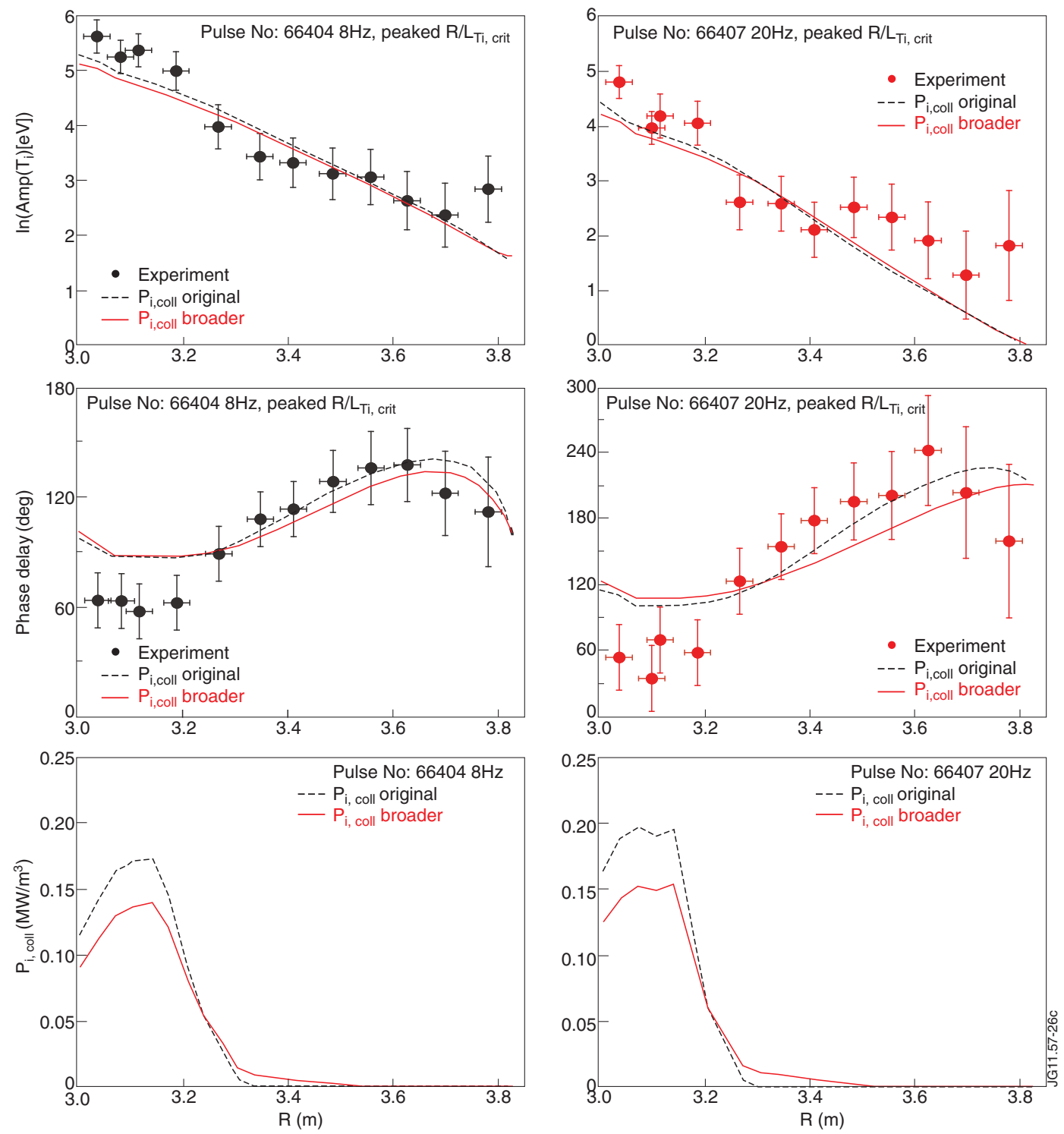

Figure 26. Amplitude and phase of $T_{i}$ modulation at $20 \mathrm{~Hz}$, for original and broader $P_{i, \text { coll }}$.

The fact that the simulations presented above, using the nominal profile of $P_{i, \text { coll }}$ and the same transport model, yield good agreement at both 8 and $20 \mathrm{~Hz}$ strongly suggests that the nominal $P_{i, c o l l}$ profiles are realistic and a significant broadening can be ruled out.

\subsection{Influence of stiffness curvature $\alpha_{i}$}

In the simulations presented so far, we used the stiffness curvature coefficient $\alpha_{i}=0.54$ as suggested by the GYRO results. However, as mentioned above, following the results of the first study with the CGM, [37], most of the studies on electron transport, as well as that on ion 
transport, [17], were carried out assuming a linear dependence $\left(\alpha_{j}=1\right)$.
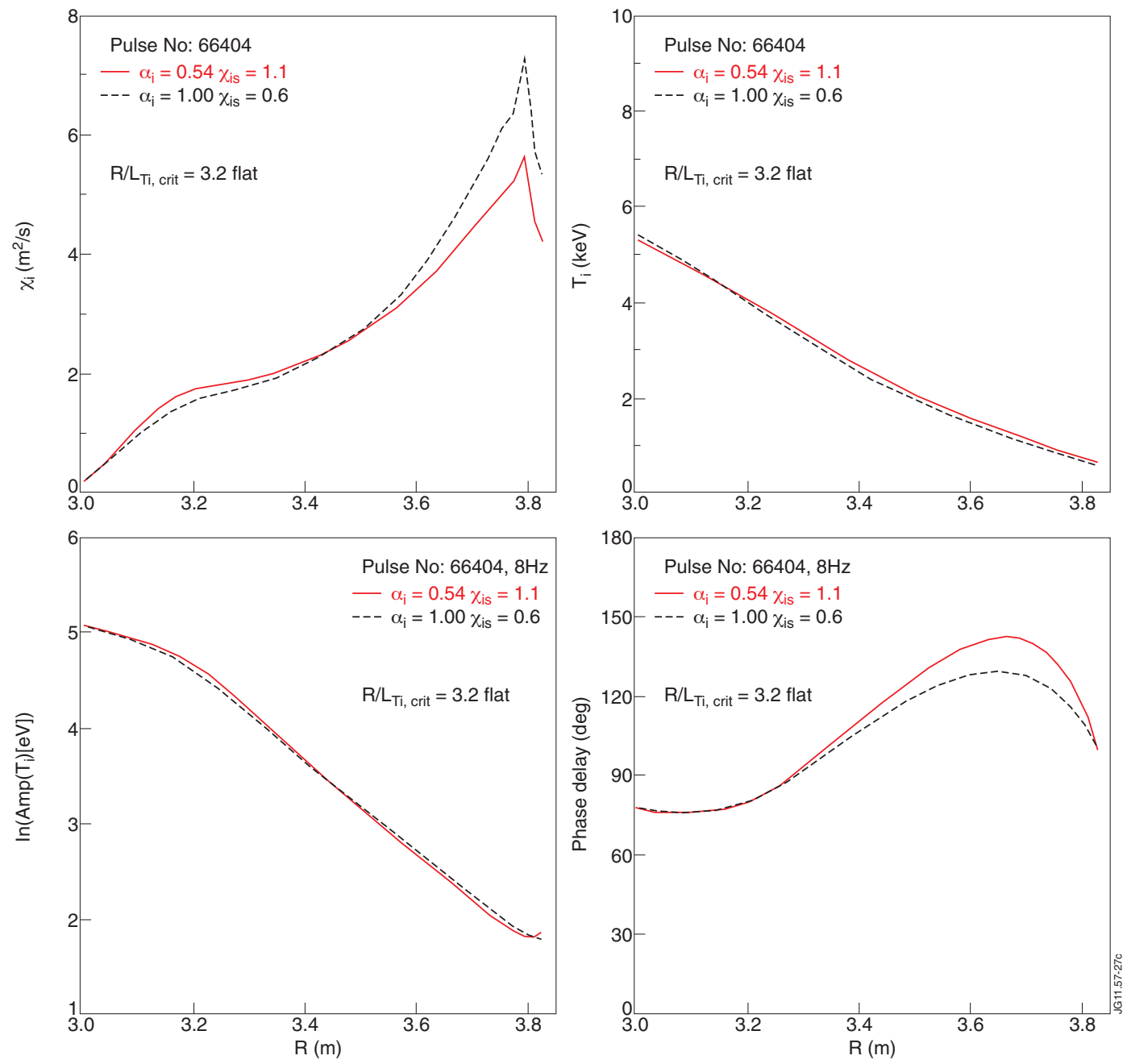

Figure 27. Simulation results with 2 values of $\alpha_{i}\left(0.54\right.$ and 1.0), in which $\chi_{i, s}$ was adjusted to yield the same values of $\chi_{i}$ at mid-radius. The $\chi_{i}$ profiles are plotted as well as the time-averaged $T_{i}$ profiles, the amplitude and phase delay of the $T_{i}$ modulation.

To compare the effect of the two assumptions for our $T_{i}$ modulation modelling, we also made simulations with $\alpha_{i}=1$ whereas the stiffness factor $\chi_{i, s}$ was adjusted such that the ion transport at about mid-radius is the same for the two assumptions. The results are shown in Fig. 27 where for $\alpha_{i}=0.54 \chi_{i, s}=1.1$ was chosen which requires $\chi_{i, s}=0.6$ for the $\alpha_{i}=1$ case. The profile of $\chi_{i}$ are somewhat different, in particular towards the edge, but the impact on $T_{i}$, amplitude and phase delay are small. This is due to the fact that, once $\chi_{i, s}$ is adjusted to provide the required transport at about mid-radius, the dependence induced by $\alpha_{i}$ has a rather weak effect in the range of $R / L_{T_{i}}$ of our discharges. This is illustrated in Fig. 28 
where the normalised $\chi_{i}$ is plotted versus $R / L_{T_{i}}$. The different dependences on $R / L_{T_{i}}$ due to $\alpha_{i}$ are clearly visible, but the induced variations in $\chi_{i}$ remain below $30 \%$, therefore within the experimental error bars in the plasma confinement zone, $R \approx 3.2-3.7 \mathrm{~m}$. The value $\chi_{i, s}=0.6$ is in agreement with those reported for NBI-heated plasmas in [17], where $\alpha_{i}=1$ has also been assumed.

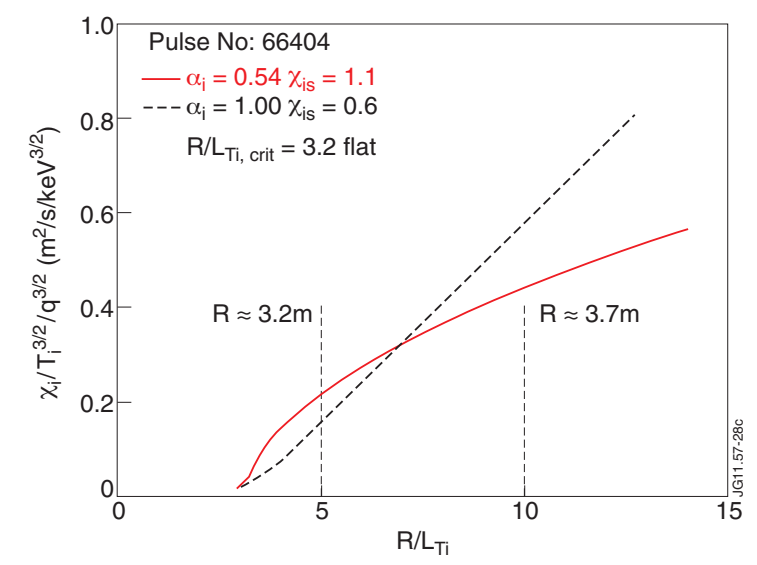

Figure 28. Simulation results with 2 values of $\alpha_{i}\left(0.54\right.$ and 1.0) and $\chi_{i, s}$ respectively 1.1 and 0.6. Diffusion coefficient $\chi_{i}$ normalised by $T^{3 / 2}$ and $q^{3 / 2}$ versus $R / L_{T_{i}}$.

\subsection{Summary of the results on ion heat transport}

Some of the modelling results presented in this section reproduce the experimental data rather well, but none of them yields an excellent agreement for $T_{i}$, amplitude and phase simultaneously. In an attempt to provide some clarity, the ability of the different assumptions to reproduce the experimental data are summarised as follows:

- flat $\chi_{i, s}$ and $R / L_{T_{i, c r i t}}$, best values $(1.1,3.2): T_{i}$ profile clearly too flat, amplitude and phase profile acceptable.

- flat $\chi_{i, s}$ and peaked $R / L_{T_{i, c r i t}}$, best values $(1.1,3.2): T_{i}$ well matched, amplitude and phase profile similar to above case.

- hollow $\chi_{i, s}$ and flat $R / L_{T_{i, c r i t}}$, best values $(2.2,3.2): T_{i}$ profile in centre not as good as with peaked $R / L_{T_{i, c r i t}}$, amplitude profile in very centre well matched but much too low at 20 $\mathrm{Hz}$ in outer region.

- Match of low phase value in the very centre and steep gradient further out requires very small collisional phase delay of ion heating, hollow $\chi_{i, s}$ and narrower power deposition 
profile. In this case, the amplitude is poorly matched.

Overall, one might favour the case flat $\chi_{i, s}$ and peaked $R / L_{T_{i, c r i t}}$, but also a combination of different hypotheses is not excluded. We will show in the next section that ion transport impacts on electron heat transport and that a general assessment of the best assumptions must take the results of both channels into account. This will be then discussed in the conclusion section.

\section{Analysis of electron heat transport}

The processes involved in the modulation of the electron temperature are more complex than those of the ion channel and the results presented here are an attempt to assess the contribution of the different effects by comparing experimental results with different assumptions for modelling. The complexity of the situation is caused by the presence of at least two heat sources and two contributions to heat transport. The modulation is, for sure, excited by the two heat sources described above, $P_{e, \text { coll }}$ and $P_{e, \text { direct }}$, which have different radial profiles and time constants, Figs. 8 and 9 respectively. We remind here that $P_{e, \text { direct }}$ has no time delay with respect to input ICRF power whereas $P_{e, \text { coll }}$ is delayed by the collisional time, which is longer than that of $P_{i, \text { coll }}$. In the ${ }^{3} \mathrm{He}$ ICRF scheme, a third electron heating source might be present in the form of off-axis mode conversion power whose magnitude and radial position depends on the ${ }^{3} \mathrm{He}$ concentration, [26]. The power is deposited further off-axis as [ $\left.{ }^{3} \mathrm{He}\right]$ is increased. On the electron heat transport side, as indicated by the gyro-kinetic calculations, both the usual diffusion, diagonal term $\chi_{e}$ driven by the TEM, and the off-diagonal term, $\chi_{i e}$ induced by the ITG, may contribute. In the following, we first describe the electron experimental data for the same two discharges for which ion transport has been presented in the previous section. We then discuss the interpretation on hand of transport simulations using the CGM under different assumptions.

\subsection{Experimental data}

Whereas the variations of the experimental time-averaged $T_{e}$, during one discharge and from discharge to discharge are small, significant changes in amplitude and phase delay of the $T_{e}$ modulation are revealed by the FFT. For our cases at $8 \mathrm{~Hz}$ and $20 \mathrm{~Hz}$, this is illustrated in Fig. 29 where the experimental data are plotted for 2 time intervals in each discharge, labelled $\Delta t 1$ 
and $\Delta t 2$. The time intervals are about 1.5 second long, $\Delta t 1$ starts about 1 second after the begin of the ICRH power modulation, where the ${ }^{3} \mathrm{He}$ concentration has reached its pre-programmed value, and $\Delta t 2$ about 3 seconds later, towards the end of the ICRF pulse.
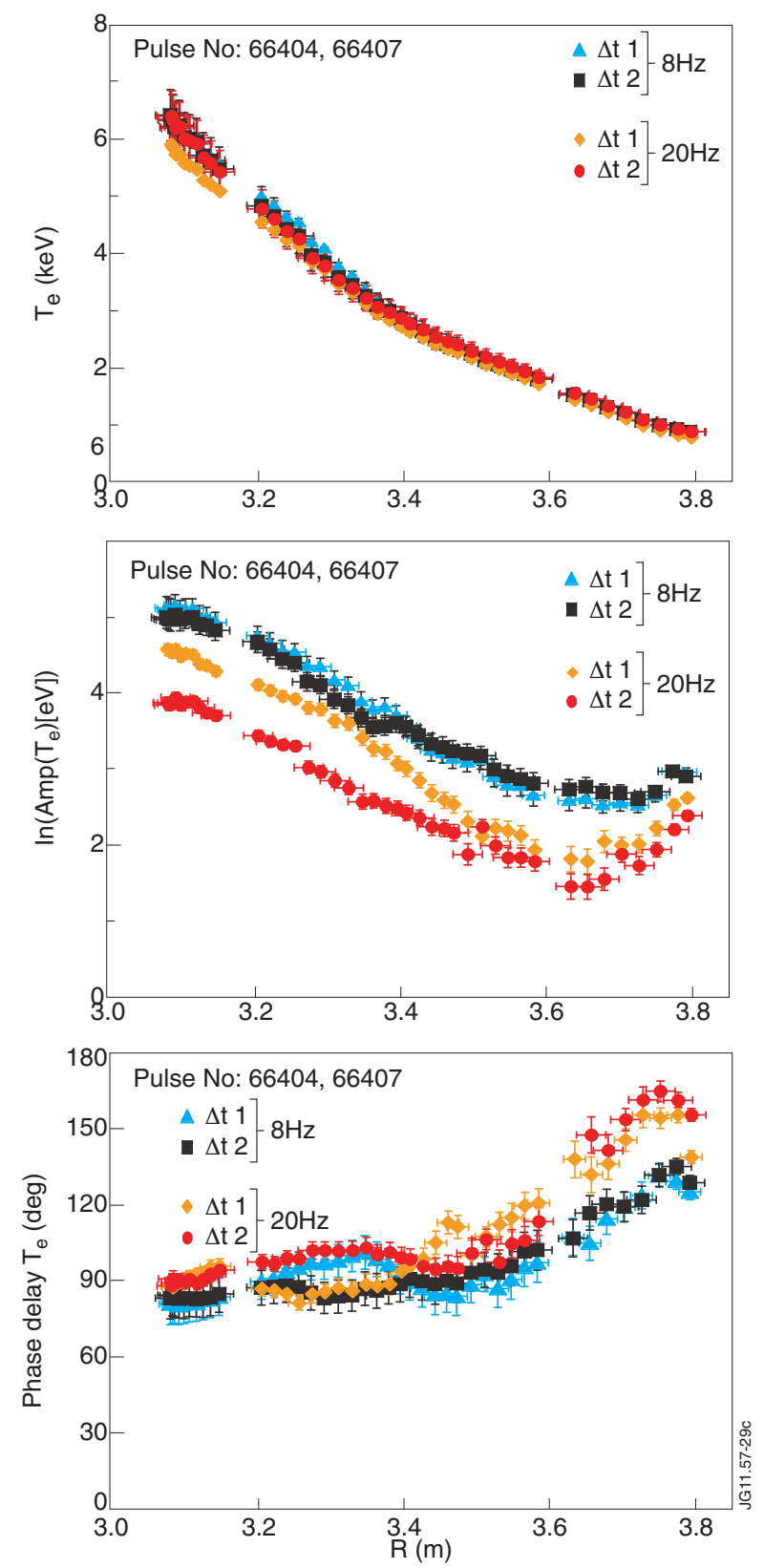

Figure 29. Experimental data for electrons for the discharges with $f_{\text {mod }} 8$ and $20 \mathrm{~Hz}$ : time-averaged $T_{e}$, amplitude and phase delay of modulated $T_{e}$. The two time intervals (see text) and frequencies are represented by the symbols defined in the legend.

As shown in Fig. 29, the time-averaged profiles of $T_{e}$ are indeed very similar for the two discharges and do not exhibit any particular feature. The modulation amplitude profiles, on 
natural logarithmic scale, exhibit the commonly observed shape, decaying roughly linearly towards the edge. The effect of the density modulation discussed in the previous sections is also visible here, on the amplitude profile which is non-monotonic and increases for $R>3.7$ $\mathrm{m}$, accompanied by a decrease of the phase delay. The amplitude is lower at $20 \mathrm{~Hz}$ than at 8 $\mathrm{Hz}$, as expected, but the slope is not clearly steeper at higher frequency. At $8 \mathrm{~Hz}$, the amplitude profile almost does not depend on the choice of the time interval, whereas, at $20 \mathrm{~Hz}$, it is quite different for the two time intervals. Finally, the profiles of the phase delay are all very flat in the region $R<3.55 \mathrm{~m}$. In addition, they exhibit a very unusual non-monotonic shape with a minimum is at $R \approx 3.45 \mathrm{~m}$, or further inside $(R \approx 3.3 \mathrm{~m}$ ), depending on the time interval. Note that the radial position of the phase minimum is not directly linked to the fact that the corresponding time interval is chosen earlier or later in the discharge: data for $\Delta t 1$ at $8 \mathrm{~Hz}$ and $\Delta t 2$ at $20 \mathrm{~Hz}$ exhibit the same shape with the clear off-axis minimum at $R \approx 3.45 \mathrm{~m}$, whereas the other 2 intervals also exhibit similar profiles with the weaker minimum at $R \approx 3.3 \mathrm{~m}$. These differences are not due to the experimental uncertainties in the FFT analysis. One may conjecture that they are caused by changes of the heating properties linked with variations of the local ${ }^{3} \mathrm{He}$ concentration, but this cannot be confirmed experimentally. The changes in the time-averaged $T_{e}$ being weak, the corresponding variations in heating power must be small, as also supported by the modelling results which will be presented below. In the outer part of the plasma, $R>3.55 \mathrm{~m}$, the phase delay increases clearly and the slope at $20 \mathrm{~Hz}$ is steeper than at $8 \mathrm{~Hz}$, as expected from heat pulse propagation basic properties.

It must be underlined that the non-monotonic, or very flat, behaviour of the phase delay in the core can only be explained by the existence of an off-axis source-like effect at about the position of the minimum, with smaller phase delay than the value of the phase minimum itself at this position. There are at least two possibilities to introduce a source-like term: the existence of an actual third modulated electron heat source, for instance through mode conversion, or the effect of the off-diagonal ITG term $\chi_{i e}$. We discuss these two options in the following subsection in simulations with the critical gradient model.

These observations are valid for the electron data of the three experimental series. The $T_{e}$ profiles vary little, whereas the modulation data are more sensitive to changes which are probably linked to the electron heating power deposition, as discussed later in this section. 


\subsection{Modelling with purely diffusive electron heat transport}

As presented in the previous section, the ion transport channel, driven by the ITG, is described with the diffusion only, $\chi_{i}$, there is no off-diagonal term. For the electron heat transport, the GYRO calculations indicate that both diffusion $\chi_{e}$, driven by the TEM, and the off-diagonal term $\chi_{i e}$, driven by the ITG, should be considered. As introduced above, these transport coefficients are written in the form of Eq. 3 for electron $(j=e)$ for $\chi_{e}$ whereas $\chi_{i e}$ is given by Eq. 4.

Investigations of electron heat transport have been carried out in JET previously by means of $T_{e}$ modulation provided by ${ }^{3} \mathrm{He}$ ICRF mode conversion, [40]. The interpretation with the CGM yielded a rather high stiffness, $\chi_{e, s} \approx 1$ for $\alpha_{e}=1$, in the presence of ion heating provided by NBI. For comparison with these results, we carry out the modelling of the present discharges under two different assumptions: firstly assuming pure diffusion in the electron heat channel, as done in [40], secondly including both diffusion and off-diagonal term.

In this sub-section, we assume that electron heat transport is completely driven by the diffusion coefficient $\chi_{e}$, as for JET simulations with the CGM in the previous studies, [40]. We anticipate that the non-monotonic shape of the phase can only be reproduced if one assumes that modulated electron heating power is deposited at about mid-radius with a low phase delay. This might be qualitatively attributed to mode conversion (MC), as revealed by our TORIC calculations. This is a direct electron heating and the phase delay of this deposited power is then zero. The simulations have been carried out according to the following assumptions:

- the value of $P_{e, c o l l}^{S E L F O}$ is correct and kept fixed for all simulations;

- the value of $P_{e, d i r e c t}^{S E L F O}$ is correct or multiplied by 1.5 , the two options are compared;

- there is the possibility for off-axis electron heating power due to mode conversion, denoted $P_{M C}$. Its value is adjusted to match the flat or non-monotonic character of the experimental phase delay.

This leads to four cases to be modelled, $P_{e, d i r e c t}^{S E L F O}$ or $1.5 \times P_{e, d i r e c t}^{S E L F O}$, with or without $P_{M C}$. We also check the influence of choosing a high and a low value for electron stiffness: $\chi_{e, s}=1$ and $\chi_{e, s}=0.1$ respectively. For each stiffness value, the threshold value is adjusted to obtained a good match of the time-averaged $T_{e}$. This yields the two couples $\left(\chi_{e, s}=1, R / L_{T_{e, c r i t}}=6.4\right)$ and $\left(\chi_{e, s}=0.1, R / L_{T_{e, c r i t}}=4.8\right)$. The values of $\chi_{e, s}$ and $R / L_{T_{e, \text { crit }}}$ are constant over the radius. Finally these simulations have been performed for $f_{\text {mod }}=8 \mathrm{~Hz}$ and $f_{\text {mod }}=20 \mathrm{~Hz}$. It should 

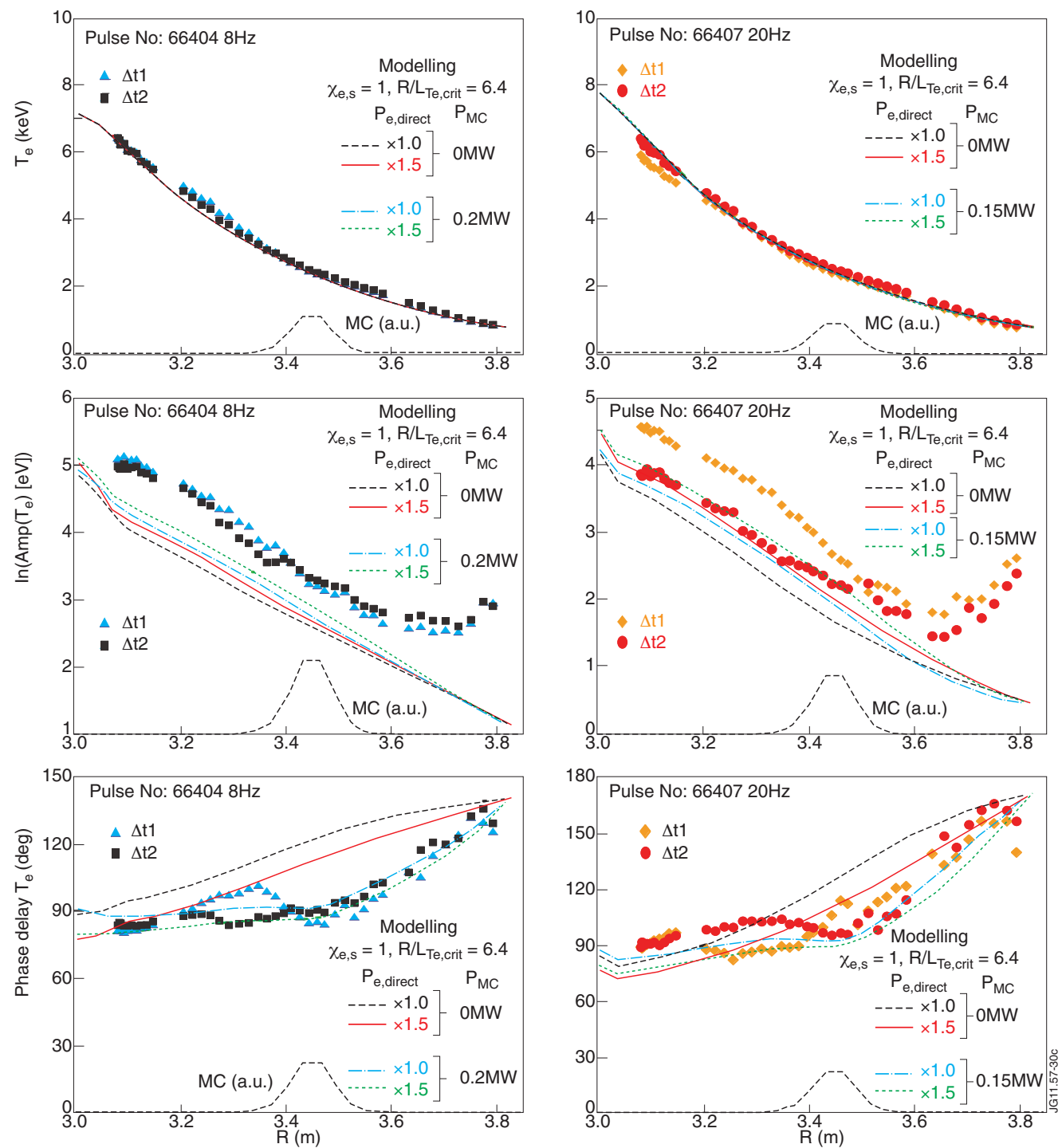

Figure 30. Time-averaged $T_{e}$, amplitude and phase of the $T_{e}$ modulation at $8 \mathrm{~Hz}$ (left panels) and $20 \mathrm{~Hz}$ (right panels). Symbols are experimental data with same coding as in Fig. 29. Modelling using pure diffusion with $\chi_{e}$ only, high stiffness cases $\chi_{e, s}=1.0$ and different assumptions on $P_{e, \text { direct }}$ and off-axis $P_{M C}$ : line types defined in legend.

be underlined that the modelling of the ion channel has almost no influence on the electron modelling in this case.

The results for $\left(\chi_{e, s}=1, R / L_{T_{e, c r i t}}=6.4\right)$ at the two frequencies are shown in Fig. 30. The $T_{e}$ profiles of the two discharges are perfectly matched for the four assumptions. In contrast to the ion case, a flat threshold $R / L_{T_{e, c r i t}}$ yields good agreement. The match depends neither on 
the variation of $P_{e, \text { direct }}$ multiplied by 1.5 , nor on the addition of $P_{M C}$. This is due to the fact that the contribution of these heating powers to the total electron heat flux are small. For the modulation amplitude, the most striking difference resides in the rather good match between modelling and experiment for $\Delta t 2$ at $20 \mathrm{~Hz}$, but about $50 \%$ lower than data for $\Delta t 1$, whereas at $8 \mathrm{~Hz}$, the modelled amplitude is too low by a factor of 2 to 3 . Independently of the comparison with the data, it should be noted that the amplitude profiles yielded by the modelling depend only weakly on the four assumptions. At $f_{\text {mod }}=8 \mathrm{~Hz}$, the differences are very small, at $20 \mathrm{~Hz}$, the increase of the amplitude for 1.5P $P_{e, \text { direct }}$ appears clearly. The influence of $P_{M C}$ is visible but weak. Therefore, none of the cases can be favoured on hand of the amplitude profile.

In contrast, the phase profiles are more sensitive to the assumptions and therefore provide more information to assess their validity. It should first be underlined that the phase delay in the central region, $R<3.2 \mathrm{~m}$, results from the combination of $P_{e, \text { direct }}$ and $P_{e, \text { coll }}$ with respectively zero and long time constant and different radial profiles. Therefore, if the modulation induced by $P_{e, \text { direct }}$ is too large, the phase delay in the central region may be too small and if that due to $P_{e, \text { coll }}$ is too large, the phase delay may be too large. The modelled phase delay in the central region has about the correct value which suggests that the calculated power modulations and phase lag of $P_{e, \text { coll }}$ are realistic. For the initial case $1.0 P_{e, \text { direct }}^{\text {SELFO }}$ and $P_{M C}=0$, the agreement of the phase delay is rather poor, only the overall slope agrees roughly with the experiment. Using $1.5 P_{e, \text { direct }}^{S E L F O}$ reduces the phase in the central part because this heating power has no time delay and this yields a somewhat better overall agreement. As already mentioned above, multiplying $P_{e, d i r e c t}^{S E L F O}$ by 1.5 is overall in better agreement with the experiment. This trend will be confirmed in the remaining of this section. As expected, the non-monotonic shape is not reproduced. Adding the modulated $P_{M C}$, with average power of only of $0.2 \mathrm{MW}$ and $0.15 \mathrm{MW}$ for the $8 \mathrm{~Hz}$ and $20 \mathrm{~Hz}$ cases respectively, brings the phase delay at mid-radius very close to the experimental data. The value of the $P_{M C}$ power has been adjusted to achieve this match of the phase. The phase profile is very flat and therefore the agreement with the data much better than without $P_{M C}$, but the non-monotonic character, expected here at $R \approx 3.45 \mathrm{~m}$, is not reproduced at $8 \mathrm{~Hz}$ and only weakly at $20 \mathrm{~Hz}$. Here please compare modelling to data for $\Delta t 1$ at $8 \mathrm{~Hz}$ and $\Delta t 2$ at $20 \mathrm{~Hz}$. This is due to the high stiffness: the fast propagation of the induced perturbation does not reflect the localised deposition of the heating power. Similar results are obtained for the other 2 time intervals with $P_{M C}$ located at $R \approx 3.3 \mathrm{~m}$ (not shown). 
The discrepancy between experimental amplitudes and the too low modelled ones at $8 \mathrm{~Hz}$ has been tested in simulations where we increased the amplitude of the power modulation. There are three possibilities: the modulation of $P_{e, \text { direct }}$ is too small, or that of $P_{e, \text { coll }}$, or both are too small. This yielded the following results. The need for a larger power modulation of $P_{e, \text { direct }}$ requires $P_{e, \text { direct }}$ itself to be correspondingly larger, because for direct heating the amplitude of the power modulation is directly proportional to the time-averaged power. The amplitude exhibits then a better agreement, but, as this power is injected with zero phase, the simulated phase delay decreases and becomes clearly too small compared to the experimental points. This hypothesis is therefore not realistic. Alternatively, one can assume that the modulation of $P_{e, \text { coll }}$ is larger than predicted by the calculations. This increases the modulation contribution with long time scale and therefore the phase delay which becomes too large. Therefore, assuming the modulation of $P_{e, \text { coll }}$ being larger implies that the time constant should simultaneously decrease which seems in contradiction with an increase of collisional heating power provided by RF-accelerated ions. Consequently, only an adequate increase of the modulation amplitude of both $P_{e, \text { direct }}$ and $P_{e, \text { coll }}$ would allow to increase the amplitude while keeping the phase delay at the same value, which does not seem to be explained by simply changing the ${ }^{3} \mathrm{He}$ concentration. Therefore, under the assumption of $\chi_{e, s}=1$, we could not find a realistic assumption which would provide a good match of both amplitude and phase at the 2 frequencies.

The results for the low stiffness case, $\left(\chi_{e, s}=0.1, R / L_{T_{e, c r i t}}=4.8\right)$, are shown Fig. 31 . Here also, the match of the $T_{e}$ profile is perfect and does not vary for the different cases. The overall features are very similar to those exhibited for the high stiffness case. The modelled amplitude is somewhat higher than for $\chi_{e, s}=1.0$, as expected for lower stiffness, but still lower than the data at $8 \mathrm{~Hz}$. The effect of $P_{M C}$ are stronger than for $\chi_{e, s}=1$ which is in agreement for transport with lower stiffness: in fact, due to the slower propagation of the perturbation, the modulation profiles reflect more clearly localised heating powers. This is particularly well illustrated by the phase profiles. Whereas for $P_{M C}=0$ the agreement is very poor, the slope is too steep as expected for low stiffness, a very good agreement of the phase can be achieved with modulated $P_{M C}$ set at the required radial position for $\Delta t 1$ at $8 \mathrm{~Hz}$ and $\Delta t 2$ at $20 \mathrm{~Hz}$, with respective averaged power of $0.2 \mathrm{MW}$ and $0.15 \mathrm{MW}$. The non-monotonic shape of the phase is well reproduced for both frequencies, a bit more accurately at $20 \mathrm{~Hz}$ because at higher $f_{\text {mod }}$ the effect remains more localised. Setting the radial position of $P_{M C}$ at the value 

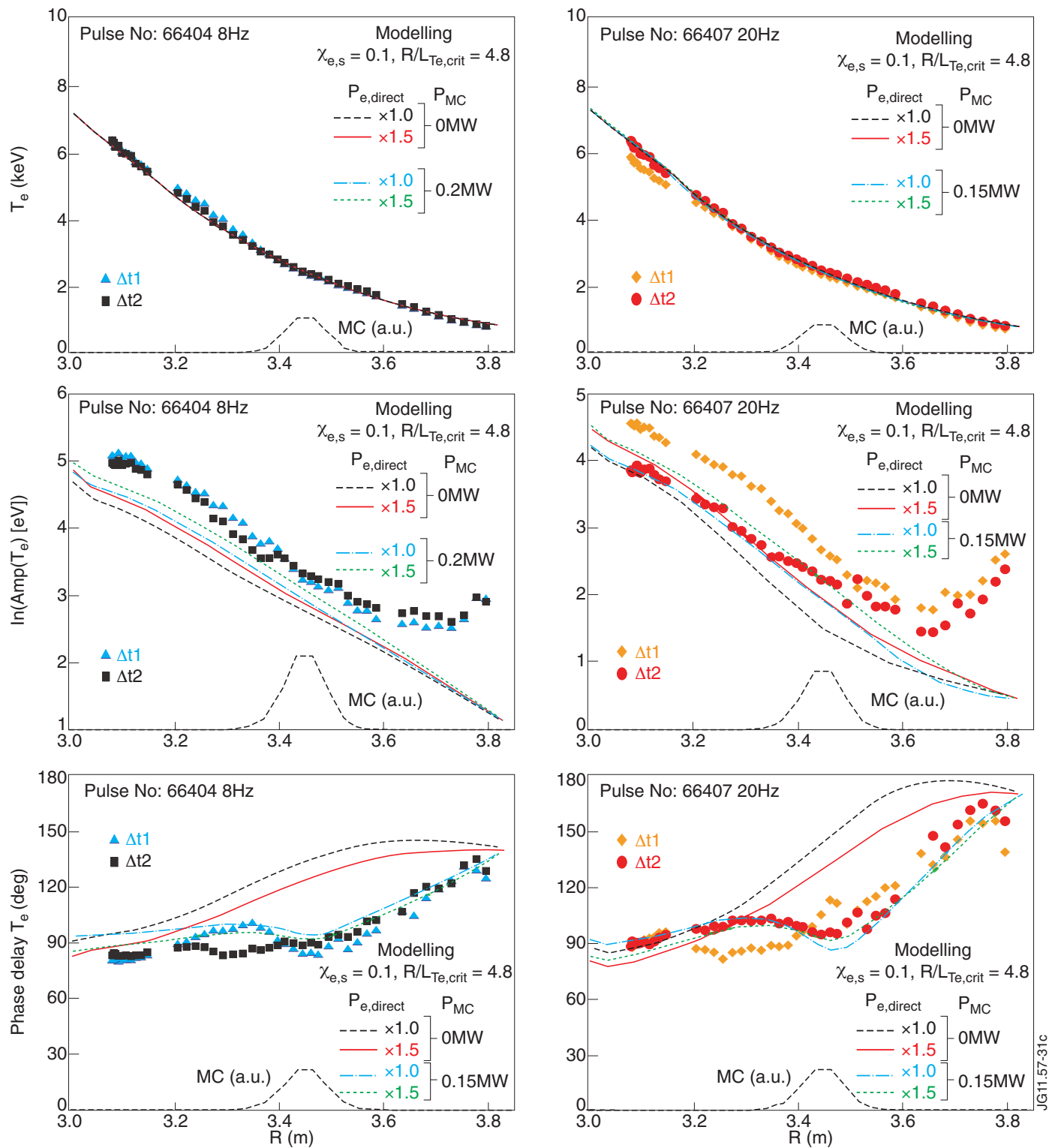

Figure 31. Time-averaged $T_{e}$, amplitude and phase of the $T_{e}$ modulation at $8 \mathrm{~Hz}$ (left panels) and $20 \mathrm{~Hz}$ (right panels). Symbols are experimental data with same coding as in Fig. 29. Modelling using pure diffusion with $\chi_{e}$ only, low stiffness cases $\chi_{e, s}=0.1$ and different assumptions on $P_{e, \text { direct }}$ and off-axis $P_{M C}$ : line types defined in legend.

corresponding to the other time interval also yields good results (not shown). The hypothesis $1.5 P_{e, \text { direct }}^{S E L F O}$ yields somewhat better results in the centre at $8 \mathrm{~Hz}$, whereas the differences are within the error bars at $20 \mathrm{~Hz}$. Finally, it should be underlined that the square modulation of $P_{M C}$ excites a $T_{e}$ modulation at the $3^{r d}$ harmonic which, according to the simulations, reaches $1-2 \mathrm{eV}$ at the deposition of $P_{M C}$ and is just buried in the noise of the measurement which has the same magnitude. This indicates that $P_{M C}$, if it exists, is not much larger than that 
required by the match of the simulation to the experiment, otherwise the $3^{\text {rd }}$ harmonic would be detected in the experimental data, which is not the case.

In conclusion, assuming that the electron heat transport is purely diffusive and that it follows the critical gradient model, as done in earlier studies, the amplitude is significantly too small, in particular at $8 \mathrm{~Hz}$ which is clearly outside of the uncertainties on the power deposition calculations. The very flat and even non-monotonic shape of the phase delay implies the existence of modulated electron heating power deposited off-axis, with zero phase delay. The required averaged power lies between 0.1 MW and 0.2 MW which is small compared to the total heating power and within the range predicted by TORIC for mode conversion under our experimental conditions. The modelling clearly indicates that, in the frame of this hypothesis, electron heat transport as described by the CGM must have a low stiffness, which is compatible with the previous JET results with dominant electron heating [40].

\subsection{Modelling of electron heat transport with ITG-induced off-diagonal contribution}

In this subsection we take into account the contribution of ITG to electron heat transport which is predicted by the GYRO calculations. This situation is complex because the electron heat transport is then also strongly influenced by the ion channel through the ITG off-diagonal term. This affects both time-average and modulation of $T_{e}$. Therefore, the ion transport must be simulated well enough to ensure a realistic modelling of the electron channel. For electron heat transport we take into account both $\chi_{e}$ and $\chi_{i e}$ in the simulation, according to Eqs. $3(j=e)$ and 4 respectively. The values of the coefficients, derived from the GYRO calculations, are $\left(\chi_{e, s}=0.05, \alpha_{e}=2.5\right.$ with $\left.R / L_{T_{e, c r i t}}=7\right)$ for $\chi_{e}$ of the TEM (Fig. 10 panel 4) and those listed in Table 2 for $\chi_{i e}$. For this channel, low values of $\alpha_{i e}$, as those of the two upper rows of Table 2, induce a very strong contribution to electron heat transport just above the threshold which then saturates for larger values of $R / L_{T_{i}}$. Using in the modelling $\alpha_{i e}=0.12$, yielded by the free fit on the GYRO points without any off-set correction linked to the TEM, yields extremely poor agreement with the experiment for the time-averaged $T_{e}$, which is much too low in the centre, and for the modulation data as well. Much better results are obtained with values of $\alpha_{i e}$ corresponding to the upper range of those listed in Table 2. In fact, good modelling results are achieved for $\alpha_{i e} \approx 0.5$, i.e. the same dependence as for ion transport. As $\chi_{i}$ and $\chi_{i e}$ are both driven by the ITG instability, this is a logical hypothesis and we also assume the same threshold. In the modelling of electron transport in the presence of 
the off-diagonal term, the ion transport must be properly simulated. Therefore, the simulations were carried out with some of the best cases for ion transport presented in the above section. These are: i) $\chi_{i, s}=1.1$ flat and $R / L_{T_{i, c r i t}}=3.2$ flat; ii) $\chi_{i, s}=1.1$ flat and $R / L_{T_{i, c r i t}}$ peaked with nominal value 3.2 ; iii) $\chi_{i, s}$ hollow with nominal value 2.2 and $R / L_{T_{i, c r i t}}=3.2$ flat. For the electron transport simulations, three values of $\chi_{i e, s}$ are used: $0.8,0.6,0.4$. These are lower than that yielded by GYRO, but we also use for $\chi_{i, s}$ a lower value, 1.1 instead of 2.25. For the hollow $\chi_{i, s}$ case, $\chi_{i e, s}$ is also hollow with the same shape. The TEM contribution, which is small in the core as shown above, is kept fixed and determined by the coefficients yielded by the GYRO results. The modulated heat sources are $P_{e, \text { coll }}^{S E L O}$ and $1.5 P_{e, \text { direct }}^{S E L F O}$ while we first assume $P_{M C}=0$. Here also, multiplying $P_{e, d i r e c t}^{S E L F O}$ by 1.5 yields better results. For all the simulations, the results for the ions are of course the same as those presented in the previous section and we do not show them again.

6.3.1. Assuming flat $\chi_{i, s}$ and $R / L_{T_{i, c r i t}}$ profiles: The modelling results for the electron channel for the first case, $\chi_{i, s}=1.1$ and flat $R / L_{T_{i, c r i t}}$, are shown in Fig. 32 for $8 \mathrm{~Hz}$ and $20 \mathrm{~Hz}$. The time-averaged $T_{e}$ profile is in good agreement for the lowest ITG-induced transport, $\chi_{i e, s}=0.4$, whereas electron heat transport is obviously too high for the values 0.6 and 0.8 . The $T_{e}$ modulation amplitude agrees very well with the data at $8 \mathrm{~Hz}$, which is in strong contrast to the cases assuming pure diffusion, see Figs. 30 or 31 . At $20 \mathrm{~Hz}$, the agreement is less good than at $8 \mathrm{~Hz}$, in particular the slope is too steep, which is comparable to the cases with diffusion only. As for the pure diffusion cases, the agreement of the phase delay in the centre is rather good and depends weakly on $\chi_{i e, s}$, but further out the slope is much steeper than the experimental data. In the central region, the phase delay exhibits a non-monotonic behaviour which is roughly comparable to the experimental data. This is particularly clear at $8 \mathrm{~Hz}$ for $\chi_{i e, s}=0.6$, to be compared to the data of interval $\Delta t 2$.

This non-monotonic behaviour is caused by the modulation of the ion heating which acts on $T_{e}$ through $\chi_{i e}$ as follows. The off-diagonal term $\chi_{i e}$ is modulated by $R / L_{T_{i}}$. This means that, during each modulation cycle, the electron heat transport driven by $\chi_{i e}$ increases and decreases in phase with $R / L_{T_{i}}$. It is well-known, [41, 42], that, at constant heating power, a modulation of heat transport, here through $\chi_{i e}$, induces a modulation of the temperature which is such that the temperature increases when transport decreases and vice-versa. In our situation, the $R / L_{T_{i}}$ modulation induces a $T_{e}$ modulation which is out of phase and has the 

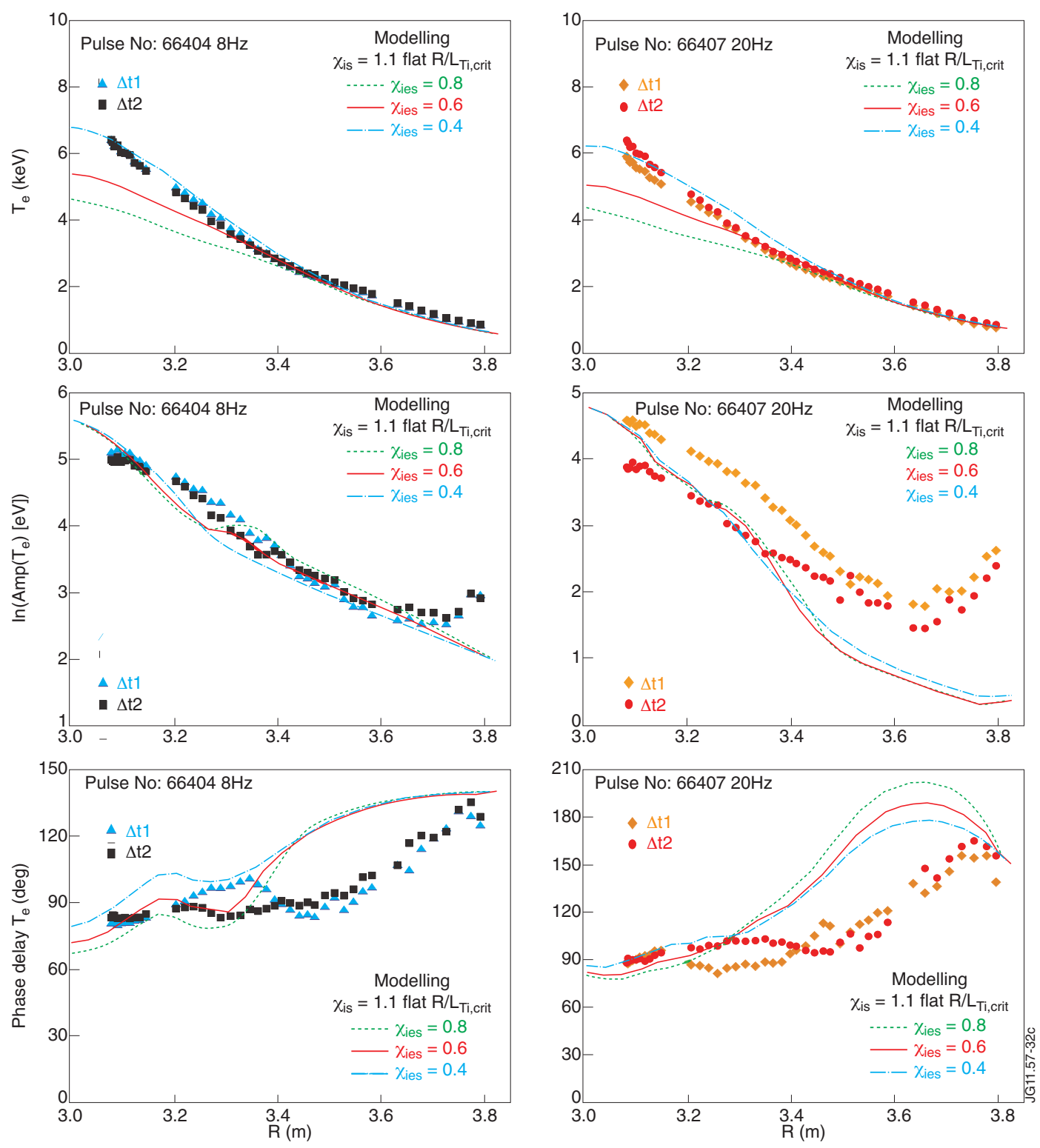

Figure 32. Time-averaged $T_{e}$, amplitude and phase of the $T_{e}$ modulation at 8 and 20 Hz. Modelling with ITG contribution $\chi_{i e}$ for ion transport parameters $\chi_{i, s}=1.1$ and flat threshold $R / L_{T_{i, c r i t}}=3.2$.

profile of the modulation of $\chi_{i e}$.

This is illustrated in Fig. 33 by the profiles of amplitude and phase delay of $\chi_{i e}$ for the three different values of $\chi_{i e, s}$. The modulation amplitude of $\chi_{i e}$ exhibits a maximum at $R \approx 3.2$ m. It occurs where $R / L_{T_{i}}$ has the largest oscillation and this is dominated by that of $\nabla T_{i}$. It is located off-axis because the modulation of $\chi_{i e}$ close to the plasma centre is very small as $\nabla T_{i}$ tends to zero there. Note also that, logically, the amplitude of $\chi_{i e}$ increases with $\chi_{i e, s}$. The 

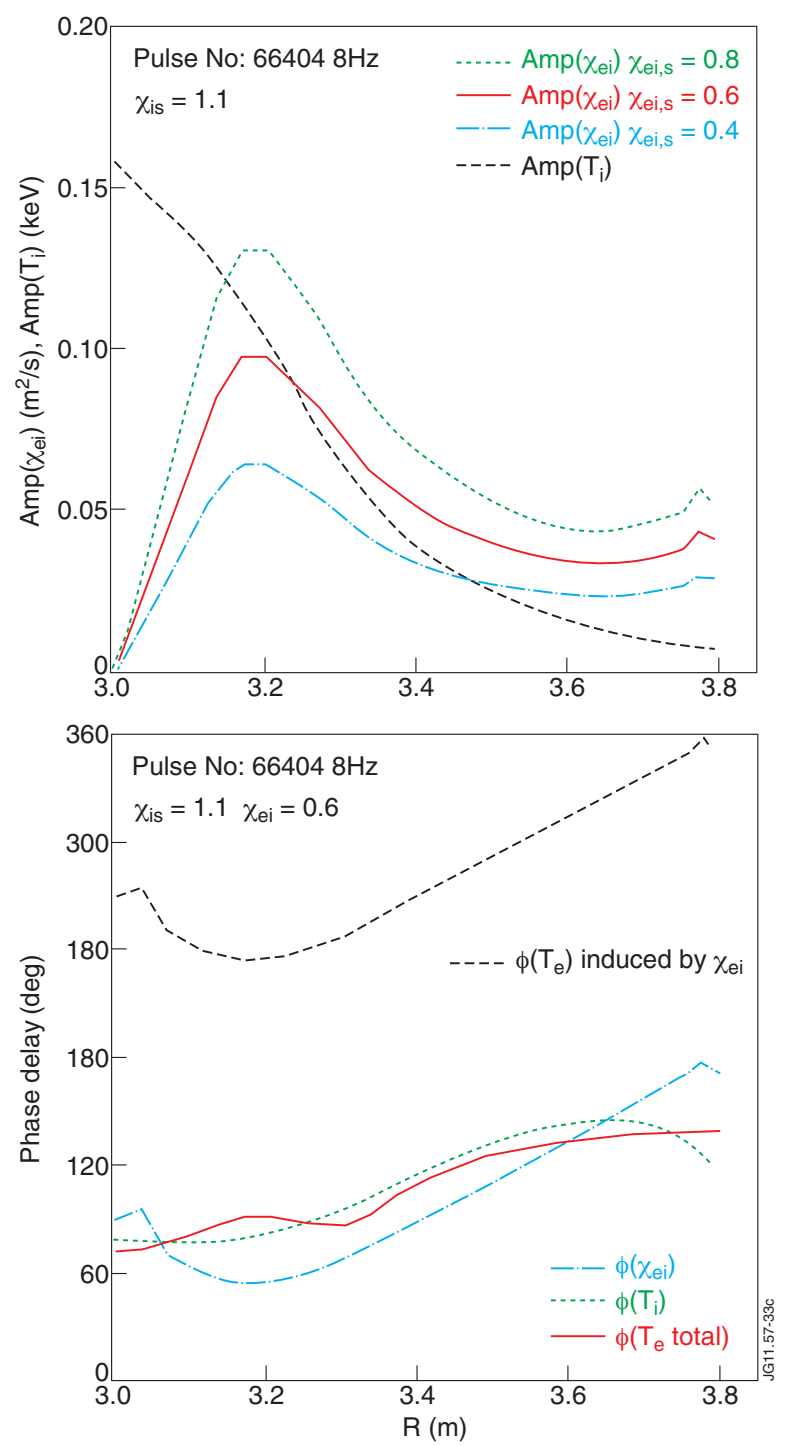

Figure 33. Modelled modulation amplitude of $\chi_{i e}$ for three values of $\chi_{i e, s}$, for the case $\chi_{i, s}=1.1$. That of $T_{i}$ is also shown to illustrate the different radial profiles. Different contributions to the phase delay of $T_{e}$, as labelled in the legend and explained in the text.

modulation amplitude of $\chi_{i e}$ at its maximum reaches about $10 \%$ of the time averaged value of $\chi_{i e}$ at this location. For comparison, we also plotted in Fig. 33 the amplitude of the $T_{i}$ modulation which depends on $\chi_{i}$ only and is therefore the same for all three values of $\chi_{i e, s}$. The profiles of the different contributions to the phase delay of $T_{e}$ due to the $\chi_{i e}$ modulation are also plotted in Fig. 33. They weakly depend on the values of $\chi_{i e, s}$ and are therefore plotted here for $\chi_{i e, s}=0.6$ only for clarity. The phase delay of the $\chi_{i e}$ modulation is somewhat smaller than that of $T_{i}$ because $\nabla T_{i}$ reacts faster to a perturbation than $T_{i}$ itself. The phase of the $T_{e}$ 
modulation resulting from the $\chi_{i e}$ modulation and out of phase compared to it is also plotted. The modulation of $T_{e}$ results from the interference of three "heat waves": the two initial ones excited by $P_{e, \text { coll }}$ and $P_{e, \text { direct }}$ to which that due to $\chi_{i e}$ combines. The relative amplitude of these three contributions and the phase difference between them determines the final phase delay of $T_{e}$. The non-monotonic behaviour of the phase delay of $T_{e}$ is due to the fact that the amplitude and phase profiles of the modulation induced by $\chi_{i e}$ are quite different from those of the $T_{e}$ modulation induced by $P_{e, c o l l}$ and $P_{e, \text { direct }}$. This is why, in contrast to the effect induced on the phase delay by the presence of an off-axis source $P_{M C}$, as discussed above, the position of the minimum of the $T_{e}$ phase delay, here at $R \approx 3.3 \mathrm{~m}$, does not correspond to the position of the maximum of the amplitude of the $\chi_{i e}$ modulation at $R \approx 3.2 \mathrm{~m}$. This is very schematically and only qualitatively illustrated in Fig. 34 which shows that the contribution due to the $\chi_{i e}$ modulation can reduce the phase delay compared to the situation with a perturbation induced by $P_{e, \text { coll }}$ and $P_{e, \text { direct }}$ only.

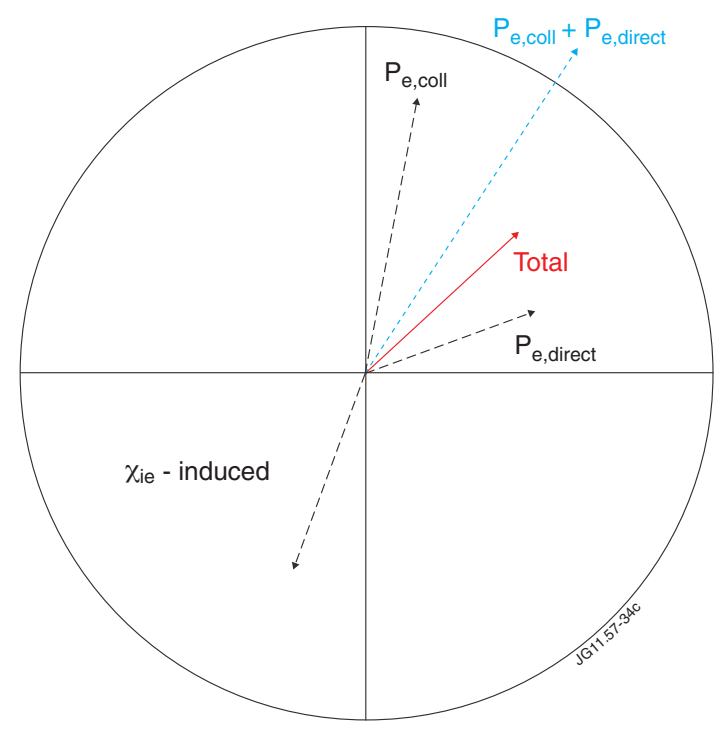

Figure 34. Polar coordinate plot showing schematically the combination of the three different contributions to the $T_{e}$ modulation.

6.3.2. Assuming flat $\chi_{i, s}$ and peaked $R / L_{T_{i, c r i t}}$ profiles: The second modelling study with offdiagonal term was performed with the case $\chi_{i, s}=1.1$ flat and $R / L_{T_{i, c r i t}}$ peaked with nominal value 3.2. Here also we considered the three values $0.4,0.6$ and 0.8 for $\chi_{i e, s}$. The results are shown in Fig. 35. The $T_{e}$ profile is well reproduced by $\chi_{i e, s}=0.6$, whereas 0.4 and 0.8 are respectively above and below the experimental data. The comparison with the previous case, 
Fig. 32, indicates that the peaked shape of $R / L_{T_{i, c r i t}}$ also influences the shape of $T_{e}$. Apart from this, the overall features of amplitude and phase delay are quite similar to those of the previous case. The amplitude is insensitive to the value of $\chi_{i e, s}$. The phase delay is sensitive to the value of $\chi_{i e, s}$ in the centre of the plasma. In agreement with the $T_{e}$ profile, the case $\chi_{i e, s}=0.6$ yields the results which are closer to the data, including a good match of the nonmonotonic part. It should be underlined that the best match is obtained with values for $\chi_{i, s}$ and $\chi_{i e, s}$ which are about two times lower than those yielded by the GYRO calculations, but that the ratio $\chi_{i, s} / \chi_{i e, s} \approx 2$, corresponds to that of the GYRO calculations.

6.3.3. Assuming hollow $\chi_{i, s}$ and flat $R / L_{T_{i, c r i t}}$ profiles: Finally we also present in Fig. 36 the results for $\chi_{i, s}=2.2$ hollow and $R / L_{T_{i, c r i t}}=3.2$ flat. In this case we also modelled with $\chi_{i e, s}=1.1$ to include the case $\chi_{i, s} / \chi_{i e, s}=2$. Overall the results are comparable to the previous ones, but somewhat poorer. The best agreement for $T_{e}$ is yielded by $\chi_{i e, s}=0.6$ or 0.8 , whereas with $\chi_{i e, s}=1.1$ transport is too high . The amplitude at $8 \mathrm{~Hz}$ is not as good as in the previous cases and at $20 \mathrm{~Hz}$ the slope is by far too steep, reminiscent of the $T_{i}$ modulation amplitude in Fig. 24. The best phase delay in the central part is yielded by $\chi_{i e, s}=1.1$, which is in disagreement with the lower value required for the $T_{e}$ profile. Here also, in the outer part of the plasma $(R>3.4 \mathrm{~m})$, the agreement is very poor at $8 \mathrm{~Hz}$, clearly better at $20 \mathrm{~Hz}$. The results yielded by this case are overall poorer than those with peaked threshold.

6.3.4. Influence of stiffness curvature $\alpha_{i e}$ : The above simulations have been carried out with the stiffness curvature coefficients $\alpha_{i}=\alpha_{i e}=0.54$. We have shown at the end of Sect. 5 that this dependence is not crucial for the $T_{i}$ simulations, Fig. 27. However, it plays a key role for the electron modulation data, as illustrated in Fig. 37 which displays amplitude and phase delay for different values of $\alpha_{i e}$ between 0.54 and 1.0, while the other parameters are kept constant.

We chose the case $\chi_{i, s}=1.1$ with peaked $R / L_{T_{i, c r i t}}$ and selected $\chi_{i e, s}=0.65$, instead of 0.6 , because the results are somewhat closer to the data. The amplitude is weakly affected by the value of $\alpha_{i e}$ and the results are similar to those of Fig. 35. The most visible variation induced by the scan of $\alpha_{i e}$ appears in the phase delay at $8 \mathrm{~Hz}$ : the non-monotonic shape changes such that the maximum is shifted outwards for large values of $\alpha_{i e}$ while the depth of the oscillation decreases and better matches the data. Note that the outer part of the profile is also closer to the data as $\alpha_{i e}$ increases. For $\alpha_{i e}=0.9$, the modelled phase delay at $8 \mathrm{~Hz}$ agrees 

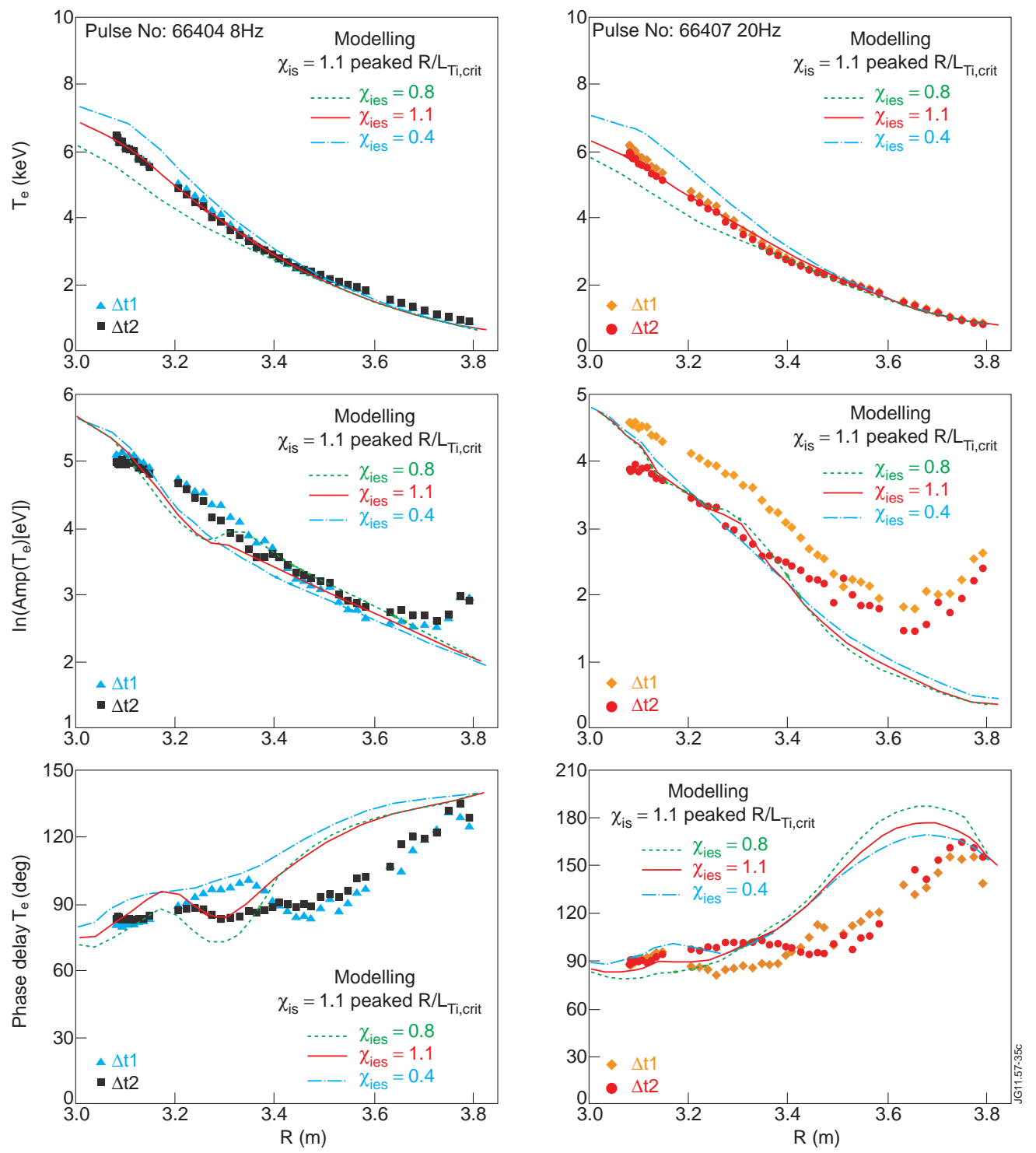

Figure 35. Time-averaged $T_{e}$, amplitude and phase of the $T_{e}$ modulation at 8 and 20 Hz. Modelling with ITG contribution $\chi_{i e}$ for ion transport parameters $\chi_{i, s}=1.1$ and peaked threshold $R / L_{T_{i, \text { crit }}}$ nominal value 3.2.

well with the data of interval $\Delta t 2$. However, we could not match the phase delay for $\Delta t 1$ in the central plasma. At $20 \mathrm{~Hz}$, the phase delay is well matched for $R<3.45 \mathrm{~m}$, but as previously the slope is too steep further outside. Therefore, a perfect match cannot be reached, but this scan illustrates how sensitive the modulated electron data are with respect to the dependences of $\chi_{i e}$, in particular in the central region. This is due to the slope of $\chi_{i e}$ with respect to $R / L_{T_{i}}$. For low values of $\alpha_{i e}$, this slope is large for low values of $R / L_{T_{i}}-R / L_{T_{i, \text { crit }}}$ and therefore electron heat transport driven by $\chi_{i e}$ very sensitive to the $T_{i}$ modulation where $R / L_{T_{i}}$ is close 

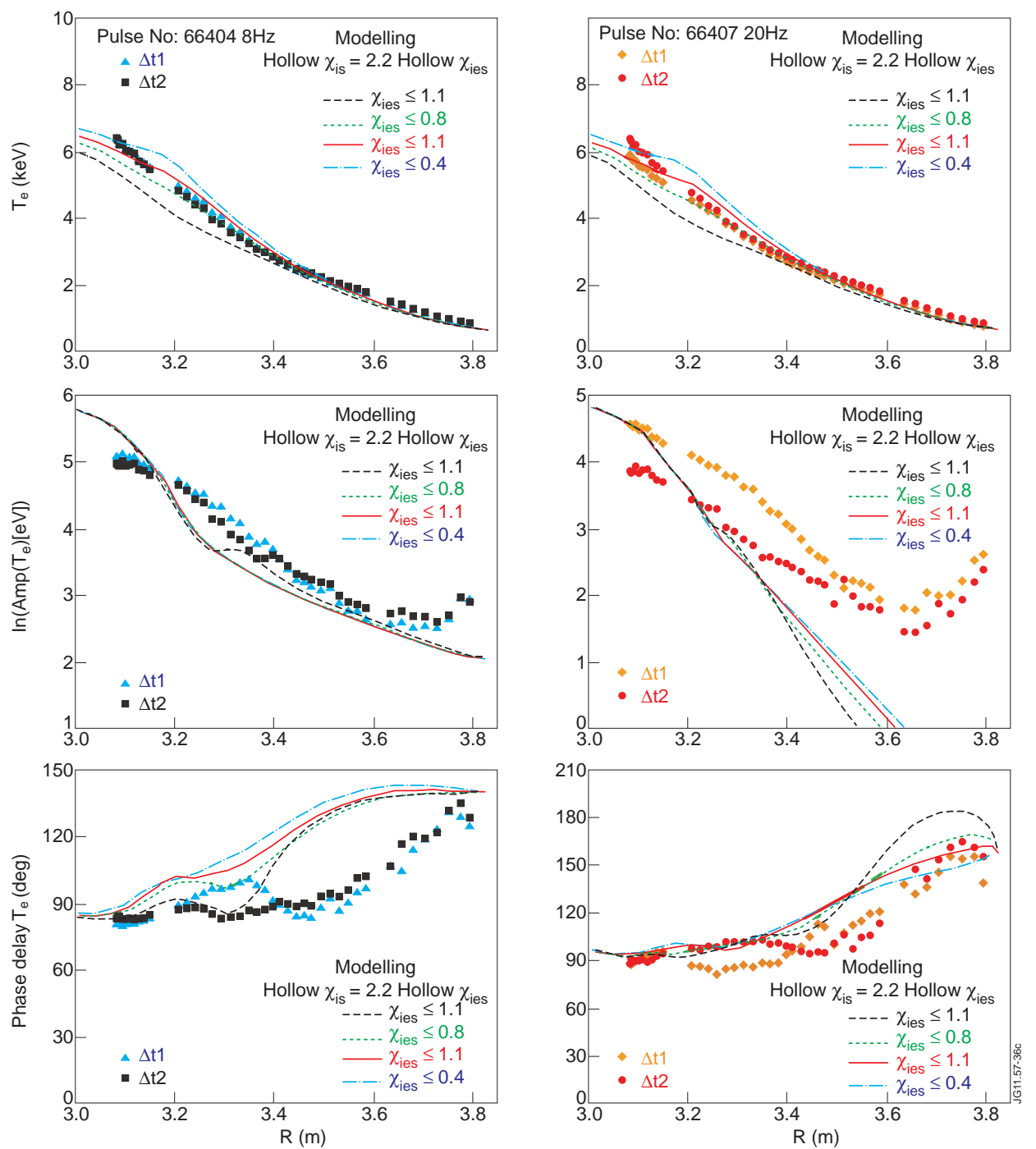

Figure 36. Time-averaged $T_{e}$, amplitude and phase of the $T_{e}$ modulation at 8 and 20 $H z$. Modelling with ITG contribution $\chi_{i e}$ for ion transport using hollow $\chi_{i, s}$ profile, nominal value 2.2 , and flat threshold $R / L_{T_{i, c r i t}}=3.2$.

to $R / L_{T_{i, c r i t}}$, i.e. in the central plasma. As $\alpha_{i e}$ increases, the sensitivity decreases in the plasma centre but increases further outside.

This is illustrated in Fig. 38 which shows the amplitude of the modulation of $\chi_{i e}$, reminding that this quantity contributes to the $T_{e}$ modulation. The phase of $\chi_{i e}$ is almost not influenced and therefore not shown. The shift of the maximum of $\chi_{i e}$ modulation away from the plasma and the change of the profile shape with increasing $\alpha_{i e}$ are clearly seen. The reduction of the amplitude in the plasma centre and its increase at larger radii for increasing values of $\alpha_{i e}$ are the reasons for the phase behaviour of the $T_{e}$ modulation in the $\alpha_{i e}$ scan. 

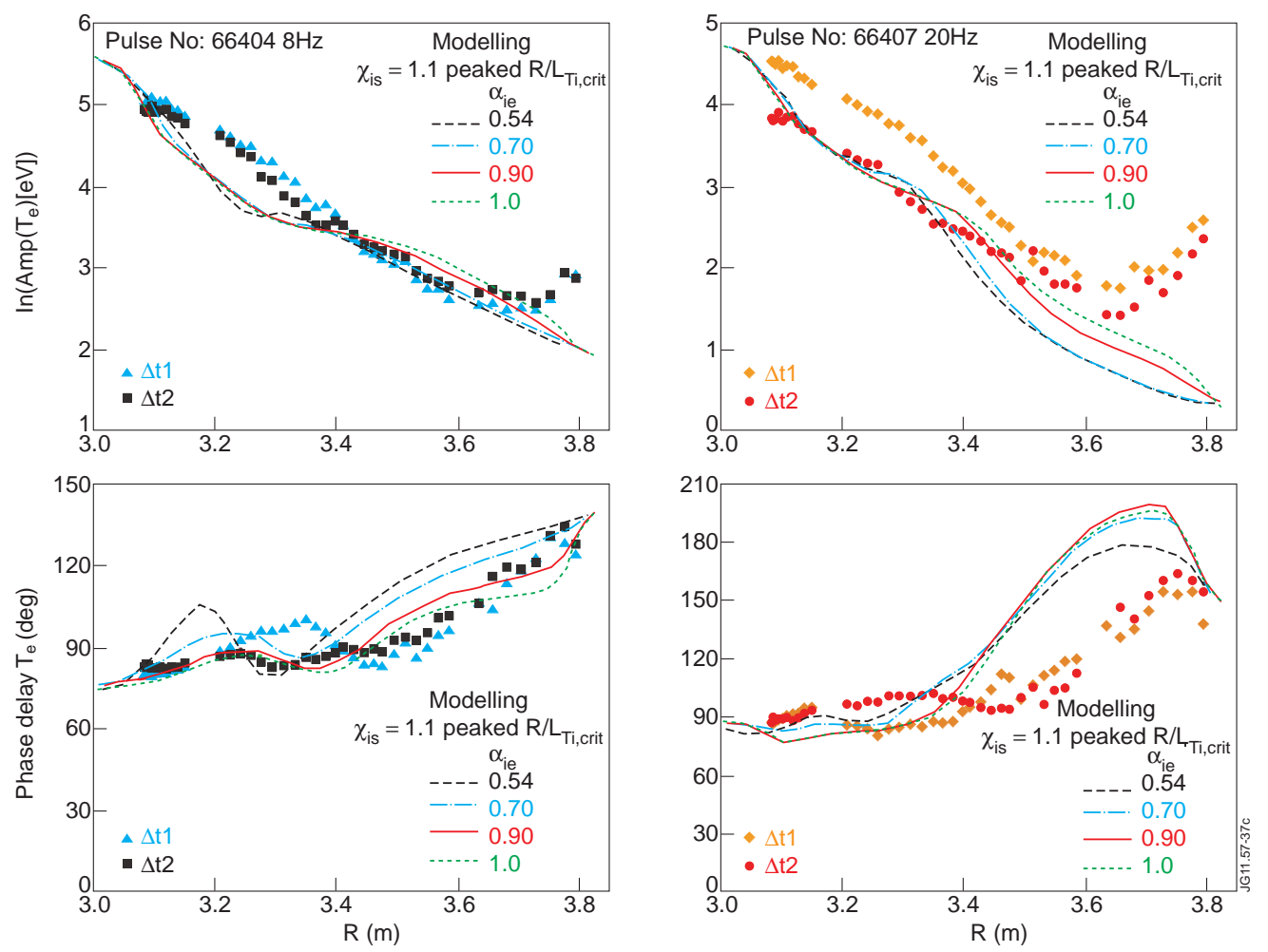

Figure 37. Amplitude and phase of the $T_{e}$ modulation at 8 and $20 \mathrm{~Hz}$. Modelling as case $\chi_{i e, s}=0.65$ and scan of $\alpha_{i e}$ Ion transport parameters are $\chi_{i, s}=1.1$ and peaked threshold $R / L_{T_{i, c r i t}}$ nominal value 3.2.

In summary, for three different hypotheses on ion heat transport, the simulations for electron heat transport including the ITG-induced contribution exhibit similar features. They all yield better results than the simulations which do not take this contribution into account. However, none of the cases yields really satisfactory results for all three quantities, $T_{e}$ profile, amplitude and phase delay, at the two frequencies. The case with peaked $R / L_{T_{i, \text { crit }}}=3.2$ and $\chi_{i, s}=1.1$ may be considered as overall the best, but the amplitude profile at $20 \mathrm{~Hz}$ is too steep, as well as the phase profile in the outer region, for the two frequencies.

6.3.5. Adding off-axis mode conversion power deposition: The poor match of the phase delay in the outer part of the plasma, in particular at $20 \mathrm{~Hz}$, suggests to consider, as above, the possibility of off-axis electron heating, $P_{M C}$. We investigate this hypothesis for the best cases of the above Figs. 35 and 37: $\left(\chi_{i e, s}=0.6 ; \alpha_{i e}=0.54\right)$ and $\left(\chi_{i e, s}=0.65 ; \alpha_{i e}=0.90\right)$, while for the ions we keep $\chi_{i, s}=1.1$, peaked $R / L_{T_{i, c r i t}}=3.2$. As above, the value of $P_{M C}$ has been adjusted to match the phase delay at the off-axis position of its deposition. For the first case, 

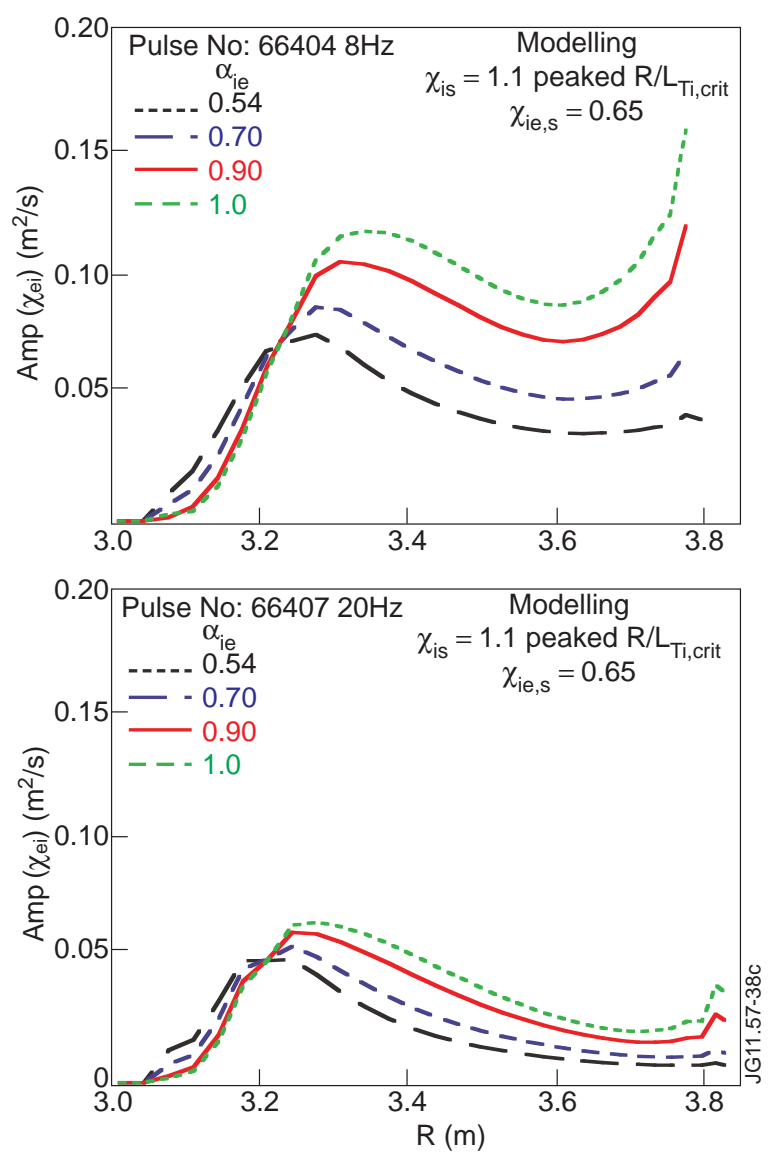

Figure 38. Amplitude of the $\chi_{i e}$ modulation at 8 and $20 \mathrm{~Hz}$. Modelling as case $\chi_{i e, s}=0.65$ and scan of $\alpha_{i e}$ Ion transport parameters are $\chi_{i, s}=1.1$ and peaked threshold $R / L_{T_{i, c r i t}}$ nominal value 3.2.

the results are illustrated in Fig. 39. Here also, low values of $P_{M C}, 0.1 \mathrm{MW}$ at $8 \mathrm{~Hz}$ and 0.075 MW at $20 \mathrm{~Hz}$, are sufficient for good agreement. The $T_{e}$ profile is not affected and therefore not shown. The amplitude is quite good for both frequencies. Whereas the presence of $P_{M C}$ has almost no influence on the amplitude at $8 \mathrm{~Hz}$, we underline its very positive effect at $20 \mathrm{~Hz}$ due to the low amplitude at this frequency and therefore high sensitivity to additional power. The phase delay can also be very well reproduced for both frequencies. At $8 \mathrm{~Hz}$ the phase delay for $\Delta t 2$ is well matched, but we could not match that of $\Delta t 1$. The $20 \mathrm{~Hz}$ case shows very convincingly that the good agreement for amplitude and phase is achieved simultaneously for the same time interval, here $\Delta t 2$.

None of the simulations presented above is able to reproduce the higher $T_{e}$ modulation amplitude of the the time interval $\Delta t 1$ at $20 \mathrm{~Hz}$ and its corresponding very flat phase profile in the centre up to $R \approx 3.35 \mathrm{~m}$. A good match of both amplitude and phase delay can be 

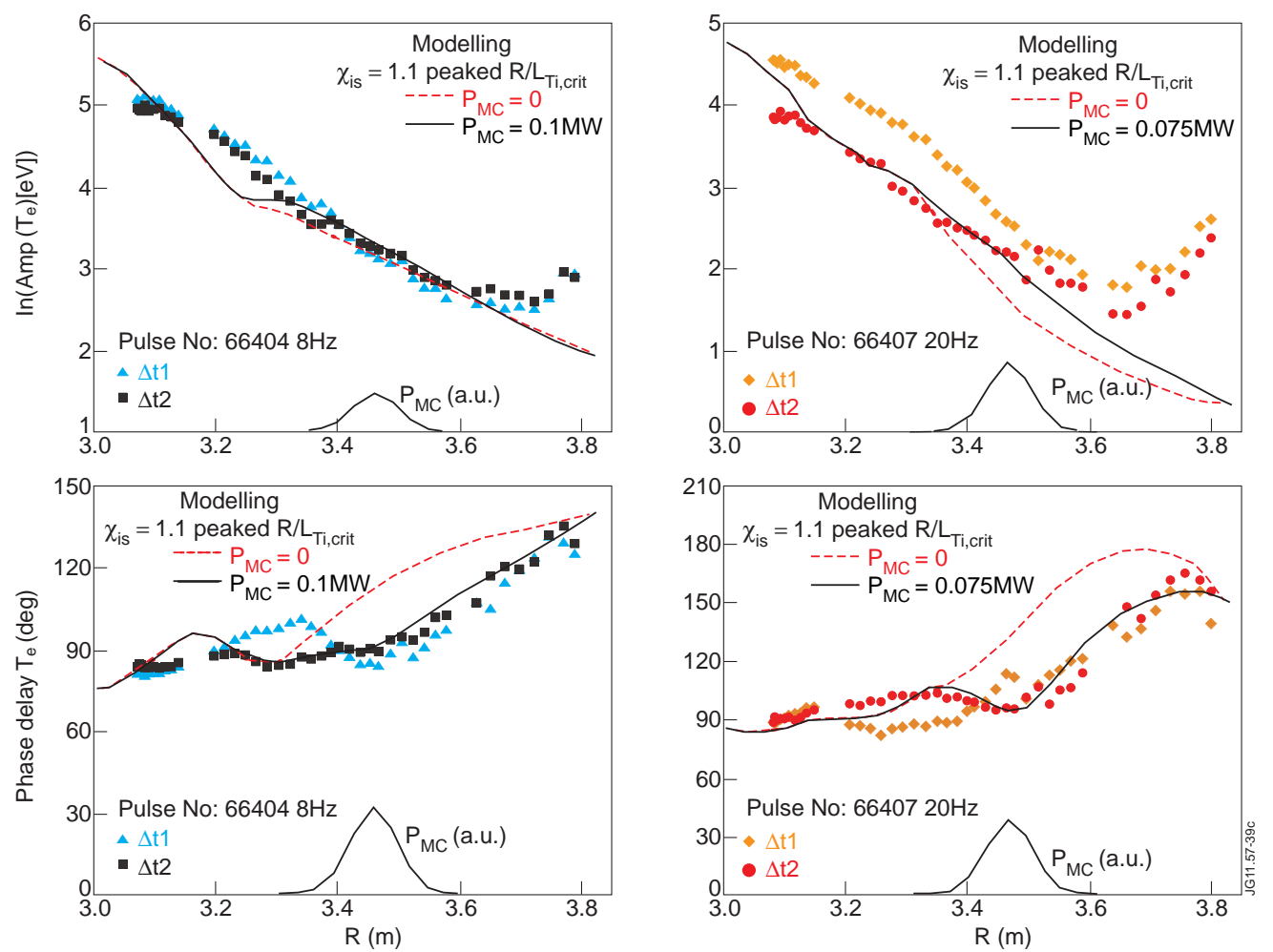

Figure 39. Time-averaged $T_{e}$, amplitude and phase of the $T_{e}$ modulation at 8 and $20 \mathrm{~Hz}$. Modelling with $\chi_{i e, s}=0.6 \alpha_{i e}=0.54$ with additional $P_{M C}$ at $R \approx 3.45$. Ion transport parameters are $\chi_{i, s}=1.1$ and peaked threshold $R / L_{T_{i, \text { crit }}}$ nominal value 3.2.

achieved with $P_{M C}=0.25 \mathrm{MW}$ deposited at $R \approx 3.25 \mathrm{~m}$ with a broad profile, Fig. 40 . It is worth underlying that amplitude and phase are reproduced simultaneously with good accuracy under this assumption, a convincing argument supporting the hypothesis of electron heating being deposited there with about zero phase delay. In complement, a good match of the amplitude in the very centre requires $P_{e, \text { direct }}$ to be higher, $2 \times P_{e, \text { direct }}^{S E L F O}$, which does not affect the phase. A higher value of $P_{M C}$ might imply a reduction of $P_{e, c o l l}$, represented here by $0.75 \times P_{e, \text { coll }}$ and indeed compatible with the data, including the time-averaged $T_{e}$ profile.

Finally, modelling of the cases with $\alpha_{i e}=0.9$ including off-axis $P_{M C}$ to improve the phase profile in the outer part of the plasma is presented in Fig. 41. The quality of the results is similar to the above cases. The amplitude profiles are not as good as the previous ones. A good match of the phase profiles in the central part of the plasma is obtained, as expected from the scan of $\alpha_{i e}$. However, in the outer part, the agreement is good at $8 \mathrm{~Hz}$, but poorer at 20 Hz. In particular, at $20 \mathrm{~Hz}$, the best match of the amplitude is obtained for $\Delta t 2$, but the phase delay for $\Delta t 1$ is not consistent. Here also, the time interval $\Delta t 1$ at $8 \mathrm{~Hz}$ cannot be matched. 

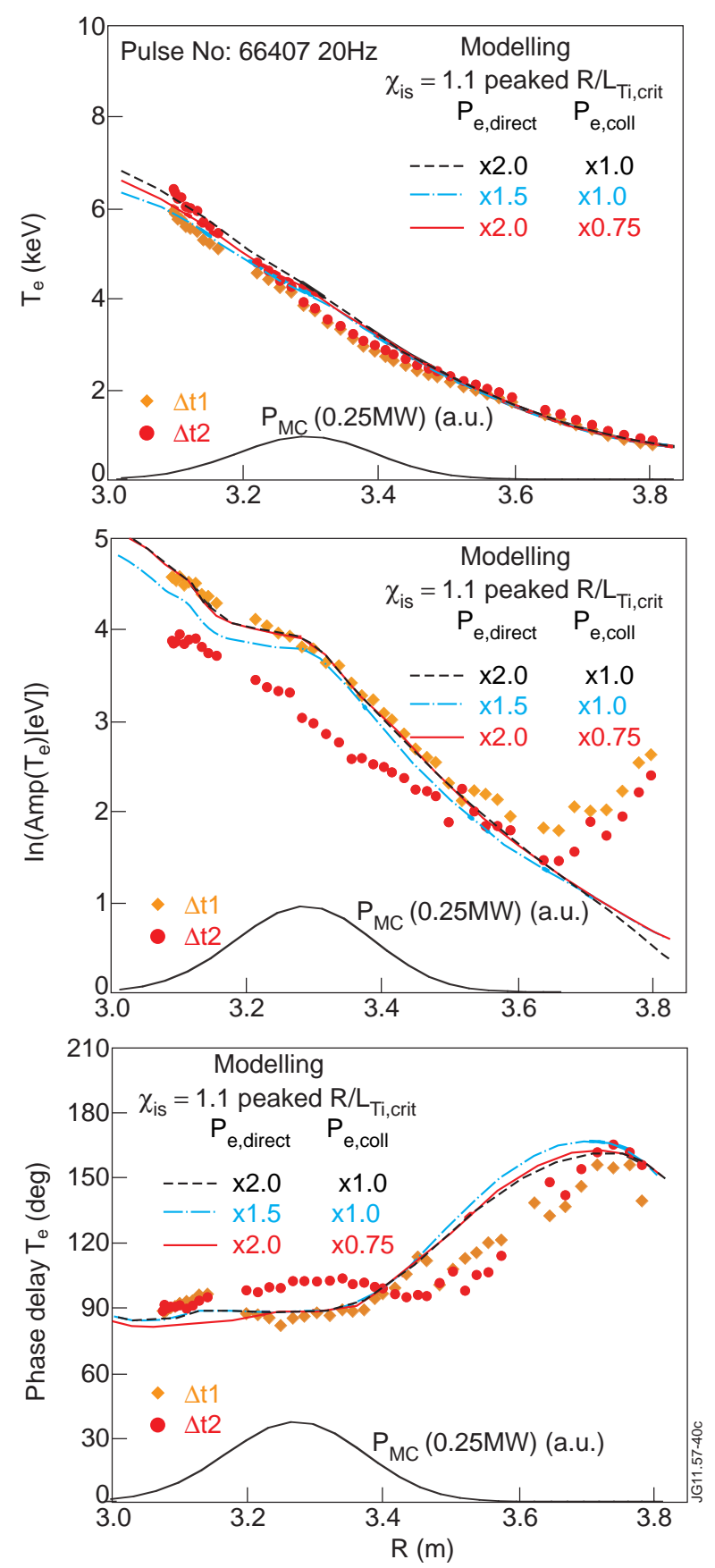

Figure 40. Time-averaged $T_{e}$, amplitude and phase of the $T_{e}$ modulation at $20 \mathrm{~Hz}$. Modelling with $\chi_{i e, s}=0.6 \alpha_{i e}=0.54$ as in Fig. 39 but with $P_{M C}=0.25 \mathrm{MW}$ at $R \approx 3.3 \mathrm{~m}$. Ion transport parameters are $\chi_{i, s}=1.1$ and peaked threshold $R / L_{T_{i, \text { crit }}}$ nominal value 3.2 .

6.3.6. Summary of the electron heat transport results: Summarising, the interpretation of electron heat transport in these experiments is complex due to the different heat sources described above. Heat transport can occur through diffusion and off-diagonal term driven 

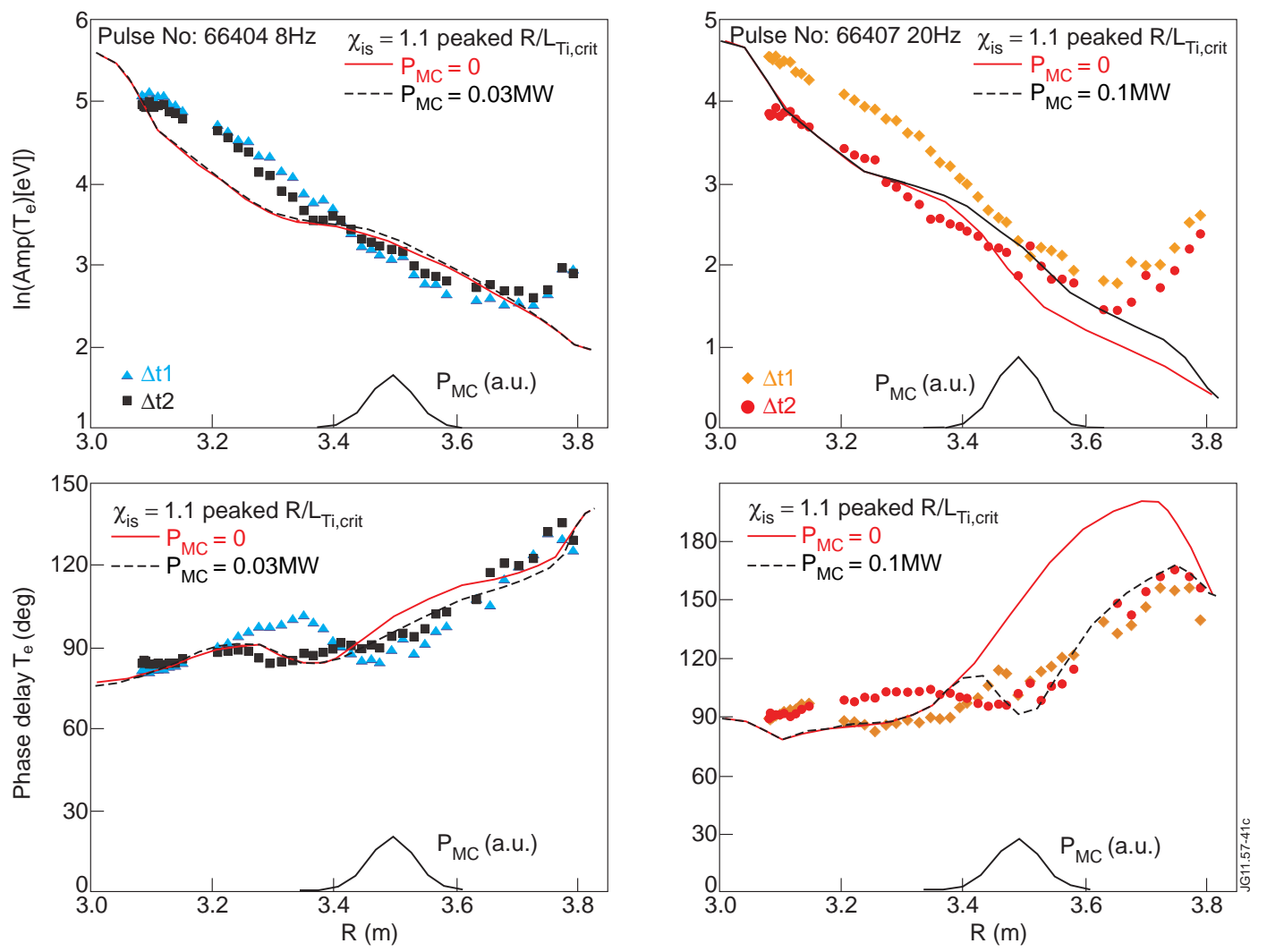

Figure 41. Amplitude and phase of the $T_{e}$ modulation at 8 and $20 \mathrm{~Hz}$. Modelling with $\chi_{i e, s}=0.65$ and $\alpha_{i e}=0.9$ as in Fig. 37 but with additional $P_{M C}$ at $R \approx 3.45$. Ion transport parameters are $\chi_{i, s}=1.1$ and peaked threshold $R / L_{T_{i, c r i t}}$ nominal value 3.2.

by the ion turbulence.

The modelling including only diffusion with an adequate choice of stiffness and threshold yields good match of the experimental time-averaged $T_{e}$ profile. However, for the modulation data in the case $P_{M C}=0$, while the amplitude at $20 \mathrm{~Hz}$ matches the data of the time interval $\Delta t 2$ quite well, the amplitude at $8 \mathrm{~Hz}$ is much too low, as well as that at $20 \mathrm{~Hz}$ compared to the $\Delta t 1$ data, in addition the phase delays at 8 and $20 \mathrm{~Hz}$ are not correctly reproduced. It is important to underline that even a high stiffness does not reproduce the flat phase profile in the centre. Adding off-axis modulated power, $P_{M C}$, yields good phase profiles at low stiffness, but poorer at high stiffness. The amplitude at $8 \mathrm{~Hz}$ remains too low, whereas the amplitude at $20 \mathrm{~Hz}$ provides the same results as in the case $P_{M C}=0$. Overall, modelling of electron heat transport assuming diffusion (diagonal term) only, yields poor results.

In contrast, including the contribution by the ITG non-diagonal contribution, as suggested by the GYRO calculations, yields an overall better agreement with the experiment. In particular, 
the modulation amplitude, even at $8 \mathrm{~Hz}$, reaches the correct magnitude and the phase profiles in the central part of the plasma are flat enough and tend to exhibit the experimentally observed non-monotonic behaviour, even with $P_{M C}=0$. The $T_{e}$ profile can be well reproduced, whereby the agreement depends on the hypothesis for ion transport. It is worth underlying that the ion channel parameters yielding a good match of the $T_{i}$ profile are necessary for a good match of the $T_{e}$ profile. The best agreement is obtained assuming for the ion heat transport $\chi_{i, s}=1.1$ for peaked $R / L_{T_{i, c r i t}}$ with nominal value of 3.2. This value of $\chi_{i, s}$ is lower than that yielded by GYRO by a factor of 2 and the stiffness of the ITG off-diagonal term must also be 2 times lower than the GYRO value to yield good electron results. This is a general observation in our modelling that the ratio $\chi_{i, s} / \chi_{i e, s}$ must be kept the same to obtained acceptable results for the electron channel. This is in agreement with the fact that these heat flux contributions are driven by the same turbulence.

It should be emphasised that the different phase lag of all the modulated ion and electron heat sources contribute to the central phase delay of the $T_{e}$ modulation and that the best agreement between simulations and experiment is obtained with the time constants and profiles yielded by the ICRH power deposition calculations. The profile of the phase delay in the outer part of the plasma seems to require a small amount of off-axis modulated electron heating power which is compatible with mode conversion in the ${ }^{3} \mathrm{He}$ scheme.

Finally, the modelling study indicates that in these discharges, in contrast to the situation for the ion channel, for the electrons the classical heat pulse analysis deduced directly from the experimental data, $\chi_{e}^{H P}$, is not valid due to the lack of a reliably well-defined source-free radial region.

\section{Conclusion}

The main goal of the experiment, which consisted in investigating ion heat transport with power modulation, has been achieved. The validity of the $T_{i}$ modulation approach for transport studies has been demonstrated, $\chi_{i}^{H P}$, as well as values of stiffness and threshold could be deduced.

The critical gradient model applied to ion heat transport seems to be a good hypothesis to interpret the data of our modulation experiments. This has been confirmed by non-linear gyro-kinetic calculations based on the experimental data taken at mid-radius. They yield, at this radial position, threshold $R / L_{T_{i, c r i t}}=3.2$ and stiffness $\chi_{i, s}=2.2$, as well as an almost linear 
increase of the ion heat flux above the threshold, $\alpha_{i} \approx 0.5$. The interpretation of the ion data with the critical gradient model provides results which of course depend on the assumption. Whereas flat profiles of $\chi_{i, s}$ and $R / L_{T_{i, c r i t}}$ yield the poorest agreement with the experiment, peaked $R / L_{T_{i, c r i t}}$ or hollow $\chi_{i, s}$ improve the match in different ways, but none of them can be clearly favoured compared to the other. Assuming for the threshold $R / L_{T_{i, \text { crit }}}=3.2$ yields good agreement with the experiment and corresponds to the GYRO result. The stiffness values, at mid radius, yielding an acceptable match with the experiment are 1.1 for flat $\chi_{i, s}$ and 2.2 for hollow $\chi_{i, s}$. This is respectively 2 times lower than and close to the GYRO results. These stiffness values correspond to the lower boundary of the range reported in [17], with which they are in agreement considering the experimental conditions and in particular the finite toroidal rotation. In addition, our non-linear gyro-kinetic calculations indicate that the presence of ${ }^{3} \mathrm{He}$ and the situation $R / L_{T_{e}}>R / L_{T_{i}}$ tend to reduce the stiffness.

This value of the ion stiffness could be measured correctly by the modulation method in our range of modulation frequency because it is not too high. A higher stiffness would require higher modulation frequencies than used here to avoid the problematic influence of the edge. Using high modulation frequencies is expected to severely reduce the $T_{i}$ modulation amplitude, and therefore the signal-to-noise ratio, due to the collisional time constant, in addition to the usual amplitude dependence on frequency which is intrinsic to the method. However, it should be underlined that a scan in $f_{\text {mod }}$ covering frequencies above $20 \mathrm{~Hz}$ would also yield precious information for a better assessment of the collisional time constants of the RFaccelerated ions.

The ${ }^{3} \mathrm{He}$ ICRH scheme in deuterium plasmas works well for ion modulation and is, to our knowledge, the best possibility for such experiments, despite the accompanying electron heating. The ${ }^{3} \mathrm{He}$ concentration was perhaps somewhat too high in some of our cases, leading to slow time variation of the heating and/or transport properties. Therefore, for future experiments, it seems desirable to keep the concentration at the lower boundary of the range predicted for good ion heating. In our experiments, the ${ }^{3} \mathrm{He}$ concentration profile could only be measured in one discharge. The availability of more profiles of the ${ }^{3} \mathrm{He}$ concentration would significantly improve the ICRH power deposition calculations.

The situation in the electron channel is complex because it involves up to three heating sources with different time scales and radial profiles. In addition, in these discharges with comparable levels of ion and electron heating power, electron heat transport is dominated by the off- 
diagonal term caused by the ITG instability, driven by $R / L_{T_{i}}$. In comparison, the diffusion due to the TEM instability is weak in the plasma core. Due to this complexity, our results do not yield an absolutely definitive answer on electron heat transport and ICRH electron heating under such conditions, but the following conclusions can be drawn. We consider the non-monotonic shape of the phase profile as an important experimental feature which must be, at least qualitatively, reproduced by the modelling. Modelling with CGM assuming pure diffusion partially reproduces this aspect only if off-axis electron heating is added and low stiffness assumed. However, the modelled modulation amplitude at low frequency is much too low. Taking the off-diagonal term into account yields much better results, including the non-monotonic shape of the phase profile and the correct amplitude at the two frequencies. Solely, the phase in the outer plasma region is clearly too steep. This can be significantly improved with off-axis modulated heating power, in the range of $10 \%$ of the total ICRH electron heating power. Therefore, our results strongly support the off-diagonal ITG contribution to electron heat transport predicted by GYRO, whereas the existence of a small off-axis mode conversion contribution to electron heating power seems required. It is important to emphasise that good modelling results are obtained with values compatible with those yielded by the gyro-kinetic transport results from GYRO (e.g. $R / L_{T_{i, c r i t}}=3.2$ and $\alpha_{i}=0.5$ ) and require the ICRH power deposition as calculated by SELFO.

Using $T_{e}$ modulation provided by the ${ }^{3} \mathrm{He}$ ICRH mode conversion scheme, electron heat transport has been previously investigated in JET discharges with various fractions of electron and ion heating. The interpretation of the electron heat transport with CGM assuming pure diffusion (diagonal term only) required an increase of both residual transport $\chi_{e, 0}$ and electron stiffness $\chi_{e, s}$, with increasing ion heating, [40]. The non-diagonal ITG contribution to electron heat transport revealed by our study agrees with the high value of $\chi_{e, 0}$, as underlined by the authors in Ref. [40] page 1159. The increase of $\chi_{e, s}$ might also be due to it, caused by a concomitant (small) modulation of $T_{i}$, or of $\chi_{i e}$, which could not be evidenced with the available sensitivity of the CXRS diagnostic. Clarifying this question in future experiments would be highly desirable.

The mode conversion power deposition depends on the ${ }^{3} \mathrm{He}$ concentration which might have varied somewhat in our study. Its contribution is probably small but it cannot be ignored, as shown by its strong influence on the phase profile of the $T_{e}$ modulation, as indicated by our modelling results. This effect could not be assessed directly from the experimental data in 
our study. This could be done in future experiments using a high modulation frequency for which the $T_{i}$ and $P_{e, \text { coll }}$ contributions to $T_{e}$ modulation would be completely smoothed out due to their long time constants. The remaining contributions to the $T_{e}$ modulation would be the central direct heating and the off-axis contribution which could then be identified in the amplitude and phase profiles of the $T_{e}$ modulation. Data at such a high frequency are not available in the existing discharges, but would be a useful extension in future experiments.

Future experiments, similar to those presented here, could also take advantage of further improvements made to the $T_{i}$ measurement in the recent years and from the edge $T_{i}$ measurement which was not available for our study.

The ITG-driven electron heat transport plays a crucial role in present, as it also will in future devices, but has been rarely investigated because the experimental possibilities are very limited. JET offers, at present, a worldwide unique opportunity to investigate this important aspect of transport through the ICRH ${ }^{3} \mathrm{He}$ minority scheme. Due to the constraints of the future JET experimental programme, $T_{i}$ modulation experiments will be possible earliest from 2012 onwards. The importance of the topic and the results presented here strongly support the wish for future studies dedicated to ion transport and ITG-driven electron heat transport, along the lines suggested above.

\section{Acknowledgment}

The authors are very grateful to J. Candy and R.E. Waltz for providing the gyro-kinetic code GYRO. Simulations were performed at the parallel server Power-6 (Vip) of the IPP-MPG Rechenzentrum in Garching bei München, Germany. This work, supported by the European Communities under the contract of Association EURATOM-IPP, was carried out within the framework of the EFDA. The views and opinions expressed herein do not necessarily reflect those of the European Commission.

\section{References}

[1] DIMITS, A. et al., Phys. Plasmas 7 (2000) 969.

[2] PEeters, A. G. et al., Physics of Plasmas 12 (2005) 022505.

[3] GREENWALD, M. et al., Nucl. Fusion 37 (1997) 793.

[4] SUTTROP, W. et al., Plasma Physics and Controlled Fusion 39 (1997) 2051.

[5] GOHIL, P. et al., Nucl. Fusion 38 (1998) 425.

[6] HORTON, L. D. et al., Plasma Physics and Controlled Fusion 41 (1999) B329.

[7] RYTER, F. et al., Plasma Physics and Controlled Fusion 43 (2001) A323. 
[8] URANO, H. et al., Nucl. Fusion 42 (2002) 76 .

[9] PEETERS, A. G. et al., Nuclear Fusion 42 (2002) 1376.

[10] TARDINI, G. et al., Nuclear Fusion 42 (2002) 258.

[11] RYTER, F. et al., Plasma Physics and Controlled Fusion 48 (2006) B453.

[12] RYTER, F. et al., Nuclear Fusion 43 (2003) 1396.

[13] RYTER, F. et al., Physical Review Letters 95 (2005) 085001.

[14] DEBOO, J. C. et al., Nuclear Fusion 45 (2005) 494.

[15] CAMENEN, Y. et al., Plasma Physics and Controlled Fusion 47 (2005) 1971.

[16] RYTER, F. et al., Simultaneous analysis of ion and electron heat transport by power modulation in JET, in Proc. of the 22nd IAEA Conference Fusion Energy (CD-Rom), Geneva, Switzerland, October 2008, volume IAEA-CSP-25/CD, pages IAEA-CN-165/EX/P5-19 and http://www-naweb.iaea.org/napc/physics/FEC/FEC2008/html/index.htm_http, Vienna, 2008, IAEA.

[17] MANTICA, P. et al., Phys. Rev. Lett. 102 (2009) 175002.

[18] LOPES CARDOZO, N. J., Plasma Phys. Controlled Fusion 37 (1995) 799.

[19] MANTICA, P. et al., Nucl. Fusion 36 (1996) 1317.

[20] RYTER, F. et al., Nuclear Fusion 41 (2001) 537.

[21] MANTICA, P. et al., Comptes Rendus Physique 7 (2006) 634.

[22] CASATI, A. et al., Phys. Plasmas 14 (2007) 092303.

[23] VAN EESTER, D. et al., Plasma Phys. Controlled Fusion 51 (2009) 044007.

[24] ERIKSSON, L.-G. et al., Nucl. Fusion 33 (1993) 1037.

[25] HEDIN, J. et al., Nuclear Fusion 42 (2002) 527.

[26] MANTSINEN, M. et al., Nucl. Fusion 44 (2004) 33.

[27] BRAMBILlA, M., Plasma Physics and Controlled Fusion 41 (1999) 1.

[28] NEGUS, C. R. et al., Rev. Sci. Instrum. 77 (2006) 10 F102.

[29] GIROUD, C. et al., Rev. Sci. Instrum. 79 (2008) 10F525.

[30] TUBBING, B. J. D. et al., Nucl. Fusion 27 (1987) 1843.

[31] RYTER, F. et al., Plasma Physics and Controlled Fusion 52 (2010) 124043.

[32] JACCHIA, A. et al., Phys. Fluids B 3 (1991) 3033.

[33] LEUTERER, F. et al., Nuclear Fusion 43 (2003) 744.

[34] PANKIN, A. et al., Computer Phys. Comm. 159 (2004) 157.

[35] WALTZ, R. E. et al., Phys. Plasmas 4 (1997) 2482.

[36] PEREVERZEV, G. V. et al., IPP report 5/98 (2002).

[37] IMBEAUX, F. et al., Plasma Physics and Controlled Fusion 43 (2001) 1503.

[38] GARBET, X. et al., Plasma Physics and Controlled Fusion 46 (2004) 1351, Addendum in Plasma Physics and Controlled Fusion 47, 6, pp. 957-958 (2005).

[39] CANDY, J. et al., Journal of Computational Physics 186 (2003) 545.

[40] MANTICA, P. et al., Fusion Science and Technology 53 (2008) 1152.

[41] JACCHIA, A. et al., Phys. Plasmas 2 (1995) 4589.

[42] STROTH, U. et al., Plasma Phys. Controlled Fusion 38 (1996) 611, Corrigendum in Plasma Phys. Contr. Fus., 381087 (1996). 